\title{
The $\mathcal{N}=4$ higher spin algebra for generic $\mu$ parameter
}

\author{
Changhyun Ahn ${ }^{a}$ and Man Hea Kim ${ }^{b}$ \\ ${ }^{a}$ Department of Physics, Kyungpook National University, \\ Taegu 41566, Korea \\ ${ }^{b}$ Asia Pacific Center for Theoretical Physics, \\ Pohang 37673, Korea \\ E-mail: ahn@knu.ac.kr, manhea.kim@apctp.org
}

ABSTRACT: The $\mathcal{N}=4$ higher spin generators for general superspin $s$ in terms of oscillators in the matrix generalization of $A d S_{3}$ Vasiliev higher spin theory at nonzero $\mu$ (which is equivalent to the 't Hooft-like coupling constant $\lambda$ ) were found previously. In this paper, by computing the (anti)commutators between these $\mathcal{N}=4$ higher spin generators for low spins $s_{1}$ and $s_{2}\left(s_{1}+s_{2} \leq 11\right)$ explicitly, we determine the complete $\mathcal{N}=4$ higher spin algebra for generic $\mu$. The three kinds of structure constants contain the linear combination of two different generalized hypergeometric functions. These structure constants remain the same under the transformation $\mu \leftrightarrow(1-\mu)$ up to signs. We have checked that the above $\mathcal{N}=4$ higher spin algebra contains the $\mathcal{N}=2$ higher spin algebra, as a subalgebra, found by Fradkin and Linetsky some time ago.

KEywords: AdS-CFT Correspondence, Conformal and W Symmetry, Higher Spin Gravity, Higher Spin Symmetry

ArXiv EPRINT: 2009.04852 


\section{Contents}

1 Introduction 1

2 Review 2

2.1 The fundamental relations 2

2.2 The $\mathcal{N}=4$ generators 3

2.3 The $s$-th $\mathcal{N}=4$ higher spin generators 4

2.4 The goal of this paper 6

3 Direct calculation of (anti)commutators between the oscillators $\quad 7$

3.1 The (anti)commutators between the oscillators with vanishing $\mu \quad 8$

3.2 The (anti)commutators between the oscillators with nonzero $\mu \quad 11$

4 The $\mathcal{N}=4$ higher spin algebra $\operatorname{shs}_{2}[\mu] \quad 15$

$\begin{array}{lll}4.1 & \text { Mode dependent structure constants } & 15\end{array}$

4.2 The (anti)commutators between the $\mathcal{N}=4$ higher spin generators with $\begin{array}{ll}\text { nonzero } \mu & 19\end{array}$

5 Conclusions and outlook $\quad 22$

$\begin{array}{ll}\text { A Structure constants for vanishing } \mu & 23\end{array}$

B The (anti)commutators between the oscillators with vanishing $\mu$ in Odake basis 24

C $\mu$-dependence in the (anti)commutators of the oscillators up to a total spin $11\left(h_{1}+h_{2} \leq 11\right) \quad 27$

C.1 The case-two with one bosonic and one fermionic oscillators 27

$\begin{array}{lll}\text { C.1.1 No operator } K \text { dependence in the oscillators } & 27\end{array}$

C.1.2 Operator $K$ dependence in the oscillators 30

C.2 The case-three with two fermionic oscillators 34

C.2.1 No operator $K$ dependence in the oscillators 34

$\begin{array}{lll}\text { C.2.2 } & \text { Operator } K \text { dependence in the oscillators } & 37\end{array}$

D The (anti)commutators between the oscillators with nonzero $\mu$ in terms of generalized hypergeometric functions 42

E The remaining (anti)commutators between the $\mathcal{N}=4$ higher spin generators

E.1 The four (anti)commutators with common $\Phi_{\frac{1}{2}, \rho}^{\left(s_{1}\right),}$

E.2 The three (anti)commutators with common $\Phi_{1, m}^{\left(s_{1}\right), i j} \quad 48$

E.3 The two (anti)commutators with common $\tilde{\Phi}_{\frac{3}{2}, \rho}^{\left(s_{1}\right), i} \quad 49$

E.4 The final commutator 50 
F The $\mathcal{N}=\mathbf{2}$ higher spin algebra $\operatorname{shs}[\lambda] \quad 50$

F.1 Review 50

F.2 How to obtain the $\mathcal{N}=2$ higher spin algebra $\operatorname{sh} s[\lambda]$ from $\mathcal{N}=4$ higher spin algebra $s h s_{2}[\lambda] \quad 52$

F.2.1 The first commutator of eq. (F.1) 54

F.2.2 Other (anti)commutators of eq. (F.1) 54

F.3 The (anti)commutators of oscillators by using the structure constants in $s h s[\mu] 55$

F.3.1 The structure constants in the $\operatorname{shs}[\mu]$ by using the generalized hypergeometric functions 56

F.3.2 The relations between the structure constants in the $s h s_{2}[\mu]$ and those in the $\operatorname{sh} s[\mu]$

G The (anti)commutators of oscillators by using the structure constants in

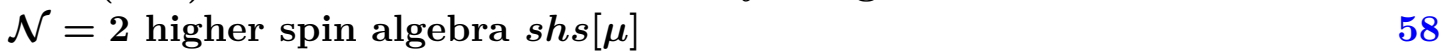

\section{Introduction}

It is known that there exists a large $\mathcal{N}=4$ holography [1] which connects the matrix generalization of the Vasiliev higher spin theory $[2,3]$ on $A d S_{3}$ with the two dimensional minimal model conformal field theories having the large $\mathcal{N}=4$ superconformal symmetry. One of the connections can be seen from the fact that the oscillator deformation parameter in the $A d S_{3}$ bulk theory corresponds to the free parameter of the large $\mathcal{N}=4$ superconformal algebra. The former is related to the mass of the scalar field while the latter is given by a particular combination of $N$ and a level $k$ in the $\mathcal{N}=4$ unitary coset model. There are seven spin 1 fields and eight fields of spin $s=\frac{3}{2}, 2, \frac{5}{2}, 3, \cdots$ in the (matrix valued) Vasiliev higher spin theory. They correspond to the $\mathcal{N}=4$ higher spin multiplets in the boundary theory which generate the nonlinear $\mathcal{W}_{\infty}^{\mathcal{N}=4}[\lambda]$ algebra where $\lambda$ is the 't Hooft-like coupling constant (above free parameter). By imposing the wedge condition and taking the infinity limit of central charge (or infinity limit of $N$ ) into the $\mathcal{W}_{\infty}^{\mathcal{N}}=4[\lambda]$ algebra, we are left with the linear higher spin algebra $s h s_{2}[\mu]$ generated by above higher spin fields in the $A d S_{3}$ bulk theory, via the large $\mathcal{N}=4$ holography. See also relevant works in [4-8] for the emergence of higher spin symmetry.

So far, the nonlinear $\mathcal{W}_{\infty}^{\mathcal{N}=4}[\lambda]$ algebra in the $\mathcal{N}=4$ unitary coset model is known, for generic $\lambda$, from both the OPE between the first $\mathcal{N}=4$ higher spin multiplet and the OPE between the first and second $\mathcal{N}=4$ higher spin multiplets [9-11]. Beyond these OPEs, it is rather nontrivial to obtain more OPEs. See also relevant works in [12-19] for the large $\mathcal{N}=4$ holography. On the other hand, at the vanishing $\lambda$ after taking infinity limit of $N$, the linear $\mathcal{W}_{\infty}^{\mathcal{N}}=4$ [0] algebra is realized by $2 N$ free complex bosons and $2 N$ free complex fermions through bilinear construction $[12,20]$ and the (anti)commutators with explicit $\mathrm{SO}(4)$ symmetry between the $\mathcal{N}=4$ higher spin currents in component approach are completely determined for generic spins $s_{1}$ and $s_{2}$ [11]. See also the relevant works [21-24] for the construction of its bosonic subsector. In this case, the corresponding $\mathcal{N}=4$ higher spin algebra $s h s_{2}[0]$ with nondeformed parameter can be determined by restricting the 
mode indices of higher spin currents to the wedge indices. See also previous works [25-31] on $\mathcal{N}=2$ higher spin algebra shs $[\mu]$ some time ago.

In this paper, we would like to construct the $\mathcal{N}=4$ higher spin algebra $\operatorname{sh} s_{2}[\mu]$ for generic $\mu$ (or $\lambda$ ) parameter. All we have is the $\mathcal{N}=4$ higher spin generators for superspin $s$ in terms of the multiple products of oscillators in the matrix generalization of $A d S_{3}$ Vasiliev higher spin theory for nonzero $\mu$ [11]. We can compute the various (anti)commutators between these $\mathcal{N}=4$ higher spin generators for low spins $s_{1}$ and $s_{2}$ by using the fundamental (anti)commutator relations between the oscillators. We have considered the total spin $s=s_{1}+s_{2}$ which is less than or equal to 11 . Therefore, all the structure constants depend on $\lambda$ (or $\mu$ ) for these fixed $s_{1}$ and $s_{2}$ explicitly. We want to generalize these (anti)commutators in terms of closed analytic form for any arbitrary $s_{1}$ and $s_{2}$. We expect to have that the $\mathrm{SO}(4)$ index structure and mode dependent parts in the (anti)commutators for nonzero $\lambda$ (or $\mu$ ) in $s h s_{2}[\mu]$ remain the same as the ones for vanishing $\lambda$ in $s h s_{2}[0]$. The structure constants are function of $s_{1}, s_{2}$ and $\lambda$ as well as modes. The question is how we allocate these variables appropriately in the generalized hypergeometric functions in which there are four upper arguments and three lower arguments in addition to one variable.

In section 2, after introducing the fundamental (anti)commutator relations for the oscillators, the realizations for the $\mathcal{N}=4$ (higher spin) generators are given explicitly. The aim of this paper is sketched briefly.

In section 3, after introducing the various (anti)commutators for the oscillators with vanishing $\lambda$, they for nonzero $\lambda$ are determined by considering the several $s_{1}$ and $s_{2}$ values.

In section 4, based on the results of section 3, the three (or six) kinds of structure constants are obtained and the closed forms are presented in terms of generalized hypergeometric functions explicitly. Moreover, the final (anti)commutator relations for the $\mathcal{N}=4$ higher spin generators, $s h s_{2}[\mu]$, are determined completely.

In section 5, we present the summary of this paper.

In appendices, some various detailed results in sections 3,4 are given explicitly. In appendix $\mathrm{F}$, the $\mathcal{N}=2$ higher spin algebra $\operatorname{sh} s[\mu]$, as a subalgebra of $s h s_{2}[\mu]$, is obtained from the results of section 4. See also relevant works [32-44] on this direction.

See also recent works in [45-47]. The mathematica [48] is used.

\section{Review}

\subsection{The fundamental relations}

The generators of $\mathcal{N}=4$ higher spin algebra denoted by $s_{2} s_{2}[\mu]$ are given by the tensor product between the generators of $\mathcal{N}=2$ higher spin algebra denoted by $\operatorname{shs}[\mu]$ and the generators of $2 \times 2$ matrices. The generators of $\mathcal{N}=2$ higher spin algebra $\operatorname{shs}[\mu]$ can be constructed by the multiple products between the oscillators $\hat{y}_{\alpha}(\alpha=1,2)$ and the operator $K$ and they satisfy the following fundamental relations $[49-51]^{1}$

$$
\left[\hat{y}_{\alpha}, \hat{y}_{\beta}\right]_{\star}=2 \mathrm{i} \epsilon_{\alpha \beta}(1+\nu K), \quad\left\{K, \hat{y}_{\alpha}\right\}_{\star}=0, \quad K^{2}=1 .
$$

\footnotetext{
${ }^{1}$ We denote i by an imaginary number.
} 
Without a deformation $(\nu=0)$, the usual oscillator commutation relation in $\hat{y}_{\alpha}$ is satisfied in the first relation of (2.1). Here $\nu=2 \mu-1$. The $\epsilon_{12}=-\epsilon_{21}=1$ tensor is antisymmetric. The product between the oscillators and the operator $K$ is a Moyal product [52] by using $\mathrm{a} \star$ notation. For example, the (anti)commutators between the oscillators are given by ${ }^{2}$

$$
\left[\hat{y}_{\alpha}, \hat{y}_{\beta}\right\}_{\star}=\hat{y}_{\alpha} \star \hat{y}_{\beta} \pm \hat{y}_{\beta} \star \hat{y}_{\alpha} .
$$

The plus sign in (2.2) corresponds to the anticommutator while the minus sign corresponds to the commutator.

We introduce an underbrace notation with the number of oscillators as the completely symmetrized product between them $[35]^{3}$

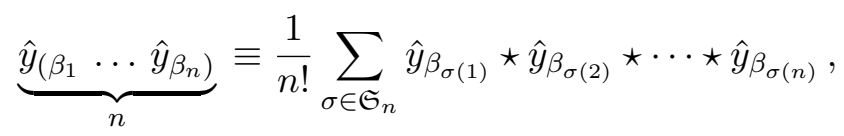

where $\mathfrak{S}_{n}$ in (2.3) stands for the group of permutations of $n$ elements.

The precise relation between the parameter $\nu$ (or $\mu$ ) in the $\mathcal{N}=4$ higher spin algebra and the parameter $\lambda$ in the $\mathcal{N}=4$ coset model in two dimensions is as follows [1]:

$$
\nu=2 \lambda-1, \quad \text { or } \quad \lambda=\mu=\frac{(\nu+1)}{2} .
$$

See also the relevant works in [52-54] for the matrix generalization of the $A d S_{3}$ Vasiliev higher spin theory.

From now on we will use the $\mu$ parameter rather than $\lambda$ parameter all the time.

\subsection{The $\mathcal{N}=4$ generators}

By using the $\mathrm{SO}(4)$ symmetry of the $\mathcal{N}=4$ superconformal algebra [55-57], we rearrange the $\mathrm{SO}(4)$ vector and adjoint indices in order to match them with those of Pauli matrices $\sigma^{i}(i=1,2,3)[1,58]$.

$$
\begin{aligned}
L_{m} & =\frac{1}{4} \underbrace{\hat{y}_{\left(1 \ldots \hat{y}_{1}\right.}}_{1+m} \underbrace{\hat{y}_{2} \ldots \hat{y}_{2)}}_{1-m} \otimes\left(\begin{array}{ll}
1 & 0 \\
0 & 1
\end{array}\right), \quad|m|<2, \\
G_{ \pm \frac{1}{2}}^{1} & =\frac{1}{2} e^{\mathrm{i} \frac{\pi}{4}} \hat{y}_{\frac{3}{2} \mp \frac{1}{2}} K \otimes \sigma^{1}, \quad G_{ \pm \frac{1}{2}}^{2}=-\frac{1}{2} e^{\mathrm{i} \frac{\pi}{4}} \hat{y}_{\frac{3}{2} \mp \frac{1}{2}} K \otimes \sigma^{2}, \\
G_{ \pm \frac{1}{2}}^{3} & =\frac{1}{2} e^{\mathrm{i} \frac{\pi}{4}} \hat{y}_{\frac{3}{2} \mp \frac{1}{2}} K \otimes \sigma^{3}, \quad G_{ \pm \frac{1}{2}}^{4}=\frac{\mathrm{i}}{2} e^{\mathrm{i} \frac{\pi}{4}} \hat{y}_{\frac{3}{2} \mp \frac{1}{2}} \otimes\left(\begin{array}{ll}
1 & 0 \\
0 & 1
\end{array}\right), \\
T_{0}^{12} & =\frac{1}{2} \otimes \sigma^{3}, \quad T_{0}^{13}=\frac{1}{2} \otimes \sigma^{2}, \quad T_{0}^{23}=\frac{1}{2} \otimes \sigma^{1}, \\
T_{0}^{14} & =-\frac{1}{2} K \otimes \sigma^{1}, \quad T_{0}^{24}=\frac{1}{2} K \otimes \sigma^{2}, \quad T_{0}^{34}=-\frac{1}{2} K \otimes \sigma^{3}, \quad\left(T^{i j}=-T^{j i}\right) .
\end{aligned}
$$

The $\epsilon^{123}=1$ tensor is antisymmetric. The $\mathcal{N}=4$ wedge algebra generated by nine bosonic $\left(L_{m}, T_{0}^{i j}, T_{0}^{i 4}\right)$ and eight fermionic $\left(G_{ \pm \frac{1}{2}}^{i}, G_{ \pm \frac{1}{2}}^{4}\right)$ generators in (2.5) can be obtained and

\footnotetext{
${ }^{2}$ For simplicity, we will not introduce a $\star$ notation explicitly between the oscillators and the operator $K$.

${ }^{3} \operatorname{In}[11]$, there is no prefactor $\frac{1}{n !}$.
} 
denoted by the global exceptional superalgebra $D(2,1 \mid \alpha)$ with $\alpha \equiv \frac{(1+\nu)}{(1-\nu)}[1]$. We can obtain this $\mathcal{N}=4$ wedge algebra by restricting to the wedge mode from the standard (anti)commutators of the large $\mathcal{N}=4$ superconformal algebra after performing the large $(N, k)$ 't Hooft limit. ${ }^{4}$

\subsection{The $s$-th $\mathcal{N}=4$ higher spin generators}

In [11], the $s$-th $\mathcal{N}=4$ higher spin generators written in terms of the component field in $\mathcal{N}=4$ superspace are described by the oscillators

$$
\begin{aligned}
& \Phi_{0, m}^{(s)}=\underbrace{\hat{y}_{(1} \ldots \hat{y}_{1}}_{s-1+m} \underbrace{\hat{y}_{2} \ldots \hat{y}_{2)}}_{s-1-m}(K(2 s-1)+\nu) \otimes\left(\begin{array}{ll}
1 & 0 \\
0 & 1
\end{array}\right), \quad-(s-1) \leq m \leq(s-1), \\
& \Phi_{\frac{1}{2}, \rho}^{(s), 1}=-(2 s-1) e^{\mathrm{i} \frac{\pi}{4}} \underbrace{\hat{y}_{(1} \ldots \hat{y}_{1}}_{s-\frac{1}{2}+\rho} \underbrace{\hat{y}_{2} \ldots \hat{y}_{2)}}_{s-\frac{1}{2}-\rho} \otimes \sigma^{1}, \quad-\left(s-\frac{1}{2}\right) \leq \rho \leq\left(s-\frac{1}{2}\right), \\
& \Phi_{\frac{1}{2}, \rho}^{(s), 2}=(2 s-1) e^{\mathrm{i} \frac{\pi}{4}} \underbrace{\hat{y}_{(1 \ldots \hat{y}}}_{s-\frac{1}{2}+\rho} \underbrace{\hat{y}_{2} \ldots \hat{y}_{2)}}_{s-\frac{1}{2}-\rho} \otimes \sigma^{2}, \quad-\left(s-\frac{1}{2}\right) \leq \rho \leq\left(s-\frac{1}{2}\right), \\
& \Phi_{\frac{1}{2}, \rho}^{(s), 3}=-(2 s-1) e^{\mathrm{i} \frac{\pi}{4}} \underbrace{\hat{y}_{\left(1 \ldots \hat{y}_{1}\right.}}_{s-\frac{1}{2}+\rho} \underbrace{\hat{y}_{2} \ldots \hat{y}_{2)}}_{s-\frac{1}{2}-\rho} \otimes \sigma^{3}, \quad-\left(s-\frac{1}{2}\right) \leq \rho \leq\left(s-\frac{1}{2}\right), \\
& \Phi_{\frac{1}{2}, \rho}^{(s), 4}=-\mathrm{i}(2 s-1) e^{\mathrm{i} \frac{\pi}{4}} \underbrace{\hat{y}_{(1} \ldots \hat{y}_{1}}_{s-\frac{1}{2}+\rho} \underbrace{\left.\hat{y}_{2} \ldots \hat{y}_{2}\right)}_{s-\frac{1}{2}-\rho} K \otimes\left(\begin{array}{ll}
1 & 0 \\
0 & 1
\end{array}\right), \quad-\left(s-\frac{1}{2}\right) \leq \rho \leq\left(s-\frac{1}{2}\right), \\
& \Phi_{1, m}^{(s), 12}=-(2 s-1) \underbrace{\hat{y}_{(1} \ldots \hat{y}_{1}}_{s+m} \underbrace{\left.\hat{y}_{2} \ldots \hat{y}_{2}\right)}_{s-m} \otimes \sigma^{3}, \quad-s \leq m \leq s, \\
& \Phi_{1, m}^{(s), 13}=-(2 s-1) \underbrace{\hat{y}_{(1} \ldots \hat{y}_{1}}_{s+m} \underbrace{\left.\hat{y}_{2} \ldots \hat{y}_{2}\right)}_{s-m} \otimes \sigma^{2}, \quad-s \leq m \leq s, \\
& \Phi_{1, m}^{(s), 23}=-(2 s-1) \underbrace{\hat{y}_{(1} \ldots \hat{y}_{1}}_{s+m} \underbrace{\hat{y}_{2} \ldots \hat{y}_{2)}}_{s-m} \otimes \sigma^{1}, \quad-s \leq m \leq s, \\
& \Phi_{1, m}^{(s), 14}=(2 s-1) \underbrace{\hat{y}_{\left(1 \ldots \hat{y}_{1}\right.}}_{s+m} \underbrace{\hat{y}_{2} \ldots \hat{y}_{2)}}_{s-m} K \otimes \sigma^{1}, \quad-s \leq m \leq s, \\
& \Phi_{1, m}^{(s), 24}=-(2 s-1) \underbrace{\hat{y}_{\left(1 \ldots \hat{y}_{1}\right.}}_{s+m} \underbrace{\hat{y}_{2} \ldots \hat{y}_{2)}}_{s-m} K \otimes \sigma^{2}, \quad-s \leq m \leq s, \\
& \Phi_{1, m}^{(s), 34}=(2 s-1) \underbrace{\hat{y}_{(1} \ldots \hat{y}_{1}}_{s+m} \underbrace{\hat{y}_{2} \ldots \hat{y}_{2}}_{s-m} K \otimes \sigma^{3}, \quad-s \leq m \leq s, \quad\left(\Phi_{1, m}^{(s), i j}=-\Phi_{1, m}^{(s), j i}\right)
\end{aligned}
$$

\footnotetext{
${ }^{4}$ By identifying $A^{ \pm 3}=\frac{1}{2}\left(T^{12} \mp T^{34}\right)$ and $G^{ \pm}=\frac{1}{\sqrt{2}}\left(G^{1} \mp \mathrm{i} G^{2}\right)$ with the corresponding generators of $\mathcal{N}=2$ superconformal algebra where the $\mathcal{U}(1)$ spin- 1 current is given by $J=2\left(\gamma A^{+3}+(1-\gamma) A^{-3}\right)$ with $\gamma \equiv \frac{\alpha}{(1+\alpha)}$, the $\mathcal{N}=2$ wedge algebra is generated by four bosonic $\left(L_{ \pm 1}, L_{0}, J_{0}\right)$ and four fermionic $G_{ \pm \frac{1}{2}}^{ \pm}$ generators [1]. We thank the referee for pointing this embedding and also the embedding of the $\mathcal{N}=2$ higher spin algebra $\operatorname{shs}[\mu]$ inside the $\mathcal{N}=4$ higher spin algebra $\operatorname{sh} s_{2}[\mu]$ out.
} 


$$
\begin{aligned}
& \tilde{\Phi}_{\frac{3}{2}, \rho}^{(s), 1}=\mathrm{i}(2 s-1) e^{\mathrm{i} \frac{\pi}{4}} \underbrace{\hat{y}_{(1} \ldots \hat{y}_{1}}_{s+\frac{1}{2}+\rho} \underbrace{\hat{y}_{2} \ldots \hat{y}_{2)}}_{s+\frac{1}{2}-\rho} K \otimes \sigma^{1}, \quad-\left(s+\frac{1}{2}\right) \leq \rho \leq\left(s+\frac{1}{2}\right), \\
& \tilde{\Phi}_{\frac{3}{2}, \rho}^{(s), 2}=-\mathrm{i}(2 s-1) e^{\mathrm{i} \frac{\pi}{4}} \underbrace{\hat{y}_{(1} \ldots \hat{y}_{1}}_{s+\frac{1}{2}+\rho} \underbrace{\left.\hat{y}_{2} \ldots \hat{y}_{2}\right)}_{s+\frac{1}{2}-\rho} K \otimes \sigma^{2}, \quad-\left(s+\frac{1}{2}\right) \leq \rho \leq\left(s+\frac{1}{2}\right), \\
& \tilde{\Phi}_{\frac{3}{2}, \rho}^{(s), 3}=\mathrm{i}(2 s-1) e^{\mathrm{i} \frac{\pi}{4}} \underbrace{\hat{y}_{(1} \ldots \hat{y}_{1}}_{s+\frac{1}{2}+\rho} \underbrace{\hat{y}_{2} \ldots \hat{y}_{2)}}_{s+\frac{1}{2}-\rho} K \otimes \sigma^{3}, \quad-\left(s+\frac{1}{2}\right) \leq \rho \leq\left(s+\frac{1}{2}\right), \\
& \tilde{\Phi}_{\frac{3}{2}, \rho}^{(s), 4}=-(2 s-1) e^{\mathrm{i} \frac{\pi}{4}} \underbrace{\hat{y}_{(1} \ldots \hat{y}_{1}}_{s+\frac{1}{2}+\rho} \underbrace{\left.\hat{y}_{2} \ldots \hat{y}_{2}\right)}_{s+\frac{1}{2}-\rho} \otimes\left(\begin{array}{ll}
1 & 0 \\
0 & 1
\end{array}\right), \quad-\left(s+\frac{1}{2}\right) \leq \rho \leq\left(s+\frac{1}{2}\right), \\
& \tilde{\Phi}_{2, m}^{(s)}=-(2 s-1) \underbrace{\hat{y}_{(1} \ldots \hat{y}_{1}}_{s+1+m} \underbrace{\hat{y}_{2} \ldots \hat{y}_{2)}}_{s+1-m} \otimes\left(\begin{array}{ll}
1 & 0 \\
0 & 1
\end{array}\right), \quad-(s+1) \leq m \leq(s+1) .
\end{aligned}
$$

The spin of each operator is given by the half of the number of oscillators plus one. We can check that the total number of higher spin generators is given by $16(1+2 s)$. The difference between the number of $\hat{y}_{1}$ and the number of $\hat{y}_{2}$ is two times of each mode. Note that if we rewrite the above higher spin generators by ignoring the symmetric combinations and moving all the $\hat{y}_{1}$ to the left, then there exist various types of oscillators having different lengths in their expressions. ${ }^{5}$

Recall that the large $\mathcal{N}=4$ superconformal algebra contains two $s u(2)$ algebra and the higher spin generators from the $D(2,1 \mid \alpha)$ multiplets [1]

$$
\begin{array}{rlll} 
& s:(\mathbf{1}, \mathbf{1}) & \longrightarrow & \Phi_{0, m}^{(s)} \\
& s+\frac{1}{2}:(\mathbf{2}, \mathbf{2}) & \longrightarrow & \Phi_{\frac{1}{2}, \rho}^{(s), i} \\
R^{(s)}: & s+1:(\mathbf{3}, \mathbf{1}) \oplus(\mathbf{1}, \mathbf{3}) & \longrightarrow & \Phi_{1, m}^{(s), i j} \\
& s+\frac{3}{2}:(\mathbf{2}, \mathbf{2}) & \longrightarrow & \tilde{\Phi}_{\frac{3}{2},(s), i}^{(s)} \\
& s+2:(\mathbf{1}, \mathbf{1}) & \longrightarrow & \tilde{\Phi}_{2, m}^{(s)}
\end{array}
$$

generate the $\mathcal{N}=4$ higher spin algebra $s s_{2}[\mu]$ and two bold-face numbers in (2.8) denote the representations with respect to the two $s u(2)$ algebra and $s=1,2, \cdots$.

\footnotetext{
${ }^{5}$ For example, there are following five bosonic generators of spin 3 for $s=1$

$$
\begin{aligned}
\tilde{\Phi}_{2,-2}^{(1)} & =-\hat{y}_{2} \hat{y}_{2} \hat{y}_{2} \hat{y}_{2} \otimes I_{2 \times 2}, \quad \tilde{\Phi}_{2,-1}^{(1)}=\left(-\hat{y}_{1} \hat{y}_{2} \hat{y}_{2} \hat{y}_{2}+\mathrm{i} \nu \hat{y}_{2} \hat{y}_{2} K+3 \mathrm{i} \hat{y}_{2} \hat{y}_{2}\right) \otimes I_{2 \times 2}, \\
\tilde{\Phi}_{2,0}^{(1)} & =\left(-\hat{y}_{1} \hat{y}_{1} \hat{y}_{2} \hat{y}_{2}+4 \mathrm{i} \hat{y}_{1} \hat{y}_{2}+\frac{2}{3}\left(3+4 \nu K+\nu^{2}\right)\right) \otimes I_{2 \times 2}, \\
\tilde{\Phi}_{2,+1}^{(1)} & =\left(-\hat{y}_{1} \hat{y}_{1} \hat{y}_{1} \hat{y}_{2}+\mathrm{i} \nu \hat{y}_{1} \hat{y}_{1} K+3 \mathrm{i} \hat{y}_{1} \hat{y}_{1}\right) \otimes I_{2 \times 2}, \quad \tilde{\Phi}_{2,+2}^{(1)}=-\hat{y}_{1} \hat{y}_{1} \hat{y}_{1} \hat{y}_{1} \otimes I_{2 \times 2} .
\end{aligned}
$$

Among these in (2.7) we observe that by moving the oscillator $\hat{y}_{1}$ appearing in (2.6) to the left some of the generators have different kinds of oscillators in the sense that each term in the middle three generators in (2.7) has different number of oscillators.
} 


\subsection{The goal of this paper}

The question we have raised in [11] is how we obtain the $\mathcal{N}=4$ higher spin algebra $\operatorname{sh} s_{2}[\mu]$ for nonzero $\mu$ by using the explicit realization of (2.6) via direct calculation with the help of $(2.1)$.

Schematically the generator of spin $h$ with mode $m$ has the following product of oscillators (the analysis for the oscillators with the operator $K$ can be done)

$$
\Phi_{m}^{h} \propto \underbrace{\hat{y}_{(1} \ldots \hat{y}_{1}}_{h-1+m} \underbrace{\hat{y}_{2} \ldots \hat{y}_{2)}}_{h-1-m} .
$$

Then we can construct the (anti)commutator between them in (2.9) as follows:

$$
\begin{aligned}
{\left[\Phi_{m_{1}}^{h_{1}}, \Phi_{m_{2}}^{h_{2}}\right\} \propto } & \underbrace{\hat{y}_{(1} \ldots \hat{y}_{1}}_{h_{1}-1+m_{1}} \underbrace{\hat{y}_{2} \ldots \hat{y}_{2}}_{h_{1}-1-m_{1}} \underbrace{\hat{y}_{(1} \ldots \hat{y}_{1}}_{h_{2}-1+m_{2}} \underbrace{\hat{y}_{2} \ldots \hat{y}_{2)}}_{h_{2}-1-m_{2}} \\
& \pm \underbrace{\hat{y}_{(1} \ldots \hat{y}_{1}}_{h_{2}-1+m_{2}} \underbrace{\left.\hat{y}_{2} \ldots \hat{y}_{2}\right)}_{h_{2}-1-m_{2}} \underbrace{\hat{y}_{(1} \ldots \hat{y}_{1}}_{h_{1}-1+m_{1}} \underbrace{\left.\hat{y}_{2} \ldots \hat{y}_{2}\right)}_{h_{1}-1-m_{1}} \\
= & \sum_{h_{3}=1}^{h_{1}+h_{2}-1}[c_{h_{3}}\left(h_{1}, h_{2}, m_{1}, m_{2}\right) \underbrace{\hat{y}_{(1} \ldots \ldots \hat{y}_{1}}_{h_{3}-1+m_{1}+m_{2}} \underbrace{\left.\hat{y}_{2} \ldots \ldots \hat{y}_{2}\right)}_{h_{3}-1-m_{1}-m_{2}} \\
& \quad+d_{h_{3}}\left(h_{1}, h_{2}, m_{1}, m_{2}\right) \underbrace{\hat{y}_{(1} \ldots \hat{y}_{1}-1-m_{1}-m_{2}}_{h_{3}-1+m_{1}+m_{2}} \underbrace{\left.\hat{y}_{2} \ldots\right]}_{\left.h_{2} \ldots \ldots \hat{y}_{2}\right)} K]
\end{aligned}
$$

The first expression of (2.10) is simply two terms from the (anti)commutator for given spins and modes. The next expression is that we would like to move the oscillator $\hat{y}_{1}$ to the left while the operator $K$ should be moved to the right in order to classify all the possible oscillators systematically. Due to the fundamental relations (2.1), the former will produce the oscillator term whose number of oscillator is reduced by 2 at one step movement while the latter will produce additional sign change. In this process after all the oscillators $\hat{y}_{1}$ are moved to the left, the right hand side contains different kinds of oscillators including the operator $K$. This implies the summation over the dummy variable $h_{3}$ in the above. At the final stage, we express the oscillators appearing in the right hand side in terms of $\mathcal{N}=4$ higher spin generators. The final goal is to obtain the structure constants explicitly for given spins $h_{1,2}$ and modes $m_{1}, m_{2}$. We would like to express them in terms of generalized hypergeometric functions. 


\section{Direct calculation of (anti)commutators between the oscillators}

Let us start with the particular commutator of $\mathcal{N}=4$ higher spin algebra $s h s_{2}[0]$ with vanishing $\mu$. The last commutator of appendix (J.1) in [11] is described by ${ }^{6}$

$$
\begin{aligned}
& {\left[\tilde{\Phi}_{2, m}^{\left(h_{1}\right)}, \tilde{\Phi}_{2, n}^{\left(h_{2}\right)}\right]=-\sum_{h=0}^{\left[\frac{h_{1}+h_{2}}{2}\right]} \frac{\left(2 h_{1}-1\right)\left(2 h_{2}-1\right)}{8\left(h_{1}+h_{2}-2 h\right)+12}[} \\
& \quad \times\left(p_{\mathrm{F}, 2 h}^{h_{1}+2, h_{2}+2}-p_{\mathrm{B}, 2 h}^{h_{1}+2, h_{2}+2}\right) \Phi_{0, m+n}^{\left(h_{1}+h_{2}-2 h+2\right)}-\frac{2}{2\left(h_{1}+h_{2}-2 h\right)-1} \\
& \left.\quad \times\left(\left(h_{1}+h_{2}-2 h+1\right) p_{\mathrm{F}, 2 h}^{h_{1}+2, h_{2}+2}+\left(h_{1}+h_{2}-2 h+2\right) p_{\mathrm{B}, 2 h}^{h_{1}+2, h_{2}+2}\right) \tilde{\Phi}_{2, m+n}^{\left(h_{1}+h_{2}-2 h\right)}\right] .
\end{aligned}
$$

The mode dependent structure constants $p_{F}$ and $p_{B}$ are given by appendix A. In order to extract the commutator constructed from the oscillators in the left hand side, we should change the higher spin generators into the corresponding oscillators by using (2.6)

$$
\begin{aligned}
{\left[\tilde{\Phi}_{2, m}^{\left(h_{1}\right)}, \tilde{\Phi}_{2, n}^{\left(h_{2}\right)}\right]=} & \frac{\mathrm{i}^{h_{1}+1}\left(2 h_{1}-1\right)}{8} \frac{\mathrm{i}^{h_{2}+1}\left(2 h_{2}-1\right)}{8} \\
& \times[\underbrace{\hat{y}_{\left(1 \ldots \hat{y}_{1}\right.}}_{h_{1}+1+m} \underbrace{\hat{y}_{2} \ldots \hat{y}_{2)}}_{h_{1}+1-m}, \underbrace{\hat{y}_{(1} \ldots \hat{y}_{1}}_{h_{2}+1+n} \underbrace{\left.\hat{y}_{2} \ldots \hat{y}_{2}\right)}_{h_{2}+1-n}] \otimes\left(\begin{array}{ll}
1 & 0 \\
0 & 1
\end{array}\right) .
\end{aligned}
$$

The prefactors come from the different normalization in this paper compared to the one in [11]. Since the commutator of two tensor products is given by the tensor product between commutator of oscillators and the $2 \times 2$ matrix product $[A \otimes I, B \otimes I]=(A B-B A) \otimes I$ for any operators $A$ and $B$, we can ignore the $2 \times 2$ identity matrix in this particular case. Then, the commutator from two bosonic oscillators in (3.2) can be written as

$$
\begin{aligned}
& {[\underbrace{\hat{y}_{(1} \ldots \hat{y}_{1}}_{h_{1}+1+m} \underbrace{\left.\hat{y}_{2} \ldots \hat{y}_{2}\right)}_{h_{1}+1-m}, \underbrace{\hat{y}_{(1} \ldots \hat{y}_{1}}_{h_{2}+1+n} \underbrace{\left.\hat{y}_{2} \ldots \hat{y}_{2}\right)}_{h_{2}+1-n}] \otimes\left(\begin{array}{ll}
1 & 0 \\
0 & 1
\end{array}\right) } \\
= & \frac{64}{\mathrm{i}^{h_{1}+h_{2}+2}\left(2 h_{1}-1\right)\left(2 h_{2}-1\right)}\left[\tilde{\Phi}_{2, m}^{\left(h_{1}\right)}, \tilde{\Phi}_{2, n}^{\left(h_{2}\right)}\right] .
\end{aligned}
$$

In this way, we can rewrite the various (anti)commutators between the oscillators in terms of those between the $\mathcal{N}=4$ higher spin generators like as (3.3). We can classify all possible cases as follows:

- The case-one

We have a single commutator by constructing two bosonic oscillators.

- The case-two

We have two kinds of commutators by considering one bosonic and one fermionic oscillators. There are two cases for the bosonic oscillator depending on the presence of the operator $K$ according to (2.6).

\footnotetext{
${ }^{6}$ The notation $[x]$ is the greatest integer not exceeding $x$ : the integer part of $x$.
} 
- The case-three

We have two kinds of anticommutators by considering two fermionic oscillators. There are two cases for the fermionic oscillator depending on the presence of the operator $K$.

We will construct the above five kinds of (anti)commutators explicitly in this paper. Although there are other cases, but they will belong to these three cases, in principle. Once we determine these (anti)commutators for the oscillators, we can obtain the $\mathcal{N}=4$ higher spin algebra $s h s_{2}[\mu]$ by multiplying the appropriate coefficients appearing in (2.6), which depend on the numerical factors, spins and the deformation parameter $\nu$, together with $2 \times 2$ matrices.

\subsection{The (anti)commutators between the oscillators with vanishing $\mu$}

In this subsection, we restrict to focus on the previous work [11] which holds for the vanishing $\mu$. In next subsection, we will construct the (anti)commutators between the oscillators with nonvanishing $\mu .^{7}$

- The case-one with two bosonic oscillators. We return to the relation (3.3). After substituting the last expression of (2.6) into the right hand side, we can compute this particular commutator. We can use the result of previous work of [11]: the last commutator of appendix (J.1) which is the right hand side of (3.1). We substitute the oscillator realization for the higher spin generators with the condition $\mu=0$ (or $\nu=-1$ in (2.4)). By shifting to $h_{i} \rightarrow h_{i}-2$ and introducing a dummy variable as $h \rightarrow r-1$, and simplifying the right hand side, we eventually obtain the summation of various commutators in the right hand side

$$
\begin{aligned}
& {[\underbrace{\hat{y}_{(1} \ldots \hat{y}_{1}}_{h_{1}-1+m} \underbrace{\hat{y}_{2} \ldots \hat{y}_{2}}_{h_{1}-1-m}, \underbrace{\hat{y}_{(1} \ldots \hat{y}_{1}}_{h_{2}-1+n} \underbrace{\left.\hat{y}_{2} \ldots \hat{y}_{2}\right)}_{h_{2}-1-n}]=} \\
& -\mathrm{i} \sum_{r=1}^{\left[\frac{h_{1}+h_{2}-1}{2}\right]} 2(-1)^{r}[\left(p_{\mathrm{F}, 2(r-1)}^{h_{1}, h_{2}}(m, n)+p_{\mathrm{B}, 2(r-1)}^{h_{1}, h_{2}}(m, n)\right) \underbrace{\hat{y}_{(1} \ldots \ldots \ldots \hat{y}_{1}}_{h_{1}+h_{2}-2 r-1+m+n} \underbrace{\hat{y}_{2} \ldots \ldots \ldots \hat{y}_{2)}}_{h_{1}+h_{2}-2 r-1-m-n} \\
& -\left(p_{\mathrm{F}, 2(r-1)}^{h_{1}, h_{2}}(m, n)-p_{\mathrm{B}, 2(r-1)}^{h_{1}, h_{2}}(m, n)\right) \underbrace{\hat{y}_{(1} \ldots \ldots \ldots \hat{y}_{1}}_{h_{1}+h_{2}-2 r-1+m+n} \underbrace{\left.\hat{y}_{2} \ldots \ldots \ldots \hat{y}_{2}\right)}_{h_{1}+h_{2}-2 r-1-m-n} K] .
\end{aligned}
$$

We denote $m, n, \cdots$ by the integer mode. The spin of right hand side is given by $\left(h_{1}+\right.$ $\left.h_{2}-2 r\right)$ from the previous analysis by counting the number of oscillators. Also the dummy variable $r$ starts with 1 . The structure constants are given in appendix A. ${ }^{8}$

\footnotetext{
${ }^{7}$ In this paper, the $h_{1}, h_{2}$ and $r$ for the spins are natural numbers.

${ }^{8}$ We observe that for the bosonic oscillators having the operator $K$, the relevant commutator is given by $[\underbrace{\hat{y}_{(1} \ldots \hat{y}_{1}}_{h_{1}-1+m} \underbrace{\hat{y}_{2} \ldots \hat{y}_{2)}}_{h_{1}-1-m} K, \underbrace{\hat{y}_{(1} \ldots \hat{y}_{1}}_{h_{2}-1+n} \underbrace{\hat{y}_{2} \ldots \hat{y}_{2)}}_{h_{2}-1-n} K]$. We can move the first operator $K$ in the plus sign to the right and then this meets with the second operator $K$. This becomes 1 from (2.1). Similarly, the first operator $K$ in the minus sign to the right and this meets with the second operator $K$. This also gives 1 . Then this commutator is the same as the one in (3.4). For the case where one of the oscillators contains
} 
- The case-two with one bosonic and on fermionic oscillators. Let us consider that the two kinds of oscillators do not have the operator $K$ dependence. We can use the fourth relation of appendix (J.1) of [11]. Together with the shifts, $h_{2} \rightarrow h_{1}-2, h_{1} \rightarrow h_{2}$ and $h \rightarrow r-\frac{3}{2}$, we substitute the corresponding higher spin generators given in (2.6) into the right hand side. It turns out that

$$
\begin{aligned}
& {[\underbrace{\hat{y}_{(1} \ldots \hat{y}_{1}}_{h_{1}-1+m} \underbrace{\left.\hat{y}_{2} \ldots \hat{y}_{2}\right)}_{h_{1}-1-m}, \underbrace{\hat{y}_{(1} \ldots \hat{y}_{1}}_{h_{2}-\frac{1}{2}+\rho} \underbrace{\hat{y}_{2} \ldots \hat{y}_{2}}_{h_{2}-\frac{1}{2}-\rho}]=} \\
& -4 \sum_{r=1}^{\left.\frac{h_{1}+h_{2}-1}{2}\right]}(-1)^{r}[\mathrm{i}\left(q_{\mathrm{F}, 2(r-1)}^{h_{1}, h_{2}+\frac{1}{2}}(m, \rho)+q_{\mathrm{B}, 2(r-1)}^{h_{1}, h_{2}+\frac{1}{2}}(m, \rho)\right) \underbrace{\hat{y}_{(1} \ldots \ldots \ldots \hat{y}_{1}}_{h_{1}+h_{2}-2 r-\frac{1}{2}+m+\rho} \underbrace{\hat{y}_{2} \ldots \ldots \ldots \hat{y}_{2}}_{h_{1}+h_{2}-2 r-\frac{1}{2}-m-\rho} \\
& -\left(q_{\mathrm{F}, 2 r-3}^{h_{1}, h_{2}+\frac{1}{2}}(m, \rho)+q_{\mathrm{B}, 2 r-3}^{h_{1}, h_{2}+\frac{1}{2}}(m, \rho)\right) \underbrace{\hat{y}_{(1} \ldots \ldots \ldots \hat{y}_{1}}_{h_{1}+h_{2}-2 r+\frac{1}{2}+m+\rho} \underbrace{\left.\hat{y}_{2} \ldots \ldots \ldots \hat{y}_{2}\right)}_{h_{1}+h_{2}-2 r+\frac{1}{2}-m-\rho} K] .
\end{aligned}
$$

We denote $\rho, \omega, \cdots$ by the half-integer mode. The spins of right hand side of (3.5) can be read off and are given by $\left(h_{1}+h_{2}-2 r+\frac{1}{2}\right)$ and $\left(h_{1}+h_{2}-2 r+\frac{3}{2}\right)$ respectively. The structure constants are given in appendix A. ${ }^{9}$

When the operator $K$ appears in the bosonic oscillator, we can use the second equation of (6.12) of [11]. By shifting the dummy variable $h \rightarrow r-\frac{3}{2}$, we obtain the following result

$$
\begin{aligned}
& {[\underbrace{\hat{y}_{(1} \ldots \hat{y}_{1}}_{h_{1}-1+m} \underbrace{\left.\hat{y}_{2} \ldots \hat{y}_{2}\right)}_{h_{1}-1-m} K, \underbrace{\hat{y}_{(1} \ldots \hat{y}_{1}}_{h_{2}-\frac{1}{2}+\rho} \underbrace{\hat{y}_{2} \ldots \hat{y}_{2)}}_{h_{2}-\frac{1}{2}-\rho}]=} \\
& -4 \sum_{r=1}^{\left[\frac{h_{1}+h_{2}}{2}\right]}[(-1)^{r}\left(q_{\mathrm{F}, 2 r-3}^{h_{1}, h_{2}+\frac{1}{2}}(m, \rho)-q_{\mathrm{B}, 2 r-3}^{h_{1}, h_{2}+\frac{1}{2}}(m, \rho)\right) \underbrace{\hat{y}_{(1} \ldots \ldots \ldots \hat{y}_{1}}_{h_{1}+h_{2}-2 r+\frac{1}{2}+m+\rho} \underbrace{\left.\hat{y}_{2} \ldots \ldots \ldots \hat{y}_{2}\right)}_{h_{1}+h_{2}-2 r+\frac{1}{2}-m-\rho} K] \\
& +4 \mathrm{i} \sum_{r=1}^{\left.\frac{h_{1}+h_{2}-1}{2}\right]}[(-1)^{r}\left(q_{\mathrm{F}, 2(r-1)}^{h_{1}, h_{2}+\frac{1}{2}}(m, \rho)-q_{\mathrm{B}, 2(r-1)}^{h_{1}, h_{2}+\frac{1}{2}}(m, \rho)\right) \underbrace{\hat{y}_{(1} \ldots \ldots \ldots \hat{y}_{1}}_{h_{1}+h_{2}-2 r-\frac{1}{2}+m+\rho} \underbrace{\left.\hat{y}_{2} \ldots \ldots \ldots \ldots \hat{y}_{2}\right)}_{h_{1}+h_{2}-2 r-\frac{1}{2}-m-\rho}] \text {. }
\end{aligned}
$$

the operator $K$, we consider the relevant commutator $[\underbrace{\hat{y}_{(1 \ldots} \hat{y}_{1}}_{h_{1}-1+m} \underbrace{\hat{y}_{2} \ldots \hat{y}_{2)}}_{h_{1}-1-m}, \underbrace{\hat{y}_{(1} \ldots \hat{y}_{1}}_{h_{2}-1+n} \underbrace{\hat{y}_{2} \ldots \hat{y}_{2)} K}_{h_{2}-1-n} K K$ where the additional operator $K$ is multiplied to the right. We can easily check that this commutator is the same as the one in (3.4) by moving the operator $K$ properly. Therefore, when one of the oscillators contains the operator $K$, the commutator can be obtained by multiplying the operator $K$ into (3.4) from the right.

${ }^{9}$ For the case where the fermionic oscillators contain the operator $K$, the commutator $[\underbrace{\hat{y}_{(1} \ldots \hat{y}_{1}}_{h_{1}-1+m} \underbrace{\hat{y}_{2} \ldots \hat{y}_{2)}}_{h_{1}-1-m}, \underbrace{\hat{y}_{(1} \ldots \hat{y}_{1}}_{h_{2}-\frac{1}{2}+\rho} \underbrace{\hat{y}_{2} \ldots \hat{y}_{2)}}_{h_{2}-\frac{1}{2}-\rho} K] K$ is the same as the one in (3.5). Then by multiplying the operator $K$ into (3.5) from the right, we obtain the corresponding commutator. Similarly, when we have the operator $K$ in both bosonic and fermionic oscillators, the commutator $[\underbrace{\hat{y}_{(1 \ldots \hat{y}}}_{h_{1}-1+m} \underbrace{\hat{y}_{2} \ldots \hat{y}_{2)}}_{h_{1}-1-m} K, \underbrace{\hat{y}_{(1} \ldots \hat{y}_{1}}_{h_{2}-\frac{1}{2}+\rho} \underbrace{\hat{y}_{2} \ldots \hat{y}_{2}}_{h_{2}-\frac{1}{2}-\rho} K] K$ with additional operator $K$ is the same as the one in (3.6). Again, by multiplying the operator $K$ into (3.6) from the right, we obtain the final result. 
Again the spins of right hand side are given by $\left(h_{1}+h_{2}-2 r+\frac{3}{2}\right)$ and $\left(h_{1}+h_{2}-2 r+\frac{1}{2}\right)$ respectively.

- The case-three with two fermionic oscillators. We consider the last case. For the case where there is no operator $K$ in the oscillator, the first equation of appendix (J.1) of [11] can be used. We shift the dummy variable $h \rightarrow r-1$. Then we obtain the following anticommutator $^{10}$

$$
\begin{aligned}
& \{\underbrace{\hat{y}_{(1} \ldots \hat{y}_{1}}_{h_{1}-\frac{1}{2}+\rho} \underbrace{\left.\hat{y}_{2} \ldots \hat{y}_{2}\right)}_{h_{1}-\frac{1}{2}-\rho}, \underbrace{\hat{y}_{(1} \ldots \hat{y}_{1}}_{h_{2}-\frac{1}{2}+\omega} \underbrace{\hat{y}_{2} \ldots \hat{y}_{2}}_{h_{2}-\frac{1}{2}-\omega}\}= \\
& -\frac{1}{2} \sum_{r=1}^{\left[\frac{h_{1}+h_{2}+1}{2}\right]}(-1)^{r}[\left(o_{\mathrm{F}, 2(r-1)}^{h_{1}+\frac{1}{2}, h_{2}+\frac{1}{2}}(\rho, \omega)+o_{\mathrm{B}, 2(r-1)}^{h_{1}+\frac{1}{2}, h_{2}+\frac{1}{2}}(\rho, \omega)\right) \underbrace{\hat{y}_{(1} \ldots \ldots \ldots \hat{y}_{1}}_{h_{1}+h_{2}-2 r+1+\rho+\omega} \underbrace{\left.\hat{y}_{2} \ldots \ldots \ldots \hat{y}_{2}\right)}_{h_{1}+h_{2}-2 r+1-\rho-\omega} \\
& -\left(o_{\mathrm{F}, 2(r-1)}^{h_{1}+\frac{1}{2}, h_{2}+\frac{1}{2}}(\rho, \omega)-o_{\mathrm{B}, 2(r-1)}^{h_{1}+\frac{1}{2}, h_{2}+\frac{1}{2}}(\rho, \omega)\right) \underbrace{\hat{y}_{(1} \ldots \ldots \ldots \hat{y}_{1}}_{h_{1}+h_{2}-2 r+1+\rho+\omega} \underbrace{\left.\hat{y}_{2} \ldots \ldots \ldots \hat{y}_{2}\right)}_{h_{1}+h_{2}-2 r+1-\rho-\omega} K] .
\end{aligned}
$$

The spin of the right hand side is given by $\left(h_{1}+h_{2}-2 r+2\right)$. The structure constants are given by appendix A.

Let us consider the next case where the operator $K$ appears in one of the oscillators. It turns out that

$$
\begin{aligned}
& \{\underbrace{\hat{y}_{(1} \ldots \hat{y}_{1}}_{h_{1}-\frac{1}{2}+\rho} \underbrace{\left.\hat{y}_{2} \ldots \hat{y}_{2}\right)}_{h_{1}-\frac{1}{2}-\rho}, \underbrace{\hat{y}_{(1} \ldots \hat{y}_{1}}_{h_{2}-\frac{1}{2}+\omega} \underbrace{\left.\hat{y}_{2} \ldots \hat{y}_{2}\right)}_{h_{2}-\frac{1}{2}-\omega} K\}= \\
& -\frac{\mathrm{i}}{2} \sum_{r=1}^{\left[\frac{h_{1}+h_{2}}{2}\right]}(-1)^{r}[\left(o_{\mathrm{F}, 2 r-1}^{h_{1}+\frac{1}{2}, h_{2}+\frac{1}{2}}(\rho, \omega)+o_{\mathrm{B}, 2 r-1}^{h_{1}+\frac{1}{2}, h_{2}+\frac{1}{2}}(\rho, \omega)\right) \underbrace{\hat{y}_{(1} \ldots \ldots \ldots \hat{y}_{1}}_{h_{1}+h_{2}-2 r+\rho+\omega} \underbrace{\left.\hat{y}_{2} \ldots \ldots \ldots \hat{y}_{2}\right)}_{h_{1}+h_{2}-2 r-\rho-\omega} \\
& -\left(o_{\mathrm{F}, 2 r-1}^{h_{1}+\frac{1}{2}, h_{2}+\frac{1}{2}}(\rho, \omega)-o_{\mathrm{B}, 2 r-1}^{h_{1}+\frac{1}{2}, h_{2}+\frac{1}{2}}(\rho, \omega)\right) \underbrace{\hat{y}_{(1} \ldots \ldots \ldots \hat{y}_{1}}_{h_{1}+h_{2}-2 r+\rho+\omega} \underbrace{\left.\hat{y}_{2} \ldots \ldots \ldots \hat{y}_{2} \ldots\right]}_{h_{1}+h_{2}-2 r-\rho-\omega} K,
\end{aligned}
$$

where the first equation of appendix (J.1) of [11] is used and dummy variable $h$ is replaced by $(r-1)$.

Therefore, there are nontrivial (anti)commutators (3.4), (3.5), (3.6), (3.7), and (3.8). See also appendices A and B where the structure constants are written in terms of generalized hypergeometric function. As an intermediate step for the $\mathcal{N}=4$ higher spin algebra $s h s_{2}[\mu]$, we should compute these for nonzero $\mu$.

\footnotetext{
${ }^{10}$ We can check that the anticommutator, $-\{\underbrace{\hat{y}_{(1 \ldots} \ldots \hat{y}_{1}}_{h_{1}-\frac{1}{2}+\rho} \underbrace{\left.\hat{y}_{2} \ldots \hat{y}_{2}\right)}_{h_{1}-\frac{1}{2}-\rho} K, \underbrace{\hat{y}_{(1} \ldots \hat{y}_{1}}_{h_{2}-\frac{1}{2}+\omega} \underbrace{\hat{y}_{2} \ldots \hat{y}_{2)}}_{h_{2}-\frac{1}{2}-\omega} K\}$, is the same
} as the one in (3.7) by similar analysis. 


\subsection{The (anti)commutators between the oscillators with nonzero $\mu$}

The result of [11] implies that although the structure constants contain linear $\mu$ factor, they will vanish under the wedge condition. See also appendices $(G .3)$ or $(G .4)$ of [11]. We realize that the field contents for the vanishing $\mu$ are the same as the ones for nonvanishing $\mu$. This implies that the mode dependent parts of any (anti)commutators remain the same. ${ }^{11}$ This reflects that in the OPE language of the $\mathcal{W}_{\infty}^{\mathcal{N}}=4[\mu]$ algebra, the field contents in the right hand side of OPE for the vanishing $\mu$ can be used for nonzero $\mu$. By shifting $h \rightarrow r-2$, we introduce the mode dependent function [28]

$$
\begin{aligned}
N_{r}^{h_{1}, h_{2}}(m, n) \equiv & \sum_{k=0}^{r-1}(-1)^{k}\left(\begin{array}{c}
r-1 \\
k
\end{array}\right) \\
& \times\left[h_{1}-1+m\right]_{r-1-k}\left[h_{1}-1-m\right]_{k}\left[h_{2}-1+n\right]_{k}\left[h_{2}-1-n\right]_{r-1-k} .
\end{aligned}
$$

The falling Pochhammer symbol $[a]_{n} \equiv a(a-1) \cdots(a-n+1)$ is used in (3.9).

- The case-one with two bosonic oscillators. After we take the same mode dependent part as the one (coming from $q_{F}$ or $q_{B}$ ) for vanishing $\mu$ case in (3.5), we introduce two $\mu$ dependent parts, $a_{2 r}^{h_{1}, h_{2}}(\nu)$ and $b_{2 r}^{h_{1}, h_{2}}(\nu)$. Note that there is a relation in (2.4). Once we have determined these two structure constants, then we are done. We can start with ${ }^{12}$

$$
\begin{aligned}
& {[\underbrace{\hat{y}_{(1} \ldots \hat{y}_{1}}_{h_{1}-1+m} \underbrace{\hat{y}_{2} \ldots \hat{y}_{2)}}_{h_{1}-1-m}, \underbrace{\hat{y}_{(1} \ldots \hat{y}_{1}}_{h_{2}-1+n} \underbrace{\left.\hat{y}_{2} \ldots \hat{y}_{2}\right)}_{h_{2}-1-n}]=} \\
& -\mathrm{i} \sum_{r=1}^{\left.\frac{h_{1}+h_{2}-1}{2}\right]}(-1)^{r} N_{2 r}^{h_{1} h_{2}(m, n)} \frac{1}{(2 r-1) !}[a_{2 r}^{h_{1}, h_{2}}(\nu) \underbrace{\hat{y}_{(1} \ldots \ldots \ldots \hat{y}_{1}}_{h_{1}+h_{2}-1-2 r+m+n} \underbrace{\left.\hat{y}_{2} \ldots \ldots \ldots \ldots \hat{y}_{2}\right)}_{h_{1}+h_{2}-1-2 r-m-n} \\
& -b_{2 r}^{h_{1}, h_{2}}(\nu) \underbrace{\hat{y}_{(1} \ldots \ldots \ldots . \hat{y}_{1}}_{h_{1}+h_{2}-1-2 r+m+n} \underbrace{\left.\hat{y}_{2} \ldots \ldots \ldots \ldots \hat{y}_{2}\right)}_{h_{1}+h_{2}-1-2 r-m-n} K] .
\end{aligned}
$$

We can compute the above commutators starting with $\left(h_{1}, h_{2}\right)=(2,2)$ with appropriate dummy variable $r$. One kind of the structure constants leads to the following $\mu$ (or $\nu$ ) dependent polynomials

$$
\begin{aligned}
& a_{2}^{2,2}=2, \quad a_{2}^{2,3}=2, \quad a_{2}^{2,4}=2, \quad a_{2}^{2,5}=2, \quad a_{2}^{2,6}=2, \quad a_{2}^{2,7}=2, \\
& a_{2}^{2,8}=2, \quad a_{2}^{2,9}=2, \quad a_{2}^{3,2}=2, \quad a_{2}^{3,3}=2, \quad a_{4}^{3,3}=-\frac{2}{15}\left(-15+\nu^{2}\right), \\
& a_{2}^{3,4}=2, \quad a_{4}^{3,4}=-\frac{2}{35}\left(-35+\nu^{2}\right), \quad a_{2}^{3,5}=2, \quad a_{4}^{3,5}=-\frac{2}{63}\left(-63+\nu^{2}\right), \\
& a_{2}^{3,6}=2, \quad a_{4}^{3,6}=-\frac{2}{99}\left(-99+\nu^{2}\right), \quad a_{2}^{3,7}=2, \quad a_{4}^{3,7}=-\frac{2}{143}\left(-143+\nu^{2}\right), \\
& a_{2}^{3,8}=2, \quad a_{4}^{3,8}=-\frac{2}{195}\left(-195+\nu^{2}\right), \quad a_{2}^{4,2}=2, \quad a_{2}^{4,3}=2,
\end{aligned}
$$

\footnotetext{
${ }^{11}$ We have checked that this validity is true up to total spin $11\left(h_{1}+h_{2} \leq 11\right)$. See also appendix $C$.

${ }^{12}$ We take the maximum value of $r$ as an infinity which should appear in the upper bound of the dummy variable $r$ of the summation over $r$ in this section and section 4 . For fixed $h_{1}, h_{2}$, most of the values for the $r$ which are greater than the sum of $\left(h_{1}+h_{2}\right)$ vanishes.
} 


$$
\begin{aligned}
& a_{4}^{4,3}=-\frac{2}{35}\left(-35+\nu^{2}\right), \quad a_{2}^{4,4}=2, \quad a_{4}^{4,4}=-\frac{2}{75}\left(-75+\nu^{2}\right), \\
& a_{6}^{4,4}=\frac{2}{525}(-5+\nu)(5+\nu)\left(-21+\nu^{2}\right), \quad a_{2}^{4,5}=2, \quad a_{4}^{4,5}=-\frac{2}{385}\left(-385+3 \nu^{2}\right), \\
& a_{6}^{4,5}=\frac{2}{2205}(-7+\nu)(7+\nu)\left(-45+\nu^{2}\right), \quad a_{2}^{4,6}=2, \quad a_{4}^{4,6}=-\frac{2}{195}\left(-195+\nu^{2}\right), \\
& a_{6}^{4,6}=\frac{2}{6237}(-9+\nu)(9+\nu)\left(-77+\nu^{2}\right), \quad a_{2}^{4,7}=2, \quad a_{4}^{4,7}=-\frac{2}{275}\left(-275+\nu^{2}\right), \\
& a_{6}^{4,7}=\frac{2}{14157}(-11+\nu)(11+\nu)\left(-117+\nu^{2}\right), \quad a_{2}^{5,2}=2, \quad a_{2}^{5,3}=2, \\
& a_{4}^{5,3}=-\frac{2}{63}\left(-63+\nu^{2}\right), \quad a_{2}^{5,4}=2, \quad a_{4}^{5,4}=-\frac{2}{385}\left(-385+3 \nu^{2}\right), \\
& a_{6}^{5,4}=\frac{2}{2205}(-7+\nu)(7+\nu)\left(-45+\nu^{2}\right), \quad a_{2}^{5,5}=2, a_{4}^{5,5}=-\frac{2}{637}\left(-637+3 \nu^{2}\right), \\
& a_{6}^{5,5}=\frac{2}{24255}\left(24255-562 \nu^{2}+3 \nu^{4}\right), \\
& a_{8}^{5,5}=-\frac{2}{33075}(-7+\nu)(-5+\nu)(5+\nu)(7+\nu)\left(-27+\nu^{2}\right), \quad a_{2}^{5,6}=2, \\
& a_{4}^{5,6}=-\frac{2}{315}\left(-315+\nu^{2}\right), \quad a_{6}^{5,6}=\frac{2}{63063}\left(63063-922 \nu^{2}+3 \nu^{4}\right), \\
& a_{8}^{5,6}=\frac{2}{218295}(-9+\nu)(-7+\nu)(7+\nu)(9+\nu)\left(-55+\nu^{2}\right), \quad a_{2}^{6,2}=2, a_{2}^{6,3}=2, \\
& a_{4}^{6,3}=-\frac{2}{99}\left(-99+\nu^{2}\right), \quad a_{2}^{6,4}=2, \quad a_{4}^{6,4}=-\frac{2}{195}\left(-195+\nu^{2}\right), \\
& a_{6}^{6,4}=\frac{2}{6237}(-9+\nu)(9+\nu)\left(-77+\nu^{2}\right), \quad a_{2}^{6,5}=2, \quad a_{4}^{6,5}=-\frac{2}{315}\left(-315+\nu^{2}\right), \\
& a_{6}^{6,5}=\frac{2}{63063}\left(63063-922 \nu^{2}+3 \nu^{4}\right) \text {, } \\
& a_{8}^{6,5}=-\frac{2}{218295}(-9+\nu)(-7+\nu)(7+\nu)(9+\nu)\left(-55+\nu^{2}\right), \quad a_{2}^{7,2}=2, a_{2}^{7,3}=2, \\
& a_{4}^{7,3}=-\frac{2}{143}\left(-143+\nu^{2}\right), \quad a_{2}^{7,4}=2, \quad a_{4}^{7,4}=-\frac{2}{275}\left(-275+\nu^{2}\right), \\
& a_{6}^{7,4}=\frac{2}{14157}(-11+\nu)(11+\nu)\left(-117+\nu^{2}\right), \quad a_{2}^{8,2}=2, \quad a_{2}^{8,3}=2, \\
& a_{4}^{8,3}=-\frac{2}{195}\left(-195+\nu^{2}\right), \quad a_{2}^{9,2}=2 .
\end{aligned}
$$

First of all, the $\nu$ independent constant coefficients appear as 2. Let us keep track of the $\nu$ dependence. The $\nu^{2}$ dependence appears in the coefficient $a_{4}^{3,3}$. This behavior goes to $a_{4}^{3,8}$ by varying $h_{2}$ for fixed $h_{1}$ and $2 r$. The $\nu^{4}$ dependence appears in the coefficient $a_{6}^{4,4}$. This behavior appears until the coefficient $a_{6}^{4,7}$ for fixed $h_{1}$ and $2 r$. Moreover, $\nu^{6}$ dependence appears in the coefficient $a_{8}^{5,5}$. This behavior appears until the coefficient $a_{8}^{5,6}$ for fixed $h_{1}$ and $2 r$.

By considering the constant piece of the coefficient $a_{4}^{3,3}$ further, then $\left(\nu^{2}-15\right)$ can be written in terms of $(\nu-3)(\nu+5)$ plus other term. By changing as $\nu \rightarrow-\nu$, then we have $(\nu-5)(\nu+3)$ which is equivalent to change the signs in the constant terms in the original expression. That is -3 goes to +3 and +5 goes to -5 . By adding these two, we obtain $2\left(\nu^{2}-15\right)$ which is proportional to the previous expression. We will observe that the factor $(\nu-3)(\nu+5)$ can be written in terms of the linear combination of two generalized 
hypergeometric functions at the specific upper and lower arguments. We can analyze each coefficient $a_{4}^{3, h_{2}}$ term where $h_{2}=4,5,6,7,8$ by adding the number 2 in the constant terms at each step. Then for the coefficient $a_{4}^{3,8}$, we can consider the two factors, $(\nu-13)(\nu+15)$ which can be seen from the above $(\nu-3)(\nu+5)$ after five steps and $(\nu-15)(\nu+13)$ which can be obtained by sign changes. By adding these two factors, we obtain the coefficient $a_{4}^{3,8}$ up to an overall scale. ${ }^{13}$

We can consider the numerical value 21 as the product of odd numbers 3 and 7 in the coefficient $a_{6}^{4,4}$. This implies that we consider the factor $(\nu-3)(\nu+5)(\nu-5)(\nu+7)$ which is the multiplication between the previous factor $(\nu-3)(\nu+5)$ appeared in previous paragraph and $(\nu-5)(\nu+7)$ which is obtained by adding the number 2 to each constant term in the first factor (ignore the signs). Furthermore, we consider the next quartic term $(\nu+3)(\nu-5)(\nu+5)(\nu-7)$ where the first two factors can be seen from the previous paragraph and the next two factors are obtained by adding the number 2 to the constant terms in the first two factors. Then we realize that by adding these two quartic terms we have the coefficient $a_{6}^{4,4}$ up to an overall scale. The quartic behavior comes from the fact that the spins $h_{1}, h_{2}$ and $r$ are increased. It is easy to observe that the relevant quartic terms for the coefficient $a_{6}^{4,7}$ are given by the factor $(\nu-9)(\nu+11)(\nu-11)(\nu+13)$ where $117=9 \times 13$ and the factor $(\nu+9)(\nu-11)(\nu+11)(\nu-13) .{ }^{14}$

Let us analyze the sextic term in the coefficient $a_{8}^{5,5}$ where the spins $h_{1}, h_{2}$ and $r$ are further increased. In this case, we reconsider 27 as the multiple of 3 and 9 . Then we have $(\nu-3)(\nu+5)(\nu-5)(\nu+7)(\nu-7)(\nu+9)$ where the first and last factors come from $\left(\nu^{2}-27\right)$. We understand that the above sextic term can be written in terms of the first quartic term which appears in previous paragraph and the factor $(\nu-7)(\nu+9)$ which is obtained by adding the number 2 from the factor $(\nu-5)(\nu+7)$. The other sextic term can be seen from the above by changing $\nu \rightarrow-\nu$ and is $(\nu+3)(\nu-5)(\nu+5)(\nu-7)(\nu+7)(\nu-9)$ (or simply by changing the signs in the constant terms). Therefore, by adding these two sextic terms we obtain the coefficient $a_{8}^{5,5}$ up an overall scale. For the coefficient $a_{8}^{5,6}$, by realizing that 55 is the product of 5 and 11 , the relevant sextic terms are $(\nu \pm 5)(\nu \mp 7)(\nu \pm 7)(\nu \mp$ $9)(\nu \pm 9)(\nu \mp 11) \cdot{ }^{15}$

Although it is not obvious, at the moment, how we can rearrange for the coefficients $a_{4}^{4,4}$ and $a_{4}^{4,5}$ because the constant terms cannot be written as the product of two neighboring odd numbers, once we determine the $\mu$ dependence for the above descriptions where we have nice factorized forms, then these exotic coefficients also behave nicely.

The other kind of structure constants gives

$$
\begin{aligned}
& b_{4}^{3,3}=-\frac{4 \nu}{15}, \quad b_{4}^{3,4}=-\frac{4 \nu}{35}, \quad b_{4}^{3,5}=-\frac{4 \nu}{63}, \quad b_{4}^{3,6}=-\frac{4 \nu}{99}, b_{4}^{3,7}=-\frac{4 \nu}{143}, \\
& b_{4}^{3,8}=-\frac{4 \nu}{195}, \quad b_{4}^{4,3}=-\frac{4 \nu}{35}, \quad b_{4}^{4,4}=-\frac{4 \nu}{75}, \quad b_{6}^{4,4}=\frac{8}{525}(-5+\nu) \nu(5+\nu),
\end{aligned}
$$

\footnotetext{
${ }^{13}$ Explicitly we have $\phi_{4}^{3,3}(\mu, 1)=-\frac{1}{15}(-3+\nu)(5+\nu)$ by using the definition of (4.1) with (2.4) and $\phi_{4}^{3,3}(1-\mu, 1)=\left.\phi_{4}^{3,3}(\mu, 1)\right|_{\nu, 4}$

${ }^{14}$ In this case we have $\phi_{6}^{4,4}(\mu, 1)=\frac{1}{525}(-5+\nu)(-3+\nu)(5+\nu)(7+\nu)$ with $(4.1)$ and $(2.4)$ and also we have $\phi_{6}^{4,4}(1-\mu, 1)=\left.\phi_{6}^{4,4}(\mu, 1)\right|_{\nu}$

${ }^{15}$ Similarly, we have $\phi_{8}^{5,5}(\mu, 1)=\frac{1}{33075}(-7+\nu)(-5+\nu)(-3+\nu)(5+\nu)(7+\nu)(9+\nu)$ together with (4.1) and we have $\phi_{8}^{5,5}(1-\mu, 1)=\left.\phi_{8}^{5,5}(\mu, 1)\right|_{\nu \rightarrow-\nu}$.
} 


$$
\begin{aligned}
& b_{4}^{4,5}=-\frac{12 \nu}{385}, \quad b_{6}^{4,5}=\frac{8}{2205}(-7+\nu) \nu(7+\nu), \quad b_{4}^{4,6}=-\frac{4 \nu}{195}, \\
& b_{6}^{4,6}=\frac{8}{6237}(-9+\nu) \nu(9+\nu), \quad b_{4}^{4,7}=-\frac{4 \nu}{275}, b_{6}^{4,7}=\frac{8}{14157}(-11+\nu) \nu(11+\nu), \\
& b_{4}^{5,3}=-\frac{4 \nu}{63}, \quad b_{4}^{5,4}=-\frac{12 \nu}{385}, \quad b_{6}^{5,4}=\frac{8}{2205}(-7+\nu) \nu(7+\nu), \quad b_{4}^{5,5}=-\frac{12 \nu}{637}, \\
& b_{6}^{5,5}=\frac{8}{24255} \nu\left(-287+3 \nu^{2}\right), \quad b_{8}^{5,5}=-\frac{4}{11025}(-7+\nu)(-5+\nu) \nu(5+\nu)(7+\nu), \\
& b_{4}^{5,6}=-\frac{4 \nu}{315}, \quad b_{6}^{5,6}=\frac{8}{63063} \nu\left(-467+3 \nu^{2}\right), \\
& b_{8}^{5,6}=-\frac{4}{72765}(-9+\nu)(-7+\nu) \nu(7+\nu)(9+\nu), \quad b_{4}^{6,3}=-\frac{4 \nu}{99}, \quad b_{4}^{6,4}=-\frac{4 \nu}{195}, \\
& b_{6}^{6,4}=\frac{8}{6237}(-9+\nu) \nu(9+\nu), \quad b_{4}^{6,5}=-\frac{4 \nu}{315}, \quad b_{6}^{6,5}=\frac{8}{63063} \nu\left(-467+3 \nu^{2}\right), \\
& b_{8}^{6,5}=-\frac{4}{72765}(-9+\nu)(-7+\nu) \nu(7+\nu)(9+\nu), \quad b_{4}^{7,3}=-\frac{4 \nu}{143}, b_{4}^{7,4}=-\frac{4 \nu}{275}, \\
& b_{6}^{7,4}=\frac{8}{14157}(-11+\nu) \nu(11+\nu), \quad b_{4}^{8,3}=-\frac{4 \nu}{195} .
\end{aligned}
$$

There are no numerical constants. All the coefficients depend on $\nu$ (At $\nu=0$, these are vanishing). The $\nu$ dependence appears in the coefficient $b_{4}^{3,3}$. This behavior goes to $b_{4}^{3,8}$ by varying the $h_{2}$ for fixed $h_{1}$ and $2 r$. It is not straightforward to analyze these cases because they do depend on the linear $\nu$ with different numerical coefficients.

The $\nu^{3}$ dependence appears in the coefficient $b_{6}^{4,4}$. This behavior appears until the coefficient $b_{6}^{4,7}$ for fixed $h_{1}$ and $2 r$. Let us look at the coefficient $b_{6}^{4,4}$ which has $(-5+$ $\nu) \nu(5+\nu)$. We saw the quartic terms are given by $(\nu \mp 3)(\nu \pm 5)(\nu \mp 5)(\nu \pm 7)$ in the coefficient of $a_{6}^{4,4}$. Note that the middle two factors are the same for each quartic term. Then by subtracting these two, we obtain $8 \nu(\nu-5)(\nu+5)$ which is related to the above coefficient. Similarly, from the quartic terms $(\nu \mp 9)(\nu \pm 11)(\nu \mp 11)(\nu \pm 13)$ in the coefficient of $a_{6}^{4,7}$, by subtracting them, we arrive at $8 \nu(\nu-11)(\nu+11)$ which is related to the coefficient $b_{6}^{4,7}$. This implies that the second kind of structure constants can be obtained by taking the subtraction between two generalized hypergeometric functions.

Moreover, $\nu^{5}$ dependence appears in the coefficient $b_{8}^{5,5}$. This behavior appears until the coefficient $b_{8}^{5,6}$ for fixed $h_{1}$ and $2 r$. We can analyze similarly. From the sextic terms $(\nu \pm 3)(\nu \mp 5)(\nu \pm 5)(\nu \mp 7)(\nu \pm 7)(\nu \mp 9)$ in the coefficient of $a_{8}^{5,5}$, we can take the difference between them and obtain $12 \nu(\nu-5)(\nu+5)(\nu-7)(\nu+7)$ which is proportional to the coefficient $b_{8}^{5,5}$. From the sextic terms $(\nu \pm 5)(\nu \mp 7)(\nu \pm 7)(\nu \mp 9)(\nu \pm 9)(\nu \mp 11)$ in the coefficient of $a_{8}^{5,6}$, we can take the difference between them and obtain $12 \nu(\nu-7)(\nu+7)(\nu-$ $9)(\nu+9)$ which is related to the coefficient $b_{8}^{5,6}$.

Then the question is how we generalize the above structure constants for generic $h_{1}, h_{2}$ and $r$. Recall that the form of $\mu$ dependence of (3.4), (3.5), (3.6), (3.7), and (3.8) is the combination of two generalized hypergeometric functions. The question is how the $\mu$ or $\nu$ dependence appears in the structure constant. Remember that the upper four elements of generalized hypergeometric function appear in the numerator of the fractional coefficient while the lower three elements appear in the denominator of the fractional coefficient. The behaviors of (3.11) and (3.12) imply that the deformation parameter $\nu$ (or $\mu$ ) can appear 
in the upper four elements of generalized hypergeometric function. The simplest case we can consider is to put the deformation parameter into the first two elements respectively while we do not touch the third and fourth elements which are associated with the dummy variable $r$ (Note that we can choose other two elements rather than first two because from the definition of generalized hypergeometric function eq. (A.3) the assignments of four upper elements are arbitrary.).

It turns out that the $\mu$ dependent structure constants can be written in terms of generalized hypergeometric functions as follows:

$$
\begin{aligned}
a_{2 r}^{h_{1}, h_{2}}(\mu)= & { }_{4} F_{3}\left[\begin{array}{c}
\frac{1}{2}+\mu, \frac{1}{2}-\mu, \frac{2-2 r}{2}, \frac{1-2 r}{2} \\
\frac{3}{2}-h_{1}, \frac{3}{2}-h_{2}, \frac{1}{2}+h_{1}+h_{2}-2 r
\end{array} \mid 1\right] \\
& +{ }_{4} F_{3}\left[\begin{array}{c}
\frac{3}{2}-\mu,-\frac{1}{2}+\mu, \frac{2-2 r}{2}, \frac{1-2 r}{2} \\
\frac{3}{2}-h_{1}, \frac{3}{2}-h_{2}, \frac{1}{2}+h_{1}+h_{2}-2 r \mid 1
\end{array} \mid,\right. \\
b_{2 r}^{h_{1}, h_{2}}(\mu)= & { }_{4} F_{3}\left[\begin{array}{c}
\frac{1}{2}+\mu, \frac{1}{2}-\mu, \frac{2-2 r}{2}, \frac{1-2 r}{2} \\
\frac{3}{2}-h_{1}, \frac{3}{2}-h_{2}, \frac{1}{2}+h_{1}+h_{2}-2 r
\end{array} \mid 1\right] \\
& -{ }_{4} F_{3}\left[\begin{array}{c}
\frac{3}{2}-\mu,-\frac{1}{2}+\mu, \frac{2-2 r}{2}, \frac{1-2 r}{2} \\
\frac{3}{2}-h_{1}, \frac{3}{2}-h_{2}, \frac{1}{2}+h_{1}+h_{2}-2 r \mid
\end{array}\right] .
\end{aligned}
$$

Note that by taking $\mu \rightarrow(1-\mu$ ) (or $\nu \rightarrow-\nu$ ) in the second generalized hypergeometric function in each case, we obtain the first one. They have common elements, the last two in the upper and the three in the lower. We will see that the above two structure constants are denoted by the first one in (4.2). Of course, the previous explicit results in (3.11) and (3.12) satisfy the relation (3.13). Note that the infinite series of generalized hypergeometric function terminates if any of upper elements is negative integer or zero. It is obvious that the common upper third element of generalized hypergeometric functions in $(3.13), \frac{(2-2 r)}{2}$, becomes negative integer or zero for (3.11) and (3.12). Furthermore, the sum of upper elements plus 1 is equal to the sum of lower elements of generalized hypergeometric functions which is called "saalschutzian" [28]. In this case, there exists some relation which connects two different generalized hypergeometric functions. We can analyze the other two cases which will be described in appendix C.

\section{The $\mathcal{N}=4$ higher spin algebra $\operatorname{shs}_{2}[\mu]$}

\subsection{Mode dependent structure constants}

From the results of previous section, we realize that the third and fourth upper elements of generalized hypergeometric function for the case-two and case-three differ from the one for the case-one. See also appendix C. By introducing the notation for the generalized hypergeometric function ${ }^{16}$

$$
\phi_{r}^{h_{1}, h_{2}}(\Lambda, a) \equiv_{4} F_{3}\left[\begin{array}{c}
\frac{1}{2}+\Lambda, \frac{1}{2}-\Lambda, \frac{1+a-r}{2}, \frac{a-r}{2} \\
\frac{3}{2}-h_{1}, \frac{3}{2}-h_{2}, \frac{1}{2}+h_{1}+h_{2}-r
\end{array} \mid 1\right],
$$

\footnotetext{
${ }^{16}$ Note that there are relations in (2.4). We can present the structure constants by using $\mu$ or $\nu$. For the $\nu$, we replace $\mu$ by $\frac{(\nu+1)}{2}$. This leads to the changing $\nu \leftrightarrow-\nu$ for the shift $\mu \leftrightarrow 1-\mu$.
} 
where the sum of upper four elements plus 1 is not equal to the sum of lower three elements unless $a=1$ and mode dependent function in (3.9), we can write down the mode dependent structure constants which are polynomials of $\mu$ as follows:

$$
\begin{aligned}
\mathrm{BB}_{r, \pm}^{h_{1}, h_{2}}(m, n ; \mu) \equiv-\frac{1}{(r-1) !} N_{r}^{h_{1}, h_{2}}(m, n)\left[\phi_{r}^{h_{1}, h_{2}}(\mu, 1) \pm \phi_{r}^{h_{1}, h_{2}}(1-\mu, 1)\right] \\
\mathrm{BF}_{r, \pm}^{h_{1}, h_{2}+\frac{1}{2}}(m, \rho ; \mu) \equiv-\frac{1}{(r-1) !} N_{r}^{h_{1}, h_{2}+\frac{1}{2}}(m, \rho)\left[\phi_{r+1}^{h_{1}, h_{2}+1}\left(\mu, \frac{3 \pm 1}{2}\right)\right. \\
\left. \pm \phi_{r+1}^{h_{1}, h_{2}+1}\left(1-\mu, \frac{3 \pm 1}{2}\right)\right] \\
\mathrm{FF}_{r, \pm}^{h_{1}+\frac{1}{2}, h_{2}+\frac{1}{2}}(\rho, \omega ; \mu) \equiv-\frac{1}{(r-1) !} N_{r}^{h_{1}+\frac{1}{2}, h_{2}+\frac{1}{2}}(\rho, \omega)\left[\phi_{r+1}^{h_{1}+1, h_{2}+1}\left(\mu, \frac{3 \pm 1}{2}\right)\right. \\
\left. \pm \phi_{r+1}^{h_{1}+1, h_{2}+1}\left(1-\mu, \frac{3 \pm 1}{2}\right)\right] .
\end{aligned}
$$

From the expression of (4.1), although by increasing the index $r$ as $r+1$ for fixed $h_{1}$ and $h_{2}$, the third lower element is changed, we can make the last two upper elements remain the same by further change of $a$ as $a+1$. In this case, the number of terms in the generalized hypergeometric functions in eq. (A.3) are the same while its coefficients are different (from the different lower elements) and the highest power of $\mu$ is the same.

Moreover, by changing $a$ as $a+1$ for fixed $h_{1}, h_{2}$ and $r$, we observe that the third upper element in the former is the same as the fourth upper element in the latter because the third element is given by the four element plus $\frac{1}{2}$. For example, when $a=1$ with even $r$, we have negative integer in the third upper element and this will terminate the generalized hypergeometric function in eq. (A.3). Therefore, the two cases, $a=1$ and $a=2$ for the same $h_{1}, h_{2}, r$ values, will have the same number of terms (the higher power of $\mu$ is the same) with its different coefficients.

Let us emphasize that these structure constants appear in the $\mathcal{N}=4$ higher spin generators of spins (equal to the sum of upper indices minus lower index) in the right hand side of (anti)commutators. Note that in the second and third structure constants of (4.2), the three indices (upper two and lower one) in the generalized hypergeometric functions appear differently: $h_{2}$ by $h_{2}+\frac{1}{2}$ and $r$ by $r+1$ for the former (and $h_{i}$ by $h_{i}+\frac{1}{2}$ and $r$ by $r+1$ for the latter). For the lower signs of these structure constants, the second argument of (4.1) which is equal to 1 is the same as the one of first structure constant of (4.2) although the upper and lower indices are different from each other.

We have the following symmetry under the transformation $\mu \leftrightarrow 1-\mu$ (corresponding to $N \leftrightarrow k$ symmetry in the $\mathcal{N}=4$ coset model $[1,59])$

$$
\begin{aligned}
\mathrm{BB}_{r, \pm}^{h_{1}, h_{2}}(m, n ; \mu) & = \pm \mathrm{BB}_{r, \pm}^{h_{1}, h_{2}}(m, n ; 1-\mu), \\
\mathrm{BF}_{r, \pm}^{h_{1}, h_{2}+\frac{1}{2}}(m, \rho ; \mu) & = \pm \mathrm{BF}_{r, \pm}^{h_{1}, h_{2}+\frac{1}{2}}(m, \rho ; 1-\mu), \\
\mathrm{FF}_{r, \pm}^{h_{1}+\frac{1}{2}, h_{2}+\frac{1}{2}}(\rho, \omega ; \mu) & = \pm \mathrm{FF}_{r, \pm}^{h_{1}+\frac{1}{2}, h_{2}+\frac{1}{2}}(\rho, \omega ; 1-\mu) .
\end{aligned}
$$


We observe that the half of the structure constants from (4.3) vanish at $\mu=\frac{1}{2}$ (or $\nu=0$ which is the undeformed case). We can write down the mode dependent structure constants for vanishing $\mu$ and they can be related to the ones in Odake basis [25] as follows:

$$
\begin{aligned}
\mathrm{BB}_{h+2, \pm}^{h_{1}, h_{2}}(m, n ; 0) & =-2\left(p_{\mathrm{F}, h}^{h_{1}, h_{2}}(m, n) \pm p_{\mathrm{B}, h}^{h_{1}, h_{2}}(m, n)\right) \\
\mathrm{BF}_{2(h+1),+}^{h_{1}, h_{2}+\frac{1}{2}}(m, \rho ; 0) & =-4\left(q_{\mathrm{F}, 2 h}^{h_{1}, h_{2}+\frac{1}{2}}(m, \rho)+q_{\mathrm{B}, 2 h}^{h_{1}, h_{2}+\frac{1}{2}}(m, \rho)\right) \\
\mathrm{BF}_{2(h+1),-}^{h_{1}, h_{2}+\frac{1}{2}}(m, \rho ; 0) & =\frac{8(h+1)}{\left(2 h_{1}-2 h-3\right)}\left(q_{\mathrm{F}, 2 h}^{h_{1}, h_{2}+\frac{1}{2}}(m, \rho)-q_{\mathrm{B}, 2 h}^{h_{1}, h_{2}+\frac{1}{2}}(m, \rho)\right) \\
\mathrm{BF}_{2 h+3,+}^{h_{1}, h_{2}+\frac{1}{2}}(m, \rho ; 0) & =4\left(q_{\mathrm{F}, 2 h+1}^{h_{1}, h_{2}+\frac{1}{2}}(m, \rho)-q_{\mathrm{B}, 2 h+1}^{h_{1}, h_{2}+\frac{1}{2}}(m, \rho)\right) \\
\mathrm{BF}_{2 h+3,-}^{h_{1}, h_{2}+\frac{1}{2}}(m, \rho ; 0) & =-\frac{2(2 h+3)}{\left(h_{1}-h-2\right)}\left(q_{\mathrm{F}, 2 h+1}^{h_{1}, h_{2}+\frac{1}{2}}(m, \rho)+q_{\mathrm{B}, 2 h+1}^{h_{1}, h_{2}+\frac{1}{2}}(m, \rho)\right) \\
\mathrm{FF}_{2 h+1,+}^{h_{1}+\frac{1}{2}, h_{2}+\frac{1}{2}}(\rho, \omega ; 0) & =-\frac{1}{2}\left(o_{\mathrm{F}, 2 h}^{h_{1}+\frac{1}{2}, h_{2}+\frac{1}{2}}(\rho, \omega)+o_{\mathrm{B}, 2 h}^{h_{1}+\frac{1}{2}, h_{2}+\frac{1}{2}}(\rho, \omega)\right) \\
\mathrm{FF}_{2 h+1,-}^{h_{1}+\frac{1}{2}, h_{2}+\frac{1}{2}}(\rho, \omega ; 0) & =-\frac{(2 h+1)}{4\left(h_{1}+h_{2}-h\right)}\left(o_{\mathrm{F}, 2 h}^{h_{1}+\frac{1}{2}, h_{2}+\frac{1}{2}}(\rho, \omega)-o_{\mathrm{B}, 2 h}^{h_{1}+\frac{1}{2}, h_{2}+\frac{1}{2}}(\rho, \omega)\right) \\
\mathrm{FF}_{2(h+1),+}^{h_{1}+\frac{1}{2}, h_{2}+\frac{1}{2}}(\rho, \omega ; 0) & =\frac{1}{2}\left(o_{\mathrm{F}, 2 h+1}^{h_{1}+\frac{1}{2}, h_{2}+\frac{1}{2}}(\rho, \omega)-o_{\mathrm{B}, 2 h+1}^{h_{1}+\frac{1}{2}, h_{2}+\frac{1}{2}}(\rho, \omega)\right), \\
\mathrm{FF}_{2(h+1),-}^{h_{1}+\frac{1}{2}, h_{2}+\frac{1}{2}}(\rho, \omega ; 0) & =\frac{(h+1)}{2\left(h_{1}+h_{2}-h\right)-1}\left(o_{\mathrm{F}, 2 h+1}^{h_{1}+\frac{1}{2}, h_{2}+\frac{1}{2}}(\rho, \omega)+o_{\mathrm{B}, 2 h+1}^{h_{1}+\frac{1}{2}, h_{2}+\frac{1}{2}}(\rho, \omega)\right) .
\end{aligned}
$$

In other words, the six independent structure constants can be written in terms of those in (4.2). Note that there are shifts between the left hand side and the right hand side in the lower spin indices in (4.4).

- The case-one with two bosonic oscillators. We can rewrite (3.10) with the help of (3.13) (or from eq. (D.1)) as follows:

$$
\begin{aligned}
& {[\underbrace{\hat{y}_{(1} \ldots \hat{y}_{1}}_{h_{1}-1+m} \underbrace{\hat{y}_{2} \ldots \hat{y}_{2)}}_{h_{1}-1-m}, \underbrace{\hat{y}_{(1} \ldots \hat{y}_{1}}_{h_{2}-1+n} \underbrace{\left.\hat{y}_{2} \ldots \hat{y}_{2}\right)}_{h_{2}-1-n}]} \\
& =\mathrm{i} \sum_{r=1}^{\left[\frac{h_{1}+h_{2}-1}{2}\right]}(-1)^{r}[\mathrm{BB}_{2 r,+}^{h_{1}, h_{2}}(m, n ; \mu) \underbrace{\hat{y}_{(1} \ldots \ldots \ldots \ldots \hat{y}_{1}}_{h_{1}+h_{2}-2 r-1+m+n} \underbrace{\left.\hat{y}_{2} \ldots \ldots \ldots \ldots \hat{y}_{2}\right)}_{h_{1}+h_{2}-2 r-1-m-n} \\
& -\mathrm{BB}_{2 r,-}^{h_{1}, h_{2}}(m, n ; \mu) \underbrace{\hat{y}_{(1} \ldots \ldots \ldots \ldots \hat{y}_{1}}_{h_{1}+h_{2}-2 r-1+m+n} \underbrace{\hat{y}_{2} \ldots \ldots \ldots \ldots \hat{y}_{2}}_{h_{1}+h_{2}-2 r-1-m-n} K] \text {. }
\end{aligned}
$$

The only difference between the first structure constant and the second one is the sign in the generalized hypergeometric function containing the argument $(1-\mu)$ from (4.2). 
- The case-two with one bosonic and one fermionic oscillators. From eq. (D.2), we can reexpress this commutator as follows:

$$
\begin{aligned}
& {[\underbrace{\hat{y}_{(1} \ldots \hat{y}_{1}}_{h_{1}-1+m} \underbrace{\left.\hat{y}_{2} \ldots \hat{y}_{2}\right)}_{h_{1}-1-m}, \underbrace{\hat{y}_{(1} \ldots \hat{y}_{1}}_{h_{2}-\frac{1}{2}+\rho} \underbrace{\hat{y}_{2} \ldots \hat{y}_{2)}}_{h_{2}-\frac{1}{2}-\rho}]} \\
& =\sum_{r=1}^{\left[\frac{h_{1}+h_{2}-1}{2}\right]}[\mathrm{i}(-1)^{r} \mathrm{BF}_{2 r,+}^{h_{1}, h_{2}+\frac{1}{2}}(m, \rho ; \mu) \underbrace{\hat{y}_{(1} \ldots \ldots \ldots \ldots \hat{y}_{1}}_{h_{1}+h_{2}-2 r-\frac{1}{2}+m+\rho} \underbrace{\left.\hat{y}_{2} \ldots \ldots \ldots \ldots \hat{y}_{2}\right)}_{h_{1}+h_{2}-2 r-\frac{1}{2}-m-\rho}] \\
& \quad-\sum_{r=1}^{\left[\frac{h_{1}+h_{2}}{2}\right]}[(-1)^{r} \frac{2\left(h_{1}-r\right)}{2 r-1} \mathrm{BF}_{2 r-1,-}^{h_{1}, h_{2}+\frac{1}{2}}(m, \rho ; \mu) \underbrace{\hat{y}_{(1 \ldots \ldots . t} \ldots \ldots \ldots \hat{y}_{1}}_{h_{1}+h_{2}-2 r+\frac{1}{2}+m+\rho} \underbrace{\left.\hat{y}_{2} \ldots \ldots \ldots \ldots \hat{y}_{2}\right)}_{h_{1}+h_{2}-2 r+\frac{1}{2}-m-\rho} K] .
\end{aligned}
$$

From eq. (D.9), we can reexpress by using the above mode dependent structure constant

$$
\begin{aligned}
& {[\underbrace{\hat{y}_{(1} \ldots \hat{y}_{1}}_{h_{1}-1+m} \underbrace{\hat{y}_{2} \ldots \hat{y}_{2)}}_{h_{1}-1-m} K, \underbrace{\hat{y}_{\left(1 \ldots \hat{y}_{1}\right.}}_{h_{2}-\frac{1}{2}+\rho} \underbrace{\hat{y}_{2} \ldots \hat{y}_{2)}}_{h_{2}-\frac{1}{2}-\rho}]} \\
& =-\sum_{r=1}^{\left[\frac{h_{1}+h_{2}}{2}\right]}[(-1)^{r} \mathrm{BF}_{2 r-1,+}^{h_{1}, h_{2}+\frac{1}{2}}(m, \rho ; \mu) \underbrace{\hat{y}_{(1} \ldots \ldots \ldots \ldots . \hat{y}_{1}}_{h_{1}+h_{2}-2 r+\frac{1}{2}+m+\rho} \underbrace{\left.\hat{y}_{2} \ldots \ldots \ldots \ldots \hat{y}_{2}\right)}_{h_{1}+h_{2}-2 r+\frac{1}{2}-m-\rho} K] \\
& \quad+\sum_{r=1}^{\left[\frac{h_{1}+h_{2}-1}{2}\right]}[(-1)^{r} \frac{\hat{i}^{\left(h_{1}-r-\frac{1}{2}\right)}}{r} \mathrm{BF}_{2 r,-}^{h_{1}, h_{2}+\frac{1}{2}}(m, \rho ; \mu) \underbrace{\hat{y}_{(1} \ldots \ldots \ldots \ldots \hat{y}_{1}}_{h_{1}+h_{2}-2 r-\frac{1}{2}+m+\rho} \underbrace{\hat{y}_{2} \ldots \ldots \ldots \ldots \hat{y}_{2)}}_{h_{1}+h_{2}-2 r-\frac{1}{2}-m-\rho}] .
\end{aligned}
$$

- The case-three with two fermionic oscillators. The following expression can be obtained by considering eq. (D.10)

$$
\begin{aligned}
&\{\underbrace{\hat{y}_{(1} \ldots \hat{y}_{1}}_{h_{1}-\frac{1}{2}+\rho} \underbrace{\left.\hat{y}_{2} \ldots \hat{y}_{2}\right)}_{h_{1}-\frac{1}{2}-\rho}, \underbrace{\hat{y}_{(1} \ldots \hat{y}_{1}}_{h_{2}-\frac{1}{2}+\omega} \underbrace{\left.\hat{y}_{2} \ldots \hat{y}_{2}\right)}_{h_{2}-\frac{1}{2}-\omega}\} \\
&=\sum_{r=1}^{\left.\frac{h_{1}+h_{2}+1}{2}\right]}(-1)^{r}[\mathrm{FF}_{2 r-1,+}^{h_{1}+\frac{1}{2}, h_{2}+\frac{1}{2}}(\rho, \omega ; \mu) \underbrace{\hat{y}_{(1} \ldots \ldots \ldots \hat{y}_{1}}_{h_{1}+h_{2}-2 r+1+\rho+\omega} \underbrace{\left.\hat{y}_{2} \ldots \ldots \ldots \hat{y}_{2}\right)}_{h_{1}+h_{2}-2 r+1-\rho-\omega} \\
&-\frac{2\left(h_{1}+h_{2}-r+1\right)}{2 r-1} \mathrm{FF}_{2 r-1,-}^{h_{1}+\frac{1}{2}, h_{2}+\frac{1}{2}}(\rho, \omega ; \mu) \underbrace{\hat{y}_{(1} \ldots \ldots \ldots \hat{y}_{1}}_{h_{1}+h_{2}-2 r+1+\rho+\omega} \underbrace{\hat{y}_{2} \ldots \ldots \ldots \hat{y}_{2}}_{h_{1}+h_{2}-2 r+1-\rho-\omega} K] .
\end{aligned}
$$


We have the following expression by considering eq. (D.13)

$$
\begin{aligned}
& \{\underbrace{\hat{y}_{(1} \ldots \hat{y}_{1}}_{h_{1}-\frac{1}{2}+\rho} \underbrace{\left.\hat{y}_{2} \ldots \hat{y}_{2}\right)}_{h_{1}-\frac{1}{2}-\rho}, \underbrace{\hat{y}_{(1} \ldots \hat{y}_{1}}_{h_{2}-\frac{1}{2}+\omega} \underbrace{\left.\hat{y}_{2} \ldots \hat{y}_{2}\right)}_{h_{2}-\frac{1}{2}-\omega} K\} \\
& =\mathrm{i} \sum_{r=1}^{\left.\frac{h_{1}+h_{2}}{2}\right]}(-1)^{r}[\mathrm{FF}_{2 r,+}^{h_{1}+\frac{1}{2}, h_{2}+\frac{1}{2}}(\rho, \omega ; \mu) \underbrace{\hat{y}_{(1} \ldots \ldots \ldots \hat{y}_{1}}_{h_{1}+h_{2}-2 r+\rho+\omega} \underbrace{\hat{y}_{2} \ldots \ldots \ldots \hat{y}_{2} \ldots}_{h_{1}+h_{2}-2 r-\rho-\omega} K \\
& -\frac{2\left(h_{1}+h_{2}-r\right)+1}{2 r} \mathrm{FF}_{2 r,-}^{h_{1}+\frac{1}{2}, h_{2}+\frac{1}{2}}(\rho, \omega ; \mu) \underbrace{\hat{y}_{(1 \ldots \ldots} \ldots \ldots . \hat{y}_{1}}_{h_{1}+h_{2}-2 r+\rho+\omega} \underbrace{\hat{y}_{2} \ldots \ldots \ldots \hat{y}_{2)}}_{h_{1}+h_{2}-2 r-\rho-\omega}] .
\end{aligned}
$$

Based on the results of this subsection, we will obtain the $\mathcal{N}=4$ higher spin algebra $s h s_{2}[\mu]$ in next subsection. ${ }^{17}$

\subsection{The (anti)commutators between the $\mathcal{N}=4$ higher spin generators with nonzero $\mu$}

We determine the (anti)commutators of the $\mathcal{N}=4$ higher spin generators by using (4.5), (4.6), (4.7), (4.8), and (4.9).

Let us start with the commutator $\left[\tilde{\Phi}_{2, m}^{\left(s_{1}\right)}, \tilde{\Phi}_{2, n}^{\left(s_{2}\right)}\right]$ in (3.1). The bosonic oscillators do not have the operator $K$ and the $2 \times 2$ identity matrix is included. From the previous observation $[A \otimes I, B \otimes I]=[A, B] \otimes I$ and the realization of higher spin generators in (2.6), we have the following intermediate result for the commutator

$$
\begin{aligned}
{\left[\tilde{\Phi}_{2, m}^{\left(s_{1}\right)}, \tilde{\Phi}_{2, n}^{\left(s_{2}\right)}\right]=} & \left(2 s_{1}-1\right)\left(2 s_{2}-1\right) \\
& \times\left([\underbrace{\hat{y}_{(1} \cdots \hat{y}_{1}}_{s_{1}+1+m} \underbrace{\left.\hat{y}_{2} \cdots \hat{y}_{2}\right)}_{s_{1}+1-m}, \underbrace{\hat{y}_{(1} \cdots \hat{y}_{1}}_{s_{2}+1+n} \underbrace{\left.\hat{y}_{2} \cdots \hat{y}_{2}\right)}_{s_{2}+1-n}]\right) \otimes I_{2 \times 2} .
\end{aligned}
$$

From the relation (4.5) we have obtained, the above commutator (4.10) becomes

$$
\begin{aligned}
& \left(2 s_{1}-1\right)\left(2 s_{2}-1\right) \\
& \times\left(\sum_{r=1}^{\left[\frac{s_{1}+s_{2}+3}{2}\right]} \mathrm{i}(-1)^{r}[\mathrm{BB}_{2 r,+}^{s_{1}+2, s_{2}+2}(m, n ; \mu) \underbrace{\hat{y}_{(1} \ldots \ldots \ldots \hat{y}_{1}}_{s_{1}+s_{2}+3-2 r+m+n} \underbrace{\hat{y}_{2} \ldots \ldots \ldots \hat{y}_{2)}}_{s_{1}+s_{2}+3-2 r-m-n}\right. \\
& -\mathrm{BB}_{2 r,-}^{s_{1}+2, s_{2}+2}(m, n ; \mu) \underbrace{\hat{y}_{(1} \ldots \ldots \ldots \hat{y}_{1}}_{s_{1}+s_{2}+3-2 r+m+n} \underbrace{\hat{y}_{2} \ldots \ldots \ldots \hat{y}_{2)}}_{s_{1}+s_{2}+3-2 r-m-n} K]) \otimes I_{2 \times 2} .
\end{aligned}
$$

\footnotetext{
${ }^{17}$ Let us emphasize again that from the indices of the structure constants we can determine how they appear in the (anti)commutators of oscillators. That is, the sum of upper indices minus lower index provides the spin of the oscillator in the right hand side of (anti)commutators of oscillators. Of course, the upper two indices give the spins of the oscillators in the left hand side of the (anti)commutators respectively. In (4.5), (4.8) and (4.9), there exists only one kind of spin for the oscillator in the right hand side for fixed $r$. Each two structure constants has the same indices for the spins. In (4.6) and (4.7), there are two kinds of spin for the oscillator for fixed $r$. In each case, the lower indices of two structure constants are different from each other.
} 
Because the right hand side contains only $2 \times 2$ identity matrix and the spin is given by $\left(s_{1}+s_{2}+4-2 r\right)$ which is an integer, the only $\mathcal{N}=4$ higher spin generators, $\Phi_{0}^{(s)}$ and $\tilde{\Phi}_{2}^{(s)}$, are allowed. From the relation (2.6), the operator $K$ independent oscillator term in (4.11) can be written as

$$
\underbrace{\hat{y}_{(1} \ldots \ldots \ldots \hat{y}_{1}}_{s_{1}+s_{2}+3-2 r+m+n} \underbrace{\left.\hat{y}_{2} \ldots \ldots \ldots \hat{y}_{2}\right)}_{s_{1}+s_{2}+3-2 r-m-n} \otimes I_{2 \times 2}=-\frac{1}{2\left(s_{1}+s_{2}-2 r+2\right)-1} \tilde{\Phi}_{2, m+n}^{\left(s_{1}+s_{2}-2 r+2\right)} .
$$

Moreover, the operator $K$ dependent term in (4.11) can be described by the following linear combination

$$
\begin{aligned}
& \underbrace{\hat{y}_{(1} \ldots \ldots \ldots \hat{y}_{1}}_{s_{1}+s_{2}+3-2 r+m+n} \underbrace{\hat{y}_{2} \ldots \ldots \ldots \hat{y}_{2)}}_{s_{1}+s_{2}+3-2 r-m-n} K \otimes I_{2 \times 2} \\
= & \frac{1}{2\left(s_{1}+s_{2}-2 r+4\right)-1}\left(\Phi_{0, m+n}^{\left(s_{1}+s_{2}-2 r+4\right)}+\frac{\nu}{2\left(s_{1}+s_{2}-2 r+2\right)-1} \tilde{\Phi}_{2, m+n}^{\left(s_{1}+s_{2}-2 r+2\right)}\right) .
\end{aligned}
$$

Therefore, by combining (4.12) with (4.13), we obtain the final commutator relation which is given by eq. (E.10).

Let us emphasize that in order to apply the three cases (we have described before) for the construction of (anti)commutators between the $\mathcal{N}=4$ higher spin generators, we should include at least one higher spin generator in the left hand side which contains $2 \times 2$ identity matrix. If this is not the case, we should find the relevant (anti)commutators separately. For example, the case $\delta_{i k}\left[\Phi_{1, m}^{(s), i j}, \Phi_{1, n}^{(s), k l}\right]$ described by eq. (E.5) does not have any combination of the commutative matrix. Note that the following commutator between the tensor products provides the tensor product of the anticommutator between the operators and $2 \times 2$ matrix $^{18}$

$$
\left[A \otimes \sigma^{i}, B \otimes \sigma^{j}\right]=\{A, B\} \otimes \mathrm{i} \epsilon^{i j k} \sigma^{k}+[A, B] \otimes \delta^{i j} I_{2 \times 2} .
$$

According to this relation (4.15), we should put the relevant $\mathcal{N}=4$ higher spin generators both sides.

Moreover, it is easy to see that the anticommutators, $\left\{\Phi_{\frac{1}{2}, \rho}^{(s), 1}, \Phi_{\frac{1}{2}, \sigma}^{(s), 1}\right\}$ and $\left\{\Phi_{\frac{1}{2}, \rho}^{(s), 1}, \Phi_{\frac{1}{2}, \sigma}^{(s), 2}\right\}$, cannot be obtained directly by using the relation (4.8) because we have nontrivial structure of Pauli matrices. Instead, we should determine the anticommutators, $\left\{\Phi_{\frac{1}{2}, \rho}^{(s), 4}, \Phi_{\frac{1}{2}, \sigma}^{(s), 4}\right\}$ and $\left\{\Phi_{\frac{1}{2}, \rho}^{(s), 1}, \Phi_{\frac{1}{2}, \sigma}^{(s), 4}\right\}$ first where the $2 \times 2$ identity matrix arises in these anticommutators. Based

$$
\begin{aligned}
& { }^{18} \text { In this case we have } \\
& \{\underbrace{\hat{y}_{(1} \ldots \hat{y}_{1}}_{h_{1}-1+m} \underbrace{\left.\hat{y}_{2} \ldots \hat{y}_{2}\right)}_{h_{1}-1-m}, \underbrace{\hat{y}_{(1} \ldots \hat{y}_{1}}_{h_{2}-1+n} \underbrace{\hat{y}_{2} \ldots \hat{y}_{2)}}_{h_{2}-1-n}\} \\
& =\sum_{r=1}^{\left[\frac{h_{1}+h_{2}}{2}\right]}(-1)^{r}[\mathrm{BB}_{2 r-1,+}^{h_{1}, h_{2}}(m, n ; \mu) \underbrace{\hat{y}_{(1} \ldots \ldots \ldots \hat{y}_{1}}_{h_{1}+h_{2}-2 r+m+n} \underbrace{\left.\hat{y}_{2} \ldots \ldots \ldots \hat{y}_{2}\right)}_{h_{1}+h_{2}-2 r-m-n} \\
& -\mathrm{BB}_{2 r-1,-}^{h_{1}, h_{2}}(m, n ; \mu) \underbrace{\hat{y}_{(1} \ldots \ldots \ldots \hat{y}_{1}}_{h_{1}+h_{2}-2 r+m+n} \underbrace{\hat{y}_{2} \ldots \ldots \ldots \hat{y}_{2}}_{h_{1}+h_{2}-2 r-m-n} K] .
\end{aligned}
$$

Note that there exists an anticommutator rather than commutator in the left side. 
on these anticommutators, we can determine the above anticommutators by keeping track of $\mathrm{SO}(4)$ indices coming from $\mathcal{N}=4$ superspace description.

We present the first five commutators in this section and the remaining ones will be summarized by appendix E. From the case-one (4.5) with the footnote 8, the following commutator is obtained

$$
\begin{aligned}
& {\left[\Phi_{0, m}^{\left(s_{1}\right)}, \Phi_{0, n}^{\left(s_{2}\right)}\right]=} \\
& \sum_{r=1}^{\left[\frac{s_{1}+s_{2}-1}{2}\right]} \mathrm{i}(-1)^{r}\left[\frac { 1 } { 2 ( s _ { 1 } + s _ { 2 } - 2 r ) - 1 } \left(2 \nu\left(s_{1}+s_{2}-1\right) \mathrm{BB}_{2 r,+}^{s_{1}, s_{2}}(m, n ; \mu)\right.\right. \\
& \left.-\left(\left(2 s_{1}-1\right)\left(2 s_{2}-1\right)+\nu^{2}\right) \mathrm{BB}_{2 r,-}^{s_{1}, s_{2}}(m, n ; \mu)\right) \Phi_{0, m+n}^{\left(s_{1}+s_{2}-2 r\right)} \\
& -\frac{1}{\left(2 s_{1}+2 s_{2}-4 r-5\right)\left(2 s_{1}+2 s_{2}-4 r-1\right)}( \\
& \left(\left(2 s_{1}-1\right)\left(2 s_{2}-1\right)\left(2 s_{1}+2 s_{2}-4 r-1\right)+(1-4 r) \nu^{2}\right) \mathrm{BB}_{2 r,+}^{s_{1}, s_{2}}(m, n ; \mu) \\
& \left.+\nu\left(4 s_{2}-4\left(s_{1}^{2}-s_{1}+s_{1} s_{2}+s_{2}^{2}\right)-8 r+8\left(s_{1}+s_{2}\right) r+\nu^{2}-1\right) \mathrm{BB}_{2 r,-}^{s_{1}, s_{2}}(m, n ; \mu)\right) \\
& \left.\times \tilde{\Phi}_{2, m+n}^{\left(s_{1}+s_{2}-2 r-2\right)}\right] .
\end{aligned}
$$

There are two different structure constants having the same indices for fixed $r$.

The following commutator can be determined by using (4.6) and (4.7)

$$
\begin{aligned}
& {\left[\Phi_{0, m}^{\left(s_{1}\right)}, \Phi_{\frac{1}{2}, \rho}^{\left(s_{2}\right), i}\right]=\mathrm{i}\left(2 s_{2}-1\right) \sum_{r=1}^{\left[\frac{s_{1}+s_{2}-1}{2}\right]}\left[(-1)^{r} \frac{1}{2\left(s_{1}+s_{2}-2 r\right)-1}\right.} \\
& \left.\left(\nu \mathrm{BF}_{2 r,+}^{s_{1}, s_{2}+\frac{1}{2}}(m, \rho ; \mu)+\frac{\left(2 s_{1}-1\right)\left(2 s_{1}-2 r-1\right)}{2 r} \mathrm{BF}_{2 r,-}^{s_{1}, s_{2}+\frac{1}{2}}(m, \rho ; \mu)\right) \Phi_{\frac{1}{2}, m+\rho}^{\left(s_{1}+s_{2}-2 r\right), i}\right] \\
& -\mathrm{i}\left(2 s_{2}-1\right) \sum_{r=1}^{\left[\frac{s_{1}+s_{2}}{2}\right]}\left[(-1)^{r} \frac{1}{2\left(s_{1}+s_{2}-2 r\right)-1}\right. \\
& \left.\left(\left(2 s_{1}-1\right) \mathrm{BF}_{2 r-1,+}^{s_{1}, s_{2}+\frac{1}{2}}(m, \rho ; \mu)+\frac{2 \nu\left(s_{1}-r\right)}{(2 r-1)} \mathrm{BF}_{2 r-1,-}^{s_{1}, s_{2}+\frac{1}{2}}(m, \rho ; \mu)\right) \tilde{\Phi}_{\frac{3}{2}, m+\rho}^{\left(s_{1}+s_{2}-2 r\right), i}\right] .
\end{aligned}
$$

There are four different structure constants where each two of them have same lower indices for fixed $r$.

As done for (4.16), we obtain the following commutator

$$
\begin{aligned}
& {\left[\Phi_{0, m}^{\left(s_{1}\right)}, \Phi_{1, n}^{\left(s_{2}\right), i j}\right]=-\sum_{r=1}^{\left[\frac{s_{1}+s_{2}}{2}\right]} \frac{\mathrm{i}(-1)^{r}\left(2 s_{2}-1\right)}{2\left(s_{1}+s_{2}-2 r\right)-1}[} \\
& \quad\left(\left(2 s_{1}-1\right) \mathrm{BB}_{2 r,-}^{s_{1}, s_{2}+1}(m, n ; \mu)-\nu \mathrm{BB}_{2 r,+}^{s_{1}, s_{2}+1}(m, n ; \mu)\right) \Phi_{1, m+n}^{\left(s_{1}+s_{2}-2 r\right), i j} \\
& \left.+\left(\left(2 s_{1}-1\right) \mathrm{BB}_{2 r,+}^{s_{1}, s_{2}+1}(m, n ; \mu)-\nu \mathrm{BB}_{2 r,-}^{s_{1}, s_{2}+1}(m, n ; \mu)\right) \Phi_{1, m+n}^{\left(s_{1}+s_{2}-2 r\right), i j}\right] .
\end{aligned}
$$

The two different structure constants have the same indices for fixed $r$. 
From the analysis of footnote 9 and (4.7), the following commutator satisfies

$$
\begin{aligned}
& {\left[\Phi_{0, m}^{\left(s_{1}\right)}, \tilde{\Phi}_{\frac{3}{2}, \rho}^{\left(s_{2}\right), i}\right]=\sum_{r=1}^{\left[\frac{s_{1}+s_{2}+1}{2}\right]}\left[\mathrm{i}(-1)^{r}\left(2 s_{2}-1\right) \frac{1}{2\left(s_{1}+s_{2}-2 r\right)+3}\right.} \\
& \left.\left(\left(2 s_{1}-1\right) \mathrm{BF}_{2 r-1,+}^{s_{1}, s_{2}+\frac{3}{2}}(m, \rho ; \mu)+\frac{2 \nu\left(s_{1}-r\right)}{(2 r-1)} \mathrm{BF}_{2 r-1,-}^{s_{1}, s_{2}+\frac{3}{2}}(m, \rho ; \mu)\right) \Phi_{\frac{1}{2}, m+\rho}^{\left(s_{1}+s_{2}-2 r+2\right), i}\right] \\
& +\sum_{r=1}^{\left[\frac{s_{1}+s_{2}}{2}\right]}\left[\mathrm{i}(-1)^{r}\left(2 s_{2}-1\right) \frac{1}{2\left(s_{1}+s_{2}-2 r\right)-1}\right. \\
& \left.\left(\nu \mathrm{BF}_{2 r,+}^{s_{1}, s_{2}+\frac{3}{2}}(m, \rho ; \mu)+\frac{\left(2 s_{1}-1\right)\left(2 s_{1}-2 r-1\right)}{2 r} \mathrm{BF}_{2 r,-}^{s_{1}, s_{2}+\frac{3}{2}}(m, \rho ; \mu)\right) \tilde{\Phi}_{\frac{3}{2}, m+\rho}^{\left(s_{1}+s_{2}-2 r\right), i}\right]
\end{aligned}
$$

Two of the structure constants have the lower indices $(2 r-1)$ and $2 r$ respectively for fixed $r$.

Finally, we have the following commutator relation

$$
\begin{aligned}
& {\left[\Phi_{0, m}^{\left(s_{1}\right)}, \tilde{\Phi}_{2, n}^{\left(s_{2}\right)}\right]=\mathrm{i}\left(2 s_{2}-1\right)\left[\sum_{r=1}^{\left[\frac{s_{1}+s_{2}+1}{2}\right]} \frac{(-1)^{r}}{2\left(s_{1}+s_{2}-2 r\right)+3}(\right.} \\
& \left.\nu \mathrm{BB}_{2 r,-}^{s_{1}, s_{2}+2}(m, n ; \mu)-\left(2 s_{1}-1\right) \mathrm{BB}_{2 r,+}^{s_{1}, s_{2}+2}(m, n ; \mu)\right) \Phi_{0, m+n}^{\left(s_{1}+s_{2}-2 r+2\right)} \\
& +\sum_{r=1}^{\left[\frac{s_{1}+s_{2}}{2}\right]} \frac{(-1)^{r}}{2\left(s_{1}+s_{2}-2 r\right)+3} \frac{1}{\left(2 s_{1}+2 s_{2}-4 r-1\right)}\left(2 \nu\left(s_{2}-2 r+2\right) \mathrm{BB}_{2 r,+}^{s_{1}, s_{2}+2}(m, n ; \mu)\right. \\
& \left.\left.-\left(\left(2 s_{1}-1\right)\left(2 s_{1}+2 s_{2}-4 r+3\right)-\nu^{2}\right) \mathrm{BB}_{2 r,-}^{s_{1}, s_{2}+2}(m, n ; \mu)\right) \tilde{\Phi}_{2, m+n}^{\left(s_{1}+s_{2}-2 r\right)}\right] .
\end{aligned}
$$

Again the two different structure constants have the same indices for fixed $r$.

Therefore, the five commutators, (4.16), (4.17), (4.18), (4.19) and (4.20) with common higher spin- $s_{1}$ generator $\Phi_{0, m}^{\left(s_{1}\right)}$, where the $2 \times 2$ identity matrix arises in the left hand side, are determined. The remaining ten (anti)commutators are presented in appendix E.

\section{Conclusions and outlook}

We have obtained the $\mathcal{N}=4$ higher spin algebra $s h s_{2}[\mu]$ which is given by (4.16)-(4.20) and eqs. (E.1)-(E.10) for generic $\mu$. The structure constants are given in (4.2). As a subalgebra, the $\mathcal{N}=2$ higher spin algebra $\operatorname{shs}[\mu]$ arises.

So far, we have considered $2 \times 2$ matrix generalization of $A d S_{3}$ Vasiliev higher spin theory. It is natural to ask about its $M \times M$ matrix generalization where $M \geq 3$. According to [1], the field contents are given by $\left(2 M^{2}-1\right)$ spin 1 fields and $2 M^{2}$ fields of spin $s=\frac{3}{2}, 2, \frac{5}{2}, 3, \cdots$. Although it is known that there is no supersymmetry, it would be interesting to understand the higher spin algebra $h s_{M}[\mu]$ and its nonlinear $\mathcal{W}_{\infty}^{M}[l, \mu]$ algebra where $l$ is the level of $\mathrm{SU}(M)$ currents [46].

Furthermore, it is an open problem to observe any relations between this higher spin algebra $h s_{M}[\mu]$ and the rectangular $W$ algebra studied in [45-47]. In the higher spin 
square [60,61] or in the two dimensional SYK model [62], there exists an additional parameter, like as the above $M$, in the theory. It would be interesting to understand the role of this additional parameter in the various different models.

Other type of matrix generalization of $A d S_{3}$ Vasiliev higher spin theory can be seen from the work of [63] and corresponding coset model is studied in [64] further. It would be interesting to obtain the $\mathcal{N}=3$ higher spin algebra explicitly. Finally, we can apply the present method to the $\mathcal{N}=4$ orthogonal coset model $[65,66]$ and want to obtain the corresponding $\mathcal{N}=4$ higher spin algebra [12, 47].

\section{Acknowledgments}

MHK was supported by an appointment to the YST Program at the APCTP through the Science and Technology Promotion Fund and Lottery Fund of the Korean Government. MHK was also supported by the Korean Local Governments - Gyeongsangbuk-do Province and Pohang City. This work of CA was supported by the National Research Foundation of Korea(NRF) grant funded by the Korea government(MSIT)(No. 2020R1F1A1066893). CA acknowledges warm hospitality from the School of Liberal Arts (and Institute of Convergence Fundamental Studies), Seoul National University of Science and Technology.

\section{A Structure constants for vanishing $\mu$}

The structure constants in subsection 3.1 are given by [11]

$$
\begin{aligned}
p_{\mathrm{F}, h}^{h_{1} h_{2}}(m, n)= & \frac{1}{2(h+1) !} \phi_{h}^{h_{1}, h_{2}}\left(0,-\frac{1}{2}\right) N_{h}^{h_{1}, h_{2}}(m, n), \\
p_{\mathrm{B}, h}^{h_{1} h_{2}}(m, n)= & \frac{1}{2(h+1) !} \phi_{h}^{h_{1}, h_{2}}(0,0) N_{h}^{h_{1}, h_{2}}(m, n), \\
q_{\mathrm{F}, h}^{h_{1} h_{2}}(m, r)= & \frac{(-1)^{h}}{4(h+2) !}\left(\left(h_{1}-1\right) \phi_{h+1}^{h_{1}, h_{2}+\frac{1}{2}}(0,0)\right. \\
& \left.-\left(h_{1}-h-3\right) \phi_{h+1}^{h_{1}, h_{2}+\frac{1}{2}}\left(0,-\frac{1}{2}\right)\right) N_{h}^{h_{1}, h_{2}}(m, n), \\
q_{\mathrm{B}, h}^{h_{1} h_{2}}(m, r)= & \frac{-1}{4(h+2) !}\left(\left(h_{1}-h-2\right) \phi_{h+1}^{h_{1}, h_{2}+\frac{1}{2}}(0,0)\right. \\
& \left.-\left(h_{1}\right) \phi_{h+1}^{h_{1}, h_{2}+\frac{1}{2}}\left(0,-\frac{1}{2}\right)\right) N_{h}^{h_{1}, h_{2}}(m, n), \\
& \frac{4(-1)^{h}}{h !}\left(\left(h_{1}+h_{2}-1-h\right) \phi_{h}^{h_{1}+\frac{1}{2}, h_{2}+\frac{1}{2}}\left(\frac{1}{2},-\frac{1}{4}\right)\right. \\
& \left.-\left(h_{1}+h_{2}-\frac{3}{2}-h\right) \phi_{h+1}^{h_{1}+\frac{1}{2}, h_{2}+\frac{1}{2}}\left(\frac{1}{2},-\frac{1}{4}\right)\right) N_{h-1}^{h_{1}, h_{2}}(m, n), \\
o_{\mathrm{F}, h}^{h_{1} h_{2}}(r, s)= & \frac{4}{h !}\left(\left(h_{1}+h_{2}-2-h\right) \phi_{h}^{h_{1}+\frac{1}{2}, h_{2}+\frac{1}{2}}\left(\frac{1}{2},-\frac{1}{4}\right)\right. \\
& \left.-\left(h_{1}+h_{2}-\frac{3}{2}-h\right) \phi_{h+1}^{h_{1}+\frac{1}{2}, h_{2}+\frac{1}{2}}\left(\frac{1}{2},-\frac{1}{4}\right)\right) N_{h-1}^{h_{1}, h_{2}}(m, n), \\
o_{\mathrm{B}, h}^{h_{1} h_{2}}(r, s), &
\end{aligned}
$$


where the mode dependent function in eq. (A.1) and the symbol $\phi_{h}^{h_{1}, h_{2}}(x, y)$ are given by

$$
\begin{aligned}
N_{h}^{h_{1}, h_{2}}(m, n)= & \sum_{l=0}^{h+1}(-1)^{l}\left(\begin{array}{c}
h+1 \\
l
\end{array}\right)\left[h_{1}-1+m\right]_{h+1-l}\left[h_{1}-1-m\right]_{l} \\
& \times\left[h_{2}-1+n\right]_{l}\left[h_{2}-1-n\right]_{h+1-l}, \\
\phi_{h}^{h_{1}, h_{2}}(x, y)= & { }_{4} F_{3}\left[\begin{array}{c}
-\frac{1}{2}-x-2 y, \frac{3}{2}-x+2 y,-\frac{h+1}{2}+x,-\frac{h}{2}+x \\
-h_{1}+\frac{3}{2},-h_{2}+\frac{3}{2}, h_{1}+h_{2}-h-\frac{3}{2}
\end{array} ; 1 .\right.
\end{aligned}
$$

In eq. (A.2), we introduce the generalized hypergeometric function as follows:

$$
{ }_{4} F_{3}\left[\begin{array}{c}
a_{1}, a_{2}, a_{3}, a_{4} \\
b_{1}, b_{2}, b_{3}
\end{array} ; z\right]=\sum_{n=0}^{\infty} \frac{\left(a_{1}\right)_{n}\left(a_{2}\right)_{n}\left(a_{3}\right)_{n}\left(a_{4}\right)_{n}}{\left(b_{1}\right)_{n}\left(b_{2}\right)_{n}\left(b_{3}\right)_{n}} \frac{z^{n}}{n !} .
$$

The falling and rising Pochhammer symbols in eq. (A.2) and eq. (A.3) $[a]_{n}$ and $(a)_{n}$ are defined as $[a]_{n} \equiv a(a-1) \cdots(a-n+1)$ and $(a)_{n} \equiv a(a+1) \cdots(a+n-1)$.

\section{B The (anti)commutators between the oscillators with vanishing $\mu$ in Odake basis}

We present here the (anti)commutators between the oscillators with vanishing $\mu$ in Odake basis where the structure constants are given in terms of generalized hypergeometric functions.

- The case-one with two bosonic oscillators. We have

$$
\begin{aligned}
& {[\underbrace{\hat{y}_{(1} \ldots \hat{y}_{1}}_{h_{1}-1+m} \underbrace{\hat{y}_{2} \ldots \hat{y}_{2)}}_{h_{1}-1-m}, \underbrace{\hat{y}_{(1} \ldots \hat{y}_{1}}_{h_{2}-1+n} \underbrace{\left.\hat{y}_{2} \ldots \hat{y}_{2}\right)}_{h_{2}-1-n}]=}
\end{aligned}
$$

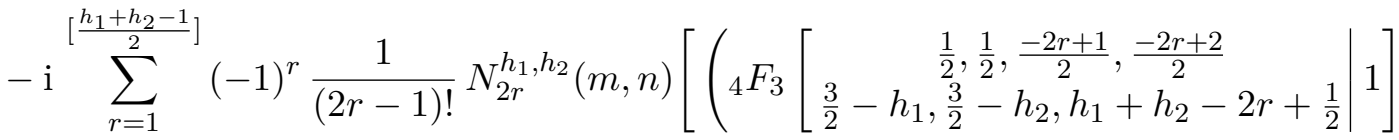

$$
\begin{aligned}
& \left.+{ }_{4} F_{3}\left[\begin{array}{c}
\frac{3}{2},-\frac{1}{2}, \frac{-2 r+1}{2}, \frac{-2 r+2}{2} \\
\frac{3}{2}-h_{1}, \frac{3}{2}-h_{2}, h_{1}+h_{2}-2 r+\frac{1}{2}
\end{array} \mid 1\right]\right) \underbrace{\hat{y}_{(1} \ldots \ldots \ldots \ldots \hat{y}_{1}}_{h_{1}+h_{2}-2 r-1+m+n} \underbrace{\left.\hat{y}_{2} \ldots \ldots \ldots \ldots \hat{y}_{2}\right)}_{h_{1}+h_{2}-2 r-1-m-n} \\
& -\left({ }_{4} F_{3}\left[\begin{array}{c|c}
\frac{1}{2}, \frac{1}{2}, \frac{-2 r+1}{2}, \frac{-2 r+2}{2} \\
\frac{3}{2}-h_{1}, \frac{3}{2}-h_{2}, h_{1}+h_{2}-2 r+\frac{1}{2}
\end{array} \mid 1\right]\right. \\
& \left.-{ }_{4} F_{3}\left[\begin{array}{c|c}
\frac{3}{2},-\frac{1}{2}, \frac{-2 r+1}{2}, \frac{-2 r+2}{2} \\
\frac{3}{2}-h_{1}, \frac{3}{2}-h_{2}, h_{1}+h_{2}-2 r+\frac{1}{2} \mid
\end{array} \mid 1\right]\right) \\
& \times \underbrace{\hat{y}_{(1} \ldots \ldots \ldots \ldots \hat{y}_{1}}_{h_{1}+h_{2}-2 r-1+m+n} \underbrace{\hat{y}_{2} \ldots \ldots \ldots \ldots \hat{y}_{2)}}_{h_{1}+h_{2}-2 r-1-m-n} K] \text {. }
\end{aligned}
$$

The above commutation relation eq. (B.1) is the same as the one in (3.4). Moreover, this commutation relation eq. (B.1) can be obtained by taking $\mu$ to zero in eq. (D.1). 
- The case-two with one bosonic and one fermionic oscillators. By substituting the corresponding structure constants, we obtain

$$
\begin{aligned}
& {[\underbrace{\hat{y}_{(1} \ldots \hat{y}_{1}}_{h_{1}-1+m} \underbrace{\hat{y}_{2} \ldots \hat{y}_{2)}}_{h_{1}-1-m}, \underbrace{\hat{y}_{(1} \ldots \hat{y}_{1}}_{h_{2}-\frac{1}{2}+\rho} \underbrace{\left.\hat{y}_{2} \ldots \hat{y}_{2}\right)}_{h_{2}-\frac{1}{2}-\rho}]=} \\
& -\sum_{r=1}^{\left[\frac{h_{1}+h_{2}-1}{2}\right]}\left[\frac { \mathrm { i } ( - 1 ) ^ { r } } { ( 2 r ) ! } N _ { 2 r } ^ { h _ { 1 } , h _ { 2 } + \frac { 1 } { 2 } } ( m , \rho ) \left((2 r+1)_{4} F_{3}\left[\begin{array}{c}
\frac{1}{2}, \frac{1}{2}, \frac{-2 r}{2}, \frac{-2 r+1}{2} \\
\frac{3}{2}-h_{1}, \frac{1}{2}-h_{2}, h_{1}+h_{2}-2 r+\frac{1}{2}
\end{array} \mid 1\right]\right.\right. \\
& +(2 r-1){ }_{4} F_{3}\left[\begin{array}{c}
\frac{3}{2},-\frac{1}{2}, \frac{-2 r}{2}, \frac{-2 r+1}{2} \\
\frac{3}{2}-h_{1}, \frac{1}{2}-h_{2}, h_{1}+h_{2}-2 r+\frac{1}{2} \mid 1
\end{array} \mid\right) \\
& \times \underbrace{\hat{y}_{(1} \ldots \ldots \ldots \hat{y}_{1}}_{h_{1}+h_{2}-2 r-\frac{1}{2}+m+\rho} \underbrace{\hat{y}_{2} \ldots \ldots \ldots \ldots \hat{y}_{2}}_{h_{1}+h_{2}-2 r-\frac{1}{2}-m-\rho}] \\
& +\sum_{r=1}^{\left[\frac{h_{1}+h_{2}}{2}\right]}\left[(-1)^{r} \frac{2\left(h_{1}-r\right)}{(2 r-1) !} N_{2 r-1}^{h_{1}, h_{2}+\frac{1}{2}}(m, \rho) \times\left({ }_{4} F_{3}\left[\begin{array}{c}
\frac{1}{2}, \frac{1}{2}, \frac{-2 r+1}{2}, \frac{-2 r+2}{2} \\
\frac{3}{2}-h_{1}, \frac{1}{2}-h_{2}, h_{1}+h_{2}-2 r+\frac{3}{2}
\end{array} \mid 1\right]\right.\right. \\
& \left.-{ }_{4} F_{3}\left[\begin{array}{c|c}
\frac{3}{2},-\frac{1}{2}, \frac{-2 r+1}{2}, \frac{-2 r+2}{2} \\
\frac{3}{2}-h_{1}, \frac{1}{2}-h_{2}, h_{1}+h_{2}-2 r+\frac{3}{2}
\end{array} \mid 1\right]\right) \\
& \times \underbrace{\hat{y}_{(1} \ldots \ldots \ldots \ldots \hat{y}_{1}}_{h_{1}+h_{2}-2 r+\frac{1}{2}+m+\rho} \underbrace{\hat{y}_{2} \ldots \ldots \ldots \ldots \hat{y}_{2)}}_{h_{1}+h_{2}-2 r+\frac{1}{2}-m-\rho} K] \text {. }
\end{aligned}
$$

This is equivalent to (3.5) and we observe that this is generalized to eq. (D.2).

Similarly, we have

$$
\begin{aligned}
& {[\underbrace{\hat{y}_{(1} \ldots \hat{y}_{1}}_{h_{1}-1+m} \underbrace{\left.\hat{y}_{2} \ldots \hat{y}_{2}\right)}_{h_{1}-1-m} K, \underbrace{\hat{y}_{(1} \ldots \hat{y}_{1}}_{h_{2}-\frac{1}{2}+\rho} \underbrace{\hat{y}_{2} \ldots \hat{y}_{2)}}_{h_{2}-\frac{1}{2}-\rho}]=} \\
& \sum_{r=1}^{\left[\frac{h_{1}+h_{2}}{2}\right]}\left[\frac { ( - 1 ) ^ { r } } { ( 2 r - 1 ) ! } N _ { 2 r - 1 } ^ { h _ { 1 } , h _ { 2 } + \frac { 1 } { 2 } } ( m , \rho ) \left((2 r)_{4} F_{3}\left[\begin{array}{c}
\frac{1}{2}, \frac{1}{2}, \frac{-2 r+1}{2}, \frac{-2 r+2}{2} \\
\frac{3}{2}-h_{1}, \frac{1}{2}-h_{2}, h_{1}+h_{2}-2 r+\frac{3}{2}
\end{array} \mid 1\right]\right.\right. \\
& \left.+(2 r-2)_{4} F_{3}\left[\begin{array}{c|c}
\frac{3}{2},-\frac{1}{2}, \frac{-2 r+1}{2}, \frac{-2 r+2}{2} \\
\frac{3}{2}-h_{1}, \frac{1}{2}-h_{2}, h_{1}+h_{2}-2 r+\frac{3}{2}
\end{array} \mid 1\right]\right) \\
& \times \underbrace{\hat{y}_{(1} \ldots \ldots \ldots \ldots \hat{y}_{1}}_{h_{1}+h_{2}-2 r+\frac{1}{2}+m+\rho} \underbrace{\left.\hat{y}_{2} \ldots \ldots \ldots \ldots . \hat{y}_{2}\right)}_{h_{1}+h_{2}-2 r+\frac{1}{2}-m-\rho} K] \\
& -\mathrm{i} \sum_{r=1}^{\left[\frac{h_{1}+h_{2}-1}{2}\right]}\left[( - 1 ) ^ { r } \frac { 2 ( h _ { 1 } - r - \frac { 1 } { 2 } ) } { ( 2 r ) ! } N _ { 2 r } ^ { h _ { 1 } , h _ { 2 } + \frac { 1 } { 2 } } ( m , \rho ) \left({ }_{4} F_{3}\left[\begin{array}{c}
\frac{1}{2}, \frac{1}{2}, \frac{-2 r}{2}, \frac{-2 r+1}{2} \\
\frac{3}{2}-h_{1}, \frac{1}{2}-h_{2}, h_{1}+h_{2}-2 r+\frac{1}{2}
\end{array} \mid 1\right]\right.\right.
\end{aligned}
$$

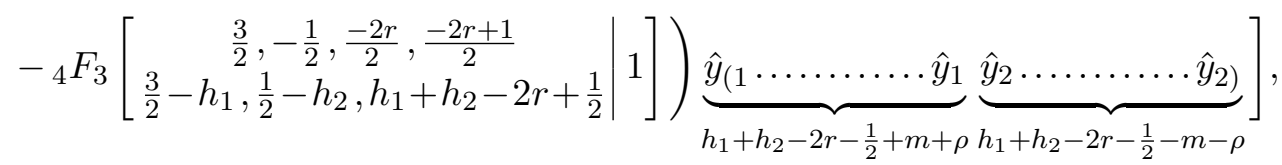

which is equivalent to (3.6) and is generalized to eq. (D.9). 
- The case-three with two fermionic oscillators. The next case can be written as

$$
\begin{aligned}
& \{\underbrace{\hat{y}_{(1} \ldots \hat{y}_{1}}_{h_{1}-\frac{1}{2}+\rho} \underbrace{\left.\hat{y}_{2} \ldots \hat{y}_{2}\right)}_{h_{1}-\frac{1}{2}-\rho}, \underbrace{\hat{y}_{(1} \ldots \hat{y}_{1}}_{h_{2}-\frac{1}{2}+\omega} \underbrace{\hat{y}_{2} \ldots \hat{y}_{2)}}_{h_{2}-\frac{1}{2}-\omega}\}= \\
& -2 \sum_{r=1}^{\left[\frac{h_{1}+h_{2}+1}{2}\right]}(-1)^{r} \frac{1}{(2 r-2) !} N_{2 r-1}^{h_{1}+\frac{1}{2}, h_{2}+\frac{1}{2}}(\rho, \omega)[ \\
& \times\left({ }_{4} F_{3}\left[\begin{array}{c}
\frac{1}{2},-\frac{1}{2}, \frac{-2 r+2}{2}, \frac{-2 r+3}{2} \\
\frac{1}{2}-h_{1}, \frac{1}{2}-h_{2}, h_{1}+h_{2}-2 r+\frac{5}{2}
\end{array} \mid 1\right]\right) \underbrace{\hat{y}_{(1} \ldots \ldots \ldots \hat{y}_{1}}_{h_{1}+h_{2}-2 r+1+\rho+\omega} \underbrace{\left.\hat{y}_{2} \ldots \ldots \ldots \hat{y}_{2}\right)}_{h_{1}+h_{2}-2 r+1-\rho-\omega} \\
& +\left(2 h_{1}+2 h_{2}-4 r+3\right)\left({ }_{4} F_{3}\left[\begin{array}{c|c}
\frac{1}{2},-\frac{1}{2}, \frac{-2 r+1}{2}, \frac{-2 r+2}{2} \\
\frac{1}{2}-h_{1}, \frac{1}{2}-h_{2}, h_{1}+h_{2}-2 r+\frac{3}{2}
\end{array} \mid 1\right]\right. \\
& \left.-{ }_{4} F_{3}\left[\begin{array}{c}
\frac{1}{2},-\frac{1}{2}, \frac{-2 r+2}{2}, \frac{-2 r+3}{2} \\
\frac{1}{2}-h_{1}, \frac{1}{2}-h_{2}, h_{1}+h_{2}-2 r+\frac{5}{2}
\end{array} \mid 1\right]\right) \underbrace{\hat{y}_{(1} \ldots \ldots \ldots \hat{y}_{1}}_{h_{1}+h_{2}-2 r+1+\rho+\omega} \underbrace{\left.\hat{y}_{2} \ldots \ldots \ldots \hat{y}_{2}\right)}_{h_{1}+h_{2}-2 r+1-\rho-\omega} K] \text {, }
\end{aligned}
$$

which is equivalent to (3.7) and we observe that this is generalized to eq. (D.10). Note that by substituting the structure constants appearing in appendix A into the ones in (3.7), the second $\phi$ in $o_{F}$ and $o_{B}$ has different sign leading to the one generalized hypergeometric function (not two).

Finally, the following relation holds

$$
\begin{aligned}
& \{\underbrace{\hat{y}_{(1} \ldots \hat{y}_{1}}_{h_{1}-\frac{1}{2}+\rho} \underbrace{\hat{y}_{2} \ldots \hat{y}_{2}}_{h_{1}-\frac{1}{2}-\rho}, \underbrace{\hat{y}_{(1} \ldots \hat{y}_{1}}_{h_{2}-\frac{1}{2}+\omega} \underbrace{\left.\hat{y}_{2} \ldots \hat{y}_{2}\right)}_{h_{2}-\frac{1}{2}-\omega} K\}= \\
& -2 \mathrm{i} \sum_{r=1}^{\left[\frac{h_{1}+h_{2}}{2}\right]}(-1)^{r} \frac{1}{(2 r-1) !} N_{2 r}^{h_{1}+\frac{1}{2}, h_{2}+\frac{1}{2}}(\rho, \omega)[ \\
& \times\left({ }_{4} F_{3}\left[\begin{array}{c|c}
\frac{1}{2},-\frac{1}{2}, \frac{-2 r+1}{2}, \frac{-2 r+2}{2} \\
\frac{1}{2}-h_{1}, \frac{1}{2}-h_{2}, h_{1}+h_{2}-2 r+\frac{3}{2}
\end{array} \mid 1\right]\right) \underbrace{\hat{y}_{\left(1 \ldots \ldots . \hat{y}_{1}\right.}}_{h_{1}+h_{2}-2 r+\rho+\omega} \underbrace{\left.\hat{y}_{2} \ldots \ldots \ldots \hat{y}_{2}\right)}_{h_{1}+h_{2}-2 r-\rho-\omega} K \\
& +\left(2 h_{1}+2 h_{2}-4 r+1\right)\left({ }_{4} F_{3}\left[\begin{array}{c|c}
\frac{1}{2},-\frac{1}{2}, \frac{-2 r}{2}, \frac{-2 r+1}{2} \\
\frac{1}{2}-h_{1}, \frac{1}{2}-h_{2}, h_{1}+h_{2}-2 r+\frac{1}{2}
\end{array} \mid 1\right]\right.
\end{aligned}
$$

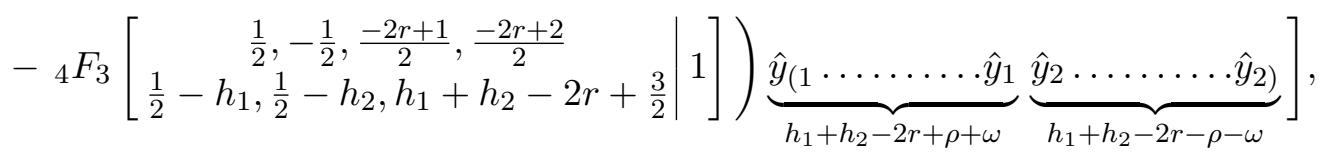

which is equivalent to (3.8) and in eq. (D.13) its generalization is given. 


\section{C $\mu$-dependence in the (anti)commutators of the oscillators up to a total spin $11\left(h_{1}+h_{2} \leq 11\right)$}

\section{C.1 The case-two with one bosonic and one fermionic oscillators}

\section{C.1.1 No operator $K$ dependence in the oscillators}

We continue to analyze the procedure in section 3. As done in (3.10), from (3.4), we introduce the $\mu$ dependent structure constant as follows:

$$
\begin{aligned}
& {[\underbrace{\hat{y}_{(1} \ldots \hat{y}_{1}}_{h_{1}-1+m} \underbrace{\left.\hat{y}_{2} \ldots \hat{y}_{2}\right)}_{h_{1}-1-m}, \underbrace{\hat{y}_{(1} \ldots \hat{y}_{1}}_{h_{2}-\frac{1}{2}+\rho} \underbrace{\hat{y}_{2} \ldots \hat{y}_{2)}}_{h_{2}-\frac{1}{2}-\rho}]=} \\
& \sum_{r=1}^{\left[\frac{h_{1}+h_{2}-1}{2}\right]}(-1)^{r}[N_{2 r}^{h_{1} h_{2}+\frac{1}{2}}(m, \rho) \frac{(-\mathrm{i})}{(2 r-1) !} a_{2 r}^{h_{1}, h_{2}+\frac{1}{2}}(\nu) \underbrace{\hat{y}_{(1} \ldots \ldots \ldots \ldots \hat{y}_{1}}_{h_{1}+h_{2}-2 r-\frac{1}{2}+m+\rho} \underbrace{\left.\hat{y}_{2} \ldots \ldots \ldots \ldots \hat{y}_{2}\right)}_{h_{1}+h_{2}-2 r-\frac{1}{2}-m-\rho}] \\
& +\sum_{r=1}^{\left[\frac{h_{1}+h_{2}}{2}\right]}(-1)^{r}[N_{2 r-1}^{h_{1} h_{2}+\frac{1}{2}}(m, \rho) \frac{2\left(h_{1}-r\right)}{(2 r-1) !} b_{2 r-1}^{h_{1}, h_{2}+\frac{1}{2}}(\nu) \underbrace{\hat{y}_{(1} \ldots \ldots \ldots \ldots \hat{y}_{1}}_{h_{1}+h_{2}-2 r+\frac{1}{2}+m+\rho} \underbrace{\hat{y}_{2} \ldots \ldots \ldots \ldots \hat{y}_{2)}}_{h_{1}+h_{2}-2 r+\frac{1}{2}-m-\rho} K] .
\end{aligned}
$$

By starting with the spins $\left(h_{1}, h_{2}\right)=(2,1)$ and varying the spins, we can compute the commutators for several cases. Then it turns out that the structure constants associated with the oscillators (and without the operator $K$ in eq. (C.1)) can be determined as follows:

$$
\begin{aligned}
& a_{2}^{2, \frac{3}{2}}=2, \quad a_{2}^{2, \frac{5}{2}}=2, \quad a_{2}^{2, \frac{7}{2}}=2, \quad a_{2}^{2, \frac{9}{2}}=2, \quad a_{2}^{2, \frac{11}{2}}=2, \quad a_{2}^{2, \frac{13}{2}}=2, \\
& a_{2}^{2, \frac{15}{2}}=2, \quad a_{2}^{2, \frac{17}{2}}=2, \quad a_{2}^{3, \frac{3}{2}}=2, \quad a_{2}^{3, \frac{5}{2}}=2, a_{4}^{3, \frac{5}{2}}=-\frac{2}{9}(-3+\nu)(3+\nu), \\
& a_{2}^{3, \frac{7}{2}}=2, \quad a_{4}^{3, \frac{7}{2}}=-\frac{2}{25}(-5+\nu)(5+\nu), \quad a_{2}^{3, \frac{9}{2}}=2, \\
& a_{4}^{3, \frac{9}{2}}=-\frac{2}{49}(-7+\nu)(7+\nu), \quad a_{2}^{3, \frac{11}{2}}=2, \quad a_{4}^{3, \frac{11}{2}}=-\frac{2}{81}(-9+\nu)(9+\nu), \\
& a_{2}^{3, \frac{13}{2}}=2, \quad a_{4}^{3, \frac{13}{2}}=-\frac{2}{121}(-11+\nu)(11+\nu), \quad a_{2}^{3, \frac{15}{2}}=2, \\
& a_{4}^{3, \frac{15}{2}}=-\frac{2}{169}(-13+\nu)(13+\nu), \quad a_{2}^{4, \frac{3}{2}}=2, \quad a_{2}^{4, \frac{5}{2}}=2, \\
& a_{4}^{4, \frac{5}{2}}=-\frac{2}{25}(-5+\nu)(5+\nu), \quad a_{2}^{4, \frac{7}{2}}=2, \quad a_{4}^{4, \frac{7}{2}}=-\frac{2}{175}\left(-175+3 \nu^{2}\right), \\
& a_{6}^{4, \frac{7}{2}}=\frac{2}{225}(-5+\nu)(-3+\nu)(3+\nu)(5+\nu), \\
& a_{2}^{4, \frac{9}{2}}=2, a_{4}^{4, \frac{9}{2}}=-\frac{2}{105}\left(-105+\nu^{2}\right), a_{6}^{4, \frac{9}{2}}=\frac{2}{1225}(-7+\nu)(-5+\nu)(5+\nu)(7+\nu), \\
& a_{2}^{4, \frac{11}{2}}=2, \quad a_{4}^{4, \frac{11}{2}}=-\frac{2}{165}\left(-165+\nu^{2}\right), \\
& a_{6}^{4, \frac{11}{2}}=\frac{2}{3969}(-9+\nu)(-7+\nu)(7+\nu)(9+\nu), \quad a_{2}^{4, \frac{13}{2}}=2, \\
& a_{4}^{4, \frac{13}{2}}=-\frac{2}{715}\left(-715+3 \nu^{2}\right), \quad a_{6}^{4, \frac{13}{2}}=\frac{2}{9801}(-11+\nu)(-9+\nu)(9+\nu)(11+\nu), \\
& a_{2}^{5, \frac{3}{2}}=2, \quad a_{2}^{5, \frac{5}{2}}=2, \quad a_{4}^{5, \frac{5}{2}}=-\frac{2}{49}(-7+\nu)(7+\nu), \\
& a_{2}^{5, \frac{7}{2}}=2, a_{4}^{5, \frac{7}{2}}=-\frac{2}{105}\left(-105+\nu^{2}\right), a_{6}^{5, \frac{7}{2}}=\frac{2}{1225}(-7+\nu)(-5+\nu)(5+\nu)(7+\nu),
\end{aligned}
$$




$$
\begin{aligned}
& a_{2}^{5, \frac{9}{2}}=2, \quad a_{4}^{5, \frac{9}{2}}=-\frac{2}{539}\left(-539+3 \nu^{2}\right), a_{6}^{5, \frac{9}{2}}=\frac{2}{5145}(-7+\nu)(7+\nu)\left(-105+\nu^{2}\right), \\
& a_{8}^{5, \frac{9}{2}}=-\frac{2}{11025}(-7+\nu)(-5+\nu)(-3+\nu)(3+\nu)(5+\nu)(7+\nu), \quad a_{2}^{5, \frac{11}{2}}=2, \\
& a_{4}^{5, \frac{11}{2}}=-\frac{2}{273}\left(-273+\nu^{2}\right), \quad a_{6}^{5, \frac{11}{2}}=\frac{2}{43659}(-9+\nu)(9+\nu)\left(-539+3 \nu^{2}\right), \\
& a_{8}^{5, \frac{11}{2}}=-\frac{2}{99225}(-9+\nu)(-7+\nu)(-5+\nu)(5+\nu)(7+\nu)(9+\nu), \quad a_{2}^{6, \frac{3}{2}}=2, \\
& a_{2}^{6, \frac{5}{2}}=2, \quad a_{4}^{6, \frac{5}{2}}=-\frac{2}{81}(-9+\nu)(9+\nu), \quad a_{2}^{6, \frac{7}{2}}=2, a_{4}^{6, \frac{7}{2}}=-\frac{2}{165}\left(-165+\nu^{2}\right), \\
& a_{6}^{6, \frac{7}{2}}=\frac{2}{3969}(-9+\nu)(-7+\nu)(7+\nu)(9+\nu), a_{2}^{6, \frac{9}{2}}=2, a_{4}^{6, \frac{9}{2}}=-\frac{2}{273}\left(-273+\nu^{2}\right), \\
& a_{6}^{6, \frac{9}{2}}=\frac{2}{43659}(-9+\nu)(9+\nu)\left(-539+3 \nu^{2}\right) \text {, } \\
& a_{8}^{6, \frac{9}{2}}=-\frac{2}{99225}(-9+\nu)(-7+\nu)(-5+\nu)(5+\nu)(7+\nu)(9+\nu), \quad a_{2}^{7, \frac{3}{2}}=2, \\
& a_{2}^{7, \frac{5}{2}}=2, \quad a_{4}^{7, \frac{5}{2}}=-\frac{2}{121}(-11+\nu)(11+\nu), \quad a_{2}^{7, \frac{7}{2}}=2, \\
& a_{4}^{7, \frac{7}{2}}=-\frac{2}{715}\left(-715+3 \nu^{2}\right), \quad a_{6}^{7, \frac{7}{2}}=\frac{2}{9801}(-11+\nu)(-9+\nu)(9+\nu)(11+\nu), \\
& a_{2}^{8, \frac{3}{2}}=2, \quad a_{2}^{8, \frac{5}{2}}=2, \quad a_{4}^{8, \frac{5}{2}}=-\frac{2}{169}(-13+\nu)(13+\nu), \quad a_{2}^{9, \frac{3}{2}}=2 .
\end{aligned}
$$

Compared to (3.11), the behavior of this coefficient looks different because there exist some factorized coefficients where each factor has only linear $\nu$ dependent term. For example, for fixed $h_{1}=3$ and $2 r=4$ and different values for $h_{2}$, we observe that there are factors $(-3+$ $\nu)(3+\nu), \cdots,(-13+\nu)(13+\nu)$. For fixed $h_{1}=4$ and $2 r=6$ and different values for $h_{2}$, there are factors $(-5+\nu)(-3+\nu)(3+\nu)(5+\nu), \cdots,(-11+\nu)(-9+\nu)(9+\nu)(11+\nu)$. Furthermore, for fixed $h_{1}=5$ and $2 r=8$, we have two different sextic terms. Then the question is how we can write down the linear combination of two generalized hypergeometric functions leading to the above factorized forms. From the experience of (3.11), we can imagine that the odd terms in $\nu$ of each generalized hypergeometric function should be removed after adding them. That is, the linear, the third order term and the fifth order term in $\nu$ of each generalized hypergeometric function for the above sextic terms are removed. Therefore we should find the appropriate generalized hypergeometric functions which satisfy all these requirements. ${ }^{19}$

Similarly, the structure constants associated with the oscillators (and with the operator

\footnotetext{
${ }^{19}$ We have $\phi_{5}^{3,3}(\mu, 2)=\frac{1}{9}\left(9-2 \nu-\nu^{2}\right)$ corresponding to the coefficient $a_{4}^{3, \frac{5}{2}}$ and its expression by changing $\nu$ into $-\nu$. We can check that $\phi_{5}^{3,3}(\mu, 2)$ looks similar to $\phi_{4}^{3,3}(\mu, 1)$ in the sense that the four upper elements are the same while the last lower element is different from each other. This implies that the $\nu$ dependence is the same (the number of terms in the generalized hypergemetric function is the same) but their overall coefficients of each term are different. Let us emphasize that there are some shifts in the $r$ and $h_{2}$ in (4.2). In this case, we need to substract $\frac{1}{2}$ from $h_{2}=3$ and 1 from $r=5$ in the $\phi_{5}^{3,3}$ in order to obtain the indices of the coefficient $a_{4}^{3, \frac{5}{2}}$. Then we can add these two and obtain the above quadratic term or the coefficient $a_{4}^{3, \frac{5}{2}}$. Moreover we have $\phi_{7}^{4,4}(\mu, 2)=\frac{1}{225}\left(225-76 \nu-34 \nu^{2}+4 \nu^{3}+\nu^{4}\right)$ corresponding to the coefficient $a_{6}^{4, \frac{7}{2}}$ and by similar analysis this will lead to the above quartic term where the odd $\nu$ terms are gone. For the $\phi_{9}^{5,5}(\mu, 2)=\frac{1}{11025}\left(11025-4542 \nu-1891 \nu^{2}+372 \nu^{3}+83 \nu^{4}-6 \nu^{5}-\nu^{6}\right)$ corresponding to the coefficient $a_{8}^{5, \frac{9}{2}}$, we can obtain the sextic term by adding it to $\phi_{9}^{5,5}(1-\mu, 2)$.
} 
$K$ in eq. (C.1)) can be determined as follows:

$$
\begin{aligned}
& b_{3}^{2, \frac{7}{2}}=-\frac{12 \nu}{25}, \quad b_{3}^{2, \frac{9}{2}}=-\frac{12 \nu}{49}, \quad b_{3}^{2, \frac{11}{2}}=-\frac{4 \nu}{27}, \quad b_{3}^{2, \frac{13}{2}}=-\frac{12 \nu}{121}, b_{3}^{2, \frac{15}{2}}=-\frac{12 \nu}{169}, \\
& b_{3}^{2, \frac{17}{2}}=-\frac{4 \nu}{75}, \quad b_{3}^{3, \frac{5}{2}}=-\frac{4 \nu}{15}, \quad b_{3}^{3, \frac{7}{2}}=-\frac{4 \nu}{35}, \quad b_{3}^{3, \frac{9}{2}}=-\frac{4 \nu}{63}, \\
& b_{5}^{3, \frac{9}{2}}=\frac{8}{735} \nu\left(-47+3 \nu^{2}\right), \quad b_{3}^{3, \frac{11}{2}}=-\frac{4 \nu}{99}, \quad b_{5}^{3, \frac{11}{2}}=\frac{40}{3969}(-5+\nu) \nu(5+\nu), \\
& b_{3}^{3, \frac{13}{2}}=-\frac{4 \nu}{143}, \quad b_{5}^{3, \frac{13}{2}}=\frac{40}{9801} \nu\left(-37+\nu^{2}\right), \quad b_{3}^{3, \frac{15}{2}}=-\frac{4 \nu}{195}, \\
& b_{5}^{3, \frac{15}{2}}=\frac{40}{61347} \nu\left(-155+3 \nu^{2}\right), \quad b_{3}^{4, \frac{5}{2}}=-\frac{4 \nu}{35}, \quad b_{3}^{4, \frac{7}{2}}=-\frac{4 \nu}{75}, \\
& b_{5}^{4, \frac{7}{2}}=\frac{8}{525}(-5+\nu) \nu(5+\nu), \quad b_{3}^{4, \frac{9}{2}}=-\frac{12 \nu}{385}, \quad b_{5}^{4, \frac{9}{2}}=\frac{8}{2205}(-7+\nu) \nu(7+\nu), \\
& b_{3}^{4, \frac{11}{2}}=-\frac{4 \nu}{195}, \quad b_{5}^{4, \frac{11}{2}}=\frac{8}{6237}(-9+\nu) \nu(9+\nu), b_{7}^{4, \frac{11}{2}}=-\frac{4}{4725}(-5+\nu)^{2} \nu(5+\nu)^{2}, \\
& b_{3}^{4, \frac{13}{2}}=-\frac{4 \nu}{275}, \quad b_{5}^{4, \frac{13}{2}}=\frac{8}{14157}(-11+\nu) \nu(11+\nu), \\
& b_{7}^{4, \frac{13}{2}}=-\frac{4}{343035} \nu\left(22751-1070 \nu^{2}+15 \nu^{4}\right), \quad b_{3}^{5, \frac{5}{2}}=-\frac{4 \nu}{63}, \quad b_{3}^{5, \frac{7}{2}}=-\frac{12 \nu}{385}, \\
& \left.b_{5}^{5, \frac{7}{2}}=\frac{8}{2205}(-7+\nu) \nu(7+\nu)\right), \quad b_{3}^{5, \frac{9}{2}}=-\frac{12 \nu}{637}, \quad b_{5}^{5, \frac{9}{2}}=\frac{8}{24255} \nu\left(-287+3 \nu^{2}\right), \\
& b_{7}^{5, \frac{9}{2}}=-\frac{4}{11025}(-7+\nu)(-5+\nu) \nu(5+\nu)(7+\nu), \quad b_{3}^{5, \frac{11}{2}}=-\frac{4 \nu}{315}, \\
& b_{5}^{5, \frac{11}{2}}=\frac{8}{63063} \nu\left(-467+3 \nu^{2}\right), \quad b_{7}^{5, \frac{11}{2}}=-\frac{4}{72765}(-9+\nu)(-7+\nu) \nu(7+\nu)(9+\nu), \\
& b_{3}^{6, \frac{5}{2}}=-\frac{4 \nu}{99}, \quad b_{3}^{6, \frac{7}{2}}=-\frac{4 \nu}{195}, \quad b_{5}^{6, \frac{7}{2}}=\frac{8}{6237}(-9+\nu) \nu(9+\nu), \quad b_{3}^{6, \frac{9}{2}}=-\frac{4 \nu}{315}, \\
& b_{5}^{6, \frac{9}{2}}=\frac{8}{63063} \nu\left(-467+3 \nu^{2}\right), \quad b_{7}^{6, \frac{9}{2}}=-\frac{4}{72765}(-9+\nu)(-7+\nu) \nu(7+\nu)(9+\nu), \\
& b_{3}^{7, \frac{5}{2}}=-\frac{4 \nu}{143}, \quad b_{3}^{7, \frac{7}{2}}=-\frac{4 \nu}{275}, \quad b_{5}^{7, \frac{7}{2}}=\frac{8}{14157}(-11+\nu) \nu(11+\nu), \\
& b_{3}^{8, \frac{5}{2}}=-\frac{4 \nu}{195} \text {. }
\end{aligned}
$$

In particular, we observe that some of the coefficients in eq. (C.3) are equal to the ones in (3.12). Let us focus on the cubic term in $\nu$. For example, the coefficient $b_{5}^{4, \frac{7}{2}}$ in eq. (C.3) is the same as $b_{6}^{4,4}$ in (3.12). Then we can try to determine the explicit form for the generalized hypergeometric function by following the procedure around (3.12). The corresponding quartic terms $(\nu \mp 3)(\nu \pm 5)(\nu \mp 5)(\nu \pm 7)$ for the above cubic term can arise in the generalized hypergeometric functions. Moreover, the sextic terms $(\nu \pm 5)(\nu \mp 7)(\nu \pm 7)(\nu \mp$ $9)(\nu \pm 9)(\nu \mp 11)$ for the above quintic term in eq. (C.3) can arise in the generalized hypergeometric functions. ${ }^{20}$

\footnotetext{
${ }^{20}$ We have $\phi_{4}^{3,3}(\mu, 1)=-\frac{1}{15}(-3+\nu)(5+\nu), \phi_{6}^{4,4}(\mu, 1)=\frac{1}{525}(-5+\nu)(-3+\nu)(5+\nu)(7+\nu)$, and $\phi_{8}^{5,5}(\mu, 1)=$ $-\frac{1}{33075}(-7+\nu)(-5+\nu)(-3+\nu)(5+\nu)(7+\nu)(9+\nu)$ from the analysis of (3.11). In this case, by subtracting the corresponding generalized hypergeometric functions replaced by $\nu \rightarrow-\nu$ respectively from the above, we obtain the coefficients $b_{3}^{3, \frac{5}{2}}, b_{5}^{4, \frac{7}{2}}$ and $b_{7}^{5, \frac{9}{2}}$.
} 
Then we can rewrite these structure constants in terms of generalized hypergeometric functions. It turns out, from eqs. (C.2) and (C.3), that

$$
\begin{aligned}
a_{2 r}^{h_{1}, h_{2}+\frac{1}{2}}(\mu)= & { }_{4} F_{3}\left[\begin{array}{c|c}
\frac{1}{2}+\mu, \frac{1}{2}-\mu, \frac{2-2 r}{2}, \frac{1-2 r}{2} \\
\frac{3}{2}-h_{1}, \frac{1}{2}-h_{2}, \frac{1}{2}+h_{1}+h_{2}-2 r \mid
\end{array}\right] \\
& +{ }_{4} F_{3}\left[\begin{array}{c|c}
\frac{3}{2}-\mu,-\frac{1}{2}+\mu, \frac{2-2 r}{2}, \frac{1-2 r}{2} \\
\frac{3}{2}-h_{1}, \frac{1}{2}-h_{2}, \frac{1}{2}+h_{1}+h_{2}-2 r \mid
\end{array}\right], \\
b_{2 r-1}^{h_{1}, h_{2}+\frac{1}{2}}(\mu)= & { }_{4} F_{3}\left[\begin{array}{c}
\frac{1}{2}+\mu, \frac{1}{2}-\mu, \frac{2-2 r}{2}, \frac{1-2 r}{2} \\
\frac{3}{2}-h_{1}, \frac{1}{2}-h_{2}, \frac{3}{2}+h_{1}+h_{2}-2 r \mid
\end{array}\right] \\
& -{ }_{4} F_{3}\left[\begin{array}{c}
\frac{3}{2}-\mu,-\frac{1}{2}+\mu, \frac{2-2 r}{2}, \frac{1-2 r}{2} \\
\frac{3}{2}-h_{1}, \frac{1}{2}-h_{2}, \frac{3}{2}+h_{1}+h_{2}-2 r \mid
\end{array}\right] .
\end{aligned}
$$

The structure constant $b_{2 r-1}^{h_{1}, h_{2}+\frac{1}{2}}(\mu)$ contains the generalized hypergeometric function of "saalschutzian" in the sense that the sum of upper elements plus 1 is equal to the sum of lower elements. On the other hand, the structure constant $a_{2 r}^{h_{1}, h_{2}+\frac{1}{2}}(\mu)$ is not "saalschutzian". In order to compare with (3.13), let us look at the first relation of eq. (C.4). By replacing $h_{2} \rightarrow h_{2}-1$ and $2 r \rightarrow 2 r-1$ in the generalized hypergeometric functions, we observe that this becomes the first relation of (3.13) where the third and fourth upper elements of generalized hypergeometric functions are shifted by $\frac{1}{2}$. For the second relation of eq. (C.4), by replacing $h_{2} \rightarrow h_{2}-1$, this becomes the second relation of (3.13). We will see that the above two structure constants in eq. (C.4) are denoted by the second one in $(4.2)$.

\section{C.1.2 Operator $K$ dependence in the oscillators}

We can analyze the other case corresponding to (3.6) as follows. By writing the $\nu$ dependent structure constants, we obtain

$$
\begin{aligned}
& {[\underbrace{\hat{y}_{(1} \ldots \hat{y}_{1}}_{h_{1}-1+m} \underbrace{\left.\hat{y}_{2} \ldots \hat{y}_{2}\right)}_{h_{1}-1-m} K, \underbrace{\hat{y}_{(1} \ldots \hat{y}_{1}}_{h_{2}-\frac{1}{2}+\rho} \underbrace{\hat{y}_{2} \ldots \hat{y}_{2)}}_{h_{2}-\frac{1}{2}-\rho}]=} \\
& \sum_{r=1}^{\left[\frac{h_{1}+h_{2}}{2}\right]}(-1)^{r}[N_{2 r-1}^{h_{1} h_{2}+\frac{1}{2}}(m, \rho) \frac{1}{(2 r-2) !} a_{2 r-1}^{h_{1}, h_{2}+\frac{1}{2}}(\nu) \underbrace{\hat{y}_{(1} \ldots \ldots \ldots \ldots \hat{y}_{1}}_{h_{1}+h_{2}-2 r+\frac{1}{2}+m+\rho} \underbrace{\left.\hat{y}_{2} \ldots \ldots \ldots \ldots \hat{y}_{2}\right)}_{h_{1}+h_{2}-2 r+\frac{1}{2}-m-\rho} K] \\
& -\sum_{r=1}^{\left[\frac{h_{1}+h_{2}-1}{2}\right]}(-1)^{r}[\mathrm{i} N_{2 r}^{h_{1} h_{2}+\frac{1}{2}}(m, \rho) \frac{\left(h_{1}-r-\frac{1}{2}\right)}{r(2 r-1) !} b_{2 r}^{h_{1}, h_{2}+\frac{1}{2}}(\nu) \underbrace{\hat{y}_{(1} \ldots \ldots \ldots \ldots \hat{y}_{1}}_{h_{1}+h_{2}-2 r-\frac{1}{2}+m+\rho} \underbrace{\hat{y}_{2} \ldots \ldots \ldots \ldots \hat{y}_{2)}}_{h_{1}+h_{2}-2 r-\frac{1}{2}-m-\rho}] \text {. }
\end{aligned}
$$

As done before, the results for the coefficient $a_{2 r-1}^{h_{1}, h_{2}+\frac{1}{2}}(\nu)$ are, from eq. (C.5), given by

$$
\begin{aligned}
& a_{1}^{1, \frac{5}{2}}=2, \quad a_{1}^{1, \frac{7}{2}}=2, \quad a_{1}^{1, \frac{9}{2}}=2, \quad a_{1}^{1, \frac{11}{2}}=2, \quad a_{1}^{1, \frac{13}{2}}=2, \quad a_{1}^{1, \frac{15}{2}}=2, \\
& a_{1}^{1, \frac{17}{2}}=2, \quad a_{1}^{1, \frac{19}{2}}=2, \quad a_{1}^{2, \frac{3}{2}}=2, \quad a_{1}^{2, \frac{5}{2}}=2, \quad a_{1}^{2, \frac{7}{2}}=2,
\end{aligned}
$$




$$
\begin{aligned}
& a_{3}^{2, \frac{7}{2}}=-\frac{2}{25}(-5+\nu)(5+\nu), \quad a_{1}^{2, \frac{9}{2}}=2, \quad a_{3}^{2, \frac{9}{2}}=-\frac{2}{49}(-7+\nu)(7+\nu), \\
& a_{1}^{2, \frac{11}{2}}=2, \quad a_{3}^{2, \frac{11}{2}}=-\frac{2}{81}(-9+\nu)(9+\nu), \quad a_{1}^{2, \frac{13}{2}}=2, \\
& a_{3}^{2, \frac{13}{2}}=-\frac{2}{121}(-11+\nu)(11+\nu), \quad a_{1}^{2, \frac{15}{2}}=2, a_{3}^{2, \frac{15}{2}}=-\frac{2}{169}(-13+\nu)(13+\nu), \\
& a_{1}^{2, \frac{17}{2}}=2, \quad a_{3}^{2, \frac{17}{2}}=-\frac{2}{225}(-15+\nu)(15+\nu), \quad a_{1}^{3, \frac{3}{2}}=2, \quad a_{1}^{3, \frac{5}{2}}=2, \\
& a_{3}^{3, \frac{5}{2}}=-\frac{2}{45}\left(-45+\nu^{2}\right), \quad a_{1}^{3, \frac{7}{2}}=2, \quad a_{3}^{3, \frac{7}{2}}=-\frac{2}{105}\left(-105+\nu^{2}\right), \quad a_{1}^{3, \frac{9}{2}}=2, \\
& a_{3}^{3, \frac{9}{2}}=-\frac{2}{189}\left(-189+\nu^{2}\right), \quad a_{5}^{3, \frac{9}{2}}=\frac{2}{1225}(-7+\nu)(-5+\nu)(5+\nu)(7+\nu), \\
& a_{1}^{3, \frac{11}{2}}=2, \quad a_{3}^{3, \frac{11}{2}}=-\frac{2}{297}\left(-297+\nu^{2}\right), \\
& a_{5}^{3, \frac{11}{2}}=\frac{2}{3969}(-9+\nu)(-7+\nu)(7+\nu)(9+\nu), \quad a_{1}^{3, \frac{13}{2}}=2, \\
& a_{3}^{3, \frac{13}{2}}=-\frac{2}{429}\left(-429+\nu^{2}\right), \quad a_{5}^{3, \frac{13}{2}}=\frac{2}{9801}(-11+\nu)(-9+\nu)(9+\nu)(11+\nu), \\
& a_{1}^{3, \frac{15}{2}}=2, \quad a_{3}^{3, \frac{15}{2}}=-\frac{2}{585}\left(-585+\nu^{2}\right), \\
& a_{5}^{3, \frac{15}{2}}=\frac{2}{20449}(-13+\nu)(-11+\nu)(11+\nu)(13+\nu), \quad a_{1}^{4, \frac{3}{2}}=2, \quad a_{1}^{4, \frac{5}{2}}=2, \\
& a_{3}^{4, \frac{5}{2}}=-\frac{2}{105}\left(-105+\nu^{2}\right), \quad a_{1}^{4, \frac{7}{2}}=2, \quad a_{3}^{4, \frac{7}{2}}=-\frac{2}{225}(-15+\nu)(15+\nu), \\
& a_{5}^{4, \frac{7}{2}}=\frac{2}{2625}(-5+\nu)(5+\nu)\left(-105+\nu^{2}\right), \quad a_{1}^{4, \frac{9}{2}}=2, a_{3}^{4, \frac{9}{2}}=-\frac{2}{385}\left(-385+\nu^{2}\right), \\
& a_{5}^{4, \frac{9}{2}}=\frac{2}{11025}(-15+\nu)(-7+\nu)(7+\nu)(15+\nu), \quad a_{1}^{4, \frac{11}{2}}=2, \\
& a_{3}^{4, \frac{11}{2}}=-\frac{2}{585}\left(-585+\nu^{2}\right), \quad a_{5}^{4, \frac{11}{2}}=\frac{2}{31185}(-9+\nu)(9+\nu)\left(-385+\nu^{2}\right), \\
& a_{7}^{4, \frac{11}{2}}=-\frac{2}{99225}(-9+\nu)(-7+\nu)(-5+\nu)(5+\nu)(7+\nu)(9+\nu), \quad a_{1}^{4, \frac{13}{2}}=2, \\
& a_{3}^{4, \frac{13}{2}}=-\frac{2}{825}\left(-825+\nu^{2}\right), \quad a_{5}^{4, \frac{13}{2}}=\frac{2}{70785}(-11+\nu)(11+\nu)\left(-585+\nu^{2}\right), \\
& a_{7}^{4, \frac{13}{2}}=-\frac{2}{480249}(-11+\nu)(-9+\nu)(-7+\nu)(7+\nu)(9+\nu)(11+\nu), \quad a_{1}^{5, \frac{3}{2}}=2, \\
& a_{1}^{5, \frac{5}{2}}=2, \quad a_{3}^{5, \frac{5}{2}}=-\frac{2}{189}\left(-189+\nu^{2}\right), \quad a_{1}^{5, \frac{7}{2}}=2, a_{3}^{5, \frac{7}{2}}=-\frac{2}{385}\left(-385+\nu^{2}\right), \\
& a_{5}^{5, \frac{7}{2}}=\frac{2}{11025}(-15+\nu)(-7+\nu)(7+\nu)(15+\nu), \quad a_{1}^{5, \frac{9}{2}}=2, \\
& a_{3}^{5, \frac{9}{2}}=-\frac{2}{637}\left(-637+\nu^{2}\right), \quad a_{5}^{5, \frac{9}{2}}=\frac{2}{40425}\left(40425-554 \nu^{2}+\nu^{4}\right), \\
& a_{7}^{5, \frac{9}{2}}=-\frac{2}{231525}(-7+\nu)(-5+\nu)(5+\nu)(7+\nu)\left(-189+\nu^{2}\right), \quad a_{1}^{5, \frac{11}{2}}=2, \\
& a_{3}^{5, \frac{11}{2}}=-\frac{2}{945}\left(-945+\nu^{2}\right), \quad a_{5}^{5, \frac{11}{2}}=\frac{2}{105105}\left(105105-914 \nu^{2}+\nu^{4}\right), \\
& a_{7}^{5, \frac{11}{2}}=-\frac{2}{1528065}(-9+\nu)(-7+\nu)(7+\nu)(9+\nu)\left(-385+\nu^{2}\right), \quad a_{1}^{6, \frac{3}{2}}=2, \\
& a_{1}^{6, \frac{5}{2}}=2, \quad a_{3}^{6, \frac{5}{2}}=-\frac{2}{297}\left(-297+\nu^{2}\right), \quad a_{1}^{6, \frac{7}{2}}=2, \quad a_{3}^{6, \frac{7}{2}}=-\frac{2}{585}\left(-585+\nu^{2}\right),
\end{aligned}
$$




$$
\begin{aligned}
a_{5}^{6, \frac{7}{2}} & =\frac{2}{31185}(-9+\nu)(9+\nu)\left(-385+\nu^{2}\right), \quad a_{1}^{6, \frac{9}{2}}=2, a_{3}^{6, \frac{9}{2}}=-\frac{2}{945}\left(-945+\nu^{2}\right), \\
a_{5}^{6, \frac{9}{2}} & =\frac{2}{105105}\left(105105-914 \nu^{2}+\nu^{4}\right), \\
a_{7}^{6, \frac{9}{2}} & =-\frac{2}{1528065}(-9+\nu)(-7+\nu)(7+\nu)(9+\nu)\left(-385+\nu^{2}\right), a_{1}^{7, \frac{3}{2}}=2, a_{1}^{7, \frac{5}{2}}=2 \\
a_{3}^{7, \frac{5}{2}} & =-\frac{2}{429}\left(-429+\nu^{2}\right), \quad a_{1}^{7, \frac{7}{2}}=2, \quad a_{3}^{7, \frac{7}{2}}=-\frac{2}{825}\left(-825+\nu^{2}\right) \\
a_{5}^{7, \frac{7}{2}} & =\frac{2}{70785}(-11+\nu)(11+\nu)\left(-585+\nu^{2}\right), \quad a_{1}^{8, \frac{3}{2}}=2, \quad a_{1}^{8, \frac{5}{2}}=2 \\
a_{3}^{8, \frac{5}{2}} & =-\frac{2}{585}\left(-585+\nu^{2}\right), \quad a_{1}^{9, \frac{3}{2}}=2 .
\end{aligned}
$$

We can easily see that some of the coefficients in eq. (C.6) overlap with the ones in eq. (C.2). For example, the coefficient $a_{3}^{2, \frac{7}{2}}$ of eq. (C.6) is exactly the same as the coefficient $a_{3}^{3, \frac{7}{2}}$ of eq. (C.2). We can do similar analysis and will arrive at the explicit $\nu$ dependence in the generalized hypergeometric functions we are considering.

Similarly, the coefficient $b_{2 r}^{h_{1}, h_{2}+\frac{1}{2}}(\nu)$ can be summarized by

$$
\begin{aligned}
& b_{2}^{2, \frac{3}{2}}=-\frac{4 \nu}{3}, \quad b_{2}^{2, \frac{5}{2}}=-\frac{4 \nu}{15}, \quad b_{2}^{2, \frac{7}{2}}=-\frac{4 \nu}{35}, \quad b_{2}^{2, \frac{9}{2}}=-\frac{4 \nu}{63}, \quad b_{2}^{2, \frac{11}{2}}=-\frac{4 \nu}{99}, \\
& b_{2}^{2, \frac{13}{2}}=-\frac{4 \nu}{143}, \quad b_{2}^{2, \frac{15}{2}}=-\frac{4 \nu}{195}, \quad b_{2}^{2, \frac{17}{2}}=-\frac{4 \nu}{255}, \quad b_{2}^{3, \frac{3}{2}}=-\frac{4 \nu}{15}, b_{2}^{3, \frac{5}{2}}=-\frac{4 \nu}{63}, \\
& b_{4}^{3, \frac{5}{2}}=\frac{8}{45}(-3+\nu) \nu(3+\nu), \quad b_{2}^{3, \frac{7}{2}}=-\frac{4 \nu}{135}, \quad b_{4}^{3, \frac{7}{2}}=\frac{8}{525}(-5+\nu) \nu(5+\nu), \\
& b_{2}^{3, \frac{9}{2}}=-\frac{4 \nu}{231}, \quad b_{4}^{3, \frac{9}{2}}=\frac{8}{2205}(-7+\nu) \nu(7+\nu), \quad b_{2}^{3, \frac{11}{2}}=-\frac{4 \nu}{351}, \\
& b_{4}^{3, \frac{11}{2}}=\frac{8}{6237}(-9+\nu) \nu(9+\nu), \quad b_{2}^{3, \frac{13}{2}}=-\frac{4 \nu}{495}, b_{4}^{3, \frac{13}{2}}=\frac{8}{14157}(-11+\nu) \nu(11+\nu), \\
& b_{2}^{3, \frac{15}{2}}=-\frac{4 \nu}{663}, \quad b_{4}^{3, \frac{15}{2}}=\frac{8}{27885}(-13+\nu) \nu(13+\nu), \quad b_{2}^{4, \frac{3}{2}}=-\frac{4 \nu}{35}, \\
& b_{2}^{4, \frac{5}{2}}=-\frac{4 \nu}{135}, \quad b_{4}^{4, \frac{5}{2}}=\frac{8}{525}(-5+\nu) \nu(5+\nu), \quad b_{2}^{4, \frac{7}{2}}=-\frac{4 \nu}{275}, \\
& b_{4}^{4, \frac{7}{2}}=\frac{8}{4725} \nu\left(-85+\nu^{2}\right), \quad b_{6}^{4, \frac{7}{2}}=-\frac{4}{525}(-5+\nu)(-3+\nu) \nu(3+\nu)(5+\nu), \\
& b_{2}^{4, \frac{9}{2}}=-\frac{4 \nu}{455}, \quad b_{4}^{4, \frac{9}{2}}=\frac{8}{17325}(-13+\nu) \nu(13+\nu), \\
& b_{6}^{4, \frac{9}{2}}=-\frac{4}{11025}(-7+\nu)(-5+\nu) \nu(5+\nu)(7+\nu), \quad b_{2}^{4, \frac{11}{2}}=-\frac{4 \nu}{675}, \\
& b_{4}^{4, \frac{11}{2}}=\frac{8}{45045} \nu\left(-277+\nu^{2}\right), \quad b_{6}^{4, \frac{11}{2}}=-\frac{4}{72765}(-9+\nu)(-7+\nu) \nu(7+\nu)(9+\nu), \\
& b_{2}^{4, \frac{13}{2}}=-\frac{4 \nu}{935}, \quad b_{4}^{4, \frac{13}{2}}=\frac{8}{96525} \nu\left(-409+\nu^{2}\right), \\
& b_{6}^{4, \frac{13}{2}}=-\frac{4}{297297}(-11+\nu)(-9+\nu) \nu(9+\nu)(11+\nu), b_{2}^{5, \frac{3}{2}}=-\frac{4 \nu}{63}, \quad b_{2}^{5, \frac{5}{2}}=-\frac{4 \nu}{231}, \\
& b_{4}^{5, \frac{5}{2}}=\frac{8}{2205}(-7+\nu) \nu(7+\nu), \quad b_{2}^{5, \frac{7}{2}}=-\frac{4 \nu}{455}, b_{4}^{5, \frac{7}{2}}=\frac{8}{17325}(-13+\nu) \nu(13+\nu), \\
& b_{6}^{5, \frac{7}{2}}=-\frac{4}{11025}(-7+\nu)(-5+\nu) \nu(5+\nu)(7+\nu), \quad b_{2}^{5, \frac{9}{2}}=-\frac{4 \nu}{735},
\end{aligned}
$$




$$
\begin{aligned}
b_{4}^{5, \frac{9}{2}} & =\frac{24}{175175} \nu\left(-329+\nu^{2}\right), b_{6}^{5, \frac{9}{2}}=-\frac{4}{169785}(-13+\nu)(-7+\nu) \nu(7+\nu)(13+\nu), \\
b_{8}^{5, \frac{9}{2}} & =\frac{16}{99225}(-7+\nu)(-5+\nu)(-3+\nu) \nu(3+\nu)(5+\nu)(7+\nu), b_{2}^{5, \frac{11}{2}}=-\frac{4 \nu}{1071}, \\
b_{4}^{5, \frac{11}{2}} & =\frac{8}{143325}(-23+\nu) \nu(23+\nu), b_{6}^{5, \frac{11}{2}}=-\frac{4}{945945}(-9+\nu) \nu(9+\nu)\left(-329+\nu^{2}\right), \\
b_{8}^{5, \frac{11}{2}} & =\frac{16}{3274425}(-9+\nu)(-7+\nu)(-5+\nu) \nu(5+\nu)(7+\nu)(9+\nu), \quad b_{2}^{6, \frac{3}{2}}=-\frac{4 \nu}{99}, \\
b_{2}^{6, \frac{5}{2}} & =-\frac{4 \nu}{351}, \quad b_{4}^{6, \frac{5}{2}}=\frac{8}{6237}(-9+\nu) \nu(9+\nu), \quad b_{2}^{6, \frac{7}{2}}=-\frac{4 \nu}{675}, \\
b_{4}^{6, \frac{7}{2}} & =\frac{8}{45045} \nu\left(-277+\nu^{2}\right), \quad b_{6}^{6, \frac{7}{2}}=-\frac{4}{72765}(-9+\nu)(-7+\nu) \nu(7+\nu)(9+\nu), \\
b_{2}^{6, \frac{9}{2}} & =-\frac{4 \nu}{1071}, \quad b_{4}^{6, \frac{9}{2}}=\frac{8}{143325}(-23+\nu) \nu(23+\nu), \\
b_{6}^{6, \frac{9}{2}} & =-\frac{4}{945945}(-9+\nu) \nu(9+\nu)\left(-329+\nu^{2}\right), \quad b_{2}^{7, \frac{3}{2}}=-\frac{4 \nu}{143}, \\
b_{8}^{6, \frac{9}{2}} & =\frac{16}{3274425}(-9+\nu)(-7+\nu)(-5+\nu) \nu(5+\nu)(7+\nu)(9+\nu), \quad b_{2}^{7, \frac{7}{2}}=-\frac{4 \nu}{935}, \\
b_{2}^{7, \frac{5}{2}} & =-\frac{4 \nu}{495}, \quad b_{4}^{7, \frac{5}{2}}=\frac{8}{14157}(-11+\nu) \nu(11+\nu), \\
b_{4}^{7, \frac{7}{2}} & =\frac{8}{96525} \nu\left(-409+\nu^{2}\right), \quad b_{6}^{7, \frac{7}{2}}=-\frac{4}{297297}(-11+\nu)(-9+\nu) \nu(9+\nu)(11+\nu), \\
b_{2}^{8, \frac{3}{2}} & =-\frac{4 \nu}{195}, \quad b_{2}^{8, \frac{5}{2}}=-\frac{4 \nu}{663}, \quad b_{4}^{8, \frac{5}{2}}=\frac{8}{27885}(-13+\nu) \nu(13+\nu), \\
b_{2}^{9, \frac{3}{2}} & =-\frac{4 \nu}{255} . \quad
\end{aligned}
$$

Some of the coefficients in eq. (C.7) overlap with the ones in (3.12). For example, the coefficient $b_{4}^{3, \frac{7}{2}}$ of eq. (C.7) is exactly the same as the coefficient $b_{6}^{4,4}$ of (3.12). We can do similar analysis and the explicit $\nu$ dependence in the generalized hypergeometric functions can be determined.

From the results of eqs. (C.6) and (C.7), the general expressions are given by

$$
\begin{aligned}
a_{2 r-1}^{h_{1}, h_{2}+\frac{1}{2}}(\mu)= & { }_{4} F_{3}\left[\begin{array}{c|c}
\frac{1}{2}+\mu, \frac{1}{2}-\mu, \frac{3-2 r}{2}, \frac{2-2 r}{2} \\
\frac{3}{2}-h_{1}, \frac{1}{2}-h_{2}, \frac{3}{2}+h_{1}+h_{2}-2 r \mid 1
\end{array}\right] \\
& +{ }_{4} F_{3}\left[\begin{array}{c|c}
\frac{3}{2}-\mu,-\frac{1}{2}+\mu, \frac{3-2 r}{2}, \frac{2-2 r}{2} \\
\frac{3}{2}-h_{1}, \frac{1}{2}-h_{2}, \frac{3}{2}+h_{1}+h_{2}-2 r \mid
\end{array}\right], \\
b_{2 r}^{h_{1}, h_{2}+\frac{1}{2}}(\mu)= & { }_{4} F_{3}\left[\begin{array}{c}
\frac{1}{2}+\mu, \frac{1}{2}-\mu, \frac{1-2 r}{2}, \frac{-2 r}{2} \\
\frac{3}{2}-h_{1}, \frac{1}{2}-h_{2}, \frac{1}{2}+h_{1}+h_{2}-2 r
\end{array} \mid 1\right] \\
& -{ }_{4} F_{3}\left[\begin{array}{c}
\frac{3}{2}-\mu,-\frac{1}{2}+\mu, \frac{1-2 r}{2}, \frac{-2 r}{2} \\
\frac{3}{2}-h_{1}, \frac{1}{2}-h_{2}, \frac{1}{2}+h_{1}+h_{2}-2 r \mid
\end{array}\right] .
\end{aligned}
$$

We can check that by replacing $2 r$ by $(2 r+1)$ for the coefficient $a$ and $2 r$ by $(2 r-1)$ for the coefficient $b$, the above expression eq. (C.8) becomes the ones in eq. (C.4) and we can see this result in (4.6) and (4.7). 


\section{C.2 The case-three with two fermionic oscillators}

\section{C.2.1 No operator $K$ dependence in the oscillators}

From (3.7), we introduce $\mu$ dependent structure constants as follows:

$$
\begin{aligned}
& \{\underbrace{\hat{y}_{(1} \ldots \hat{y}_{1}}_{h_{1}-\frac{1}{2}+\rho} \underbrace{\hat{y}_{2} \ldots \hat{y}_{2)}}_{h_{1}-\frac{1}{2}-\rho}, \underbrace{\hat{y}_{(1} \ldots \hat{y}_{1}}_{h_{2}-\frac{1}{2}+\omega} \underbrace{\left.\hat{y}_{2} \ldots \hat{y}_{2}\right)}_{h_{2}-\frac{1}{2}-\omega}\}= \\
& {\left[\frac{\left.\frac{h_{1}+h_{2}+1}{2}\right]}{\sum_{r=1}^{2}}(-1)^{r} N_{2 r-1}^{h_{1}+\frac{1}{2} h_{2}+\frac{1}{2}}(\rho, \omega) \frac{(-1)}{(2 r-2) !}[a_{2 r-1}^{h_{1}+\frac{1}{2}, h_{2}+\frac{1}{2}}(\nu) \underbrace{\hat{y}_{(1} \ldots \ldots \ldots \hat{y}_{1}}_{h_{1}+h_{2}-2 r+1+\rho+\omega} \underbrace{\left.\hat{y}_{2} \ldots \ldots \ldots \hat{y}_{2}\right)}_{h_{1}+h_{2}-2 r+1-\rho-\omega}\right.} \\
& -\frac{2\left(h_{1}+h_{2}-r+1\right)}{(2 r-1)} b_{2 r-1}^{h_{1}+\frac{1}{2}, h_{2}+\frac{1}{2}}(\nu) \underbrace{\hat{y}_{(1} \ldots \ldots \ldots \hat{y}_{1}}_{h_{1}+h_{2}-2 r+1+\rho+\omega} \underbrace{\left.\hat{y}_{2} \ldots \ldots \ldots \hat{y}_{2}\right)}_{h_{1}+h_{2}-2 r+1-\rho-\omega} K] \text {. }
\end{aligned}
$$

We can compute the anticommutator for several values of $\left(h_{1}, h_{2}\right)$. We can determine the structure constants having the operator $K$ independent oscillators in eq. (C.9) as follows:

$$
\begin{aligned}
& a_{1}^{\frac{3}{2}, \frac{3}{2}}=2, \quad a_{1}^{\frac{3}{2}, \frac{5}{2}}=2, \quad a_{1}^{\frac{3}{2}, \frac{7}{2}}=2, \quad a_{1}^{\frac{3}{2}, \frac{9}{2}}=2, \quad a_{1}^{\frac{3}{2}, \frac{11}{2}}=2, \quad a_{1}^{\frac{3}{2}, \frac{13}{2}}=2, \\
& a_{1}^{\frac{3}{2}, \frac{15}{2}}=2, \quad a_{1}^{\frac{3}{2}, \frac{17}{2}}=2, \quad a_{1}^{\frac{3}{2}, \frac{19}{2}}=2, \quad a_{1}^{\frac{5}{2}, \frac{3}{2}}=2, \\
& a_{1}^{\frac{5}{2}, \frac{5}{2}}=2, \quad a_{3}^{\frac{5}{2}, \frac{5}{2}}=-\frac{2}{45}\left(-45+\nu^{2}\right), \quad a_{1}^{\frac{5}{2}, \frac{7}{2}}=2, \quad a_{3}^{\frac{5}{2}, \frac{7}{2}}=-\frac{2}{105}\left(-105+\nu^{2}\right), \\
& a_{1}^{\frac{5}{2}, \frac{9}{2}}=2, \quad a_{3}^{\frac{5}{2}, \frac{9}{2}}=-\frac{2}{189}\left(-189+\nu^{2}\right), \quad a_{1}^{\frac{5}{2}, \frac{11}{2}}=2, a_{3}^{\frac{5}{2}, \frac{11}{2}}=-\frac{2}{297}\left(-297+\nu^{2}\right), \\
& a_{1}^{\frac{5}{2}, \frac{13}{2}}=2, \quad a_{3}^{\frac{5}{2}, \frac{13}{2}}=-\frac{2}{429}\left(-429+\nu^{2}\right), \quad a_{1}^{\frac{5}{2}, \frac{15}{2}}=2, a_{3}^{\frac{5}{2}, \frac{15}{2}}=-\frac{2}{585}\left(-585+\nu^{2}\right), \\
& a_{1}^{\frac{5}{2}, \frac{17}{2}}=2, \quad a_{3}^{\frac{5}{2}, \frac{17}{2}}=-\frac{2}{765}\left(-765+\nu^{2}\right), \quad a_{1}^{\frac{7}{2}, \frac{3}{2}}=2, \quad a_{1}^{\frac{7}{2}, \frac{5}{2}}=2, \\
& a_{3}^{\frac{7}{2}, \frac{5}{2}}=-\frac{2}{105}\left(-105+\nu^{2}\right), \quad a_{1}^{\frac{7}{2}, \frac{7}{2}}=2, \quad a_{3}^{\frac{7}{2}, \frac{7}{2}}=-\frac{2}{225}(-15+\nu)(15+\nu), \\
& a_{5}^{\frac{7}{2}, \frac{7}{2}}=\frac{2}{2625}(-5+\nu)(5+\nu)\left(-105+\nu^{2}\right), \quad a_{1}^{\frac{7}{2}, \frac{9}{2}}=2, a_{3}^{\frac{7}{2}, \frac{9}{2}}=-\frac{2}{385}\left(-385+\nu^{2}\right), \\
& a_{5}^{\frac{7}{2}, \frac{9}{2}}=\frac{2}{11025}(-15+\nu)(-7+\nu)(7+\nu)(15+\nu), \quad a_{1}^{\frac{7}{2}, \frac{11}{2}}=2, \\
& a_{3}^{\frac{7}{2}, \frac{11}{2}}=-\frac{2}{585}\left(-585+\nu^{2}\right), \quad a_{5}^{\frac{7}{2}, \frac{11}{2}}=\frac{2}{31185}(-9+\nu)(9+\nu)\left(-385+\nu^{2}\right), \\
& a_{1}^{\frac{7}{2}, \frac{13}{2}}=2, \quad a_{3}^{\frac{7}{2}, \frac{13}{2}}=-\frac{2}{825}\left(-825+\nu^{2}\right) \\
& a_{5}^{\frac{7}{2}, \frac{13}{2}}=\frac{2}{70785}(-11+\nu)(11+\nu)\left(-585+\nu^{2}\right), \quad a_{1}^{\frac{7}{2}, \frac{15}{2}}=2, \\
& a_{3}^{\frac{7}{2}, \frac{15}{2}}=-\frac{2}{1105}\left(-1105+\nu^{2}\right), \quad a_{5}^{\frac{7}{2}, \frac{15}{2}}=\frac{2}{139425}(-13+\nu)(13+\nu)\left(-825+\nu^{2}\right), \\
& a_{1}^{\frac{9}{2}, \frac{3}{2}}=2, \quad a_{1}^{\frac{9}{2}, \frac{5}{2}}=2, \quad a_{3}^{\frac{9}{2}, \frac{5}{2}}=-\frac{2}{189}\left(-189+\nu^{2}\right), \quad a_{1}^{\frac{9}{2}, \frac{7}{2}}=2, \\
& a_{3}^{\frac{9}{2}, \frac{7}{2}}=-\frac{2}{385}\left(-385+\nu^{2}\right), \quad a_{5}^{\frac{9}{2}, \frac{7}{2}}=\frac{2}{11025}(-15+\nu)(-7+\nu)(7+\nu)(15+\nu), \\
& a_{1}^{\frac{9}{2}, \frac{9}{2}}=2, \quad a_{3}^{\frac{9}{2}, \frac{9}{2}}=-\frac{2}{637}\left(-637+\nu^{2}\right), \quad a_{5}^{\frac{9}{2}, \frac{9}{2}}=\frac{2}{40425}\left(40425-554 \nu^{2}+\nu^{4}\right),
\end{aligned}
$$




$$
\begin{aligned}
& a_{7}^{\frac{9}{2}, \frac{9}{2}}=-\frac{2}{231525}(-7+\nu)(-5+\nu)(5+\nu)(7+\nu)\left(-189+\nu^{2}\right), \quad a_{1}^{\frac{9}{2}, \frac{11}{2}}=2, \\
& a_{3}^{\frac{9}{2}, \frac{11}{2}}=-\frac{2}{945}\left(-945+\nu^{2}\right), \quad a_{5}^{\frac{9}{2}, \frac{11}{2}}=\frac{2}{105105}\left(105105-914 \nu^{2}+\nu^{4}\right), \\
& a_{7}^{\frac{9}{2}, \frac{11}{2}}=-\frac{2}{1528065}(-9+\nu)(-7+\nu)(7+\nu)(9+\nu)\left(-385+\nu^{2}\right), \quad a_{1}^{\frac{9}{2}, \frac{13}{2}}=2, \\
& a_{3}^{\frac{9}{2}, \frac{13}{2}}=-\frac{2}{1309}\left(-1309+\nu^{2}\right), \quad a_{5}^{\frac{9}{2}, \frac{13}{2}}=\frac{2}{225225}\left(225225-1354 \nu^{2}+\nu^{4}\right), \\
& a_{7}^{\frac{9}{2}, \frac{13}{2}}=-\frac{2}{6243237}(-11+\nu)(-9+\nu)(9+\nu)(11+\nu)\left(-637+\nu^{2}\right), \quad a_{1}^{\frac{11}{2}, \frac{3}{2}}=2, \\
& a_{1}^{\frac{11}{2}, \frac{5}{2}}=2, \quad a_{3}^{\frac{11}{2}, \frac{5}{2}}=-\frac{2}{297}\left(-297+\nu^{2}\right), \quad a_{1}^{\frac{11}{2}, \frac{7}{2}}=2, a_{3}^{\frac{11}{2}, \frac{7}{2}}=-\frac{2}{585}\left(-585+\nu^{2}\right), \\
& a_{5}^{\frac{11}{2}, \frac{7}{2}}=\frac{2}{31185}(-9+\nu)(9+\nu)\left(-385+\nu^{2}\right), \quad a_{1}^{\frac{11}{2}, \frac{9}{2}}=2, \\
& a_{3}^{\frac{11}{2}, \frac{9}{2}}=-\frac{2}{945}\left(-945+\nu^{2}\right), \quad a_{5}^{\frac{11}{2}, \frac{9}{2}}=\frac{2}{105105}\left(105105-914 \nu^{2}+\nu^{4}\right), \\
& a_{7}^{\frac{11}{2}, \frac{9}{2}}=-\frac{2}{1528065}(-9+\nu)(-7+\nu)(7+\nu)(9+\nu)\left(-385+\nu^{2}\right), \quad a_{1}^{\frac{11}{2}, \frac{11}{2}}=2, \\
& a_{3}^{\frac{11}{2}, \frac{11}{2}}=-\frac{2}{1377}\left(-1377+\nu^{2}\right), \quad a_{5}^{\frac{11}{2}, \frac{11}{2}}=\frac{2}{257985}\left(257985-1474 \nu^{2}+\nu^{4}\right), \\
& a_{7}^{\frac{11}{2}, \frac{11}{2}}=-\frac{2}{8513505}(-9+\nu)(9+\nu)\left(105105-914 \nu^{2}+\nu^{4}\right), \\
& a_{9}^{\frac{11}{2}, \frac{11}{2}}=\frac{2}{29469825}(-9+\nu)(-7+\nu)(-5+\nu)(5+\nu)(7+\nu)(9+\nu)\left(-297+\nu^{2}\right), \\
& a_{1}^{\frac{13}{2}, \frac{3}{2}}=2, \quad a_{1}^{\frac{13}{2}, \frac{5}{2}}=2, \quad a_{3}^{\frac{13}{2}, \frac{5}{2}}=-\frac{2}{429}\left(-429+\nu^{2}\right), \quad a_{1}^{\frac{13}{2}, \frac{7}{2}}=2, \\
& a_{3}^{\frac{13}{2}, \frac{7}{2}}=-\frac{2}{825}\left(-825+\nu^{2}\right), \quad a_{5}^{\frac{13}{2}, \frac{7}{2}}=\frac{2}{70785}(-11+\nu)(11+\nu)\left(-585+\nu^{2}\right), \\
& a_{1}^{\frac{13}{2}, \frac{9}{2}}=2, \quad a_{3}^{\frac{13}{2}, \frac{9}{2}}=-\frac{2}{1309}\left(-1309+\nu^{2}\right), \\
& a_{5}^{\frac{13}{2}, \frac{9}{2}}=\frac{2}{225225}\left(225225-1354 \nu^{2}+\nu^{4}\right) \text {, } \\
& a_{7}^{\frac{13}{2}, \frac{9}{2}}=-\frac{2}{6243237}(-11+\nu)(-9+\nu)(9+\nu)(11+\nu)\left(-637+\nu^{2}\right), \quad a_{1}^{\frac{15}{2}, \frac{3}{2}}=2, \\
& a_{1}^{\frac{15}{2}, \frac{5}{2}}=2, \quad a_{3}^{\frac{15}{2}, \frac{5}{2}}=-\frac{2}{585}\left(-585+\nu^{2}\right), \quad a_{1}^{\frac{15}{2}, \frac{7}{2}}=2, \\
& a_{3}^{\frac{15}{2}, \frac{7}{2}}=-\frac{2}{1105}\left(-1105+\nu^{2}\right), \quad a_{5}^{\frac{15}{2}, \frac{7}{2}}=\frac{2}{139425}(-13+\nu)(13+\nu)\left(-825+\nu^{2}\right), \\
& a_{1}^{\frac{17}{2}, \frac{3}{2}}=2, \quad a_{1}^{\frac{17}{2}, \frac{5}{2}}=2, \quad a_{3}^{\frac{17}{2}, \frac{5}{2}}=-\frac{2}{765}\left(-765+\nu^{2}\right), \quad a_{1}^{\frac{19}{2}, \frac{3}{2}}=2 .
\end{aligned}
$$

The first nontrivial $\nu$ dependence appears in $a_{3}^{\frac{5}{2}, \frac{5}{2}}$. From the previous experience, we expect that there is a linear $\nu$ dependence in the generalized hypergeometric functions. By adding the two of them, we obtain the above coefficient which has the constant term as well as the quadratic term in $\nu$. The first nontrivial quartic term in $\nu$ dependence appears in $a_{5}^{\frac{7}{2}, \frac{7}{2}}$. There will be a linear term and the third order term in $\nu$ dependence in the generalized hypergeometric functions. But it is nontrivial to obtain their explicit forms. We observe that there are sextic term in the coefficient $a_{7}^{\frac{9}{2}, \frac{9}{2}}$ and octic term in the coefficient $a_{9}^{\frac{11}{2}, \frac{11}{2}}$. 
We should determine the odd power of $\nu$ terms in the generalized hypergeometric functions before we add them. ${ }^{21}$

The other structure constants can be obtained as follows:

$$
\begin{aligned}
& b_{3}^{\frac{5}{2}, \frac{5}{2}}=-\frac{4 \nu}{15}, \quad b_{3}^{\frac{5}{2}, \frac{7}{2}}=-\frac{4 \nu}{35}, \quad b_{3}^{\frac{5}{2}, \frac{9}{2}}=-\frac{4 \nu}{63}, \quad b_{3}^{\frac{5}{2}, \frac{11}{2}}=-\frac{4 \nu}{99}, b_{3}^{\frac{5}{2}, \frac{13}{2}}=-\frac{4 \nu}{143}, \\
& b_{3}^{\frac{5}{2}, \frac{15}{2}}=-\frac{4 \nu}{195}, \quad b_{3}^{\frac{5}{2}, \frac{17}{2}}=-\frac{4 \nu}{255}, \quad b_{3}^{\frac{7}{2}, \frac{5}{2}}=-\frac{4 \nu}{35}, \quad b_{3}^{\frac{7}{2}, \frac{7}{2}}=-\frac{4 \nu}{75}, \\
& b_{5}^{\frac{7}{2}, \frac{7}{2}}=\frac{8}{525}(-5+\nu) \nu(5+\nu), \quad b_{3}^{\frac{7}{2}, \frac{9}{2}}=-\frac{12 \nu}{385}, \quad b_{5}^{\frac{7}{2}, \frac{9}{2}}=\frac{8}{2205}(-7+\nu) \nu(7+\nu), \\
& b_{3}^{\frac{7}{2},}, \frac{11}{2}=-\frac{4 \nu}{195}, \quad b_{5}^{\frac{7}{2}, \frac{11}{2}}=\frac{8}{6237}(-9+\nu) \nu(9+\nu), \quad b_{3}^{\frac{7}{2}, \frac{13}{2}}=-\frac{4 \nu}{275}, \\
& b_{5}^{\frac{7}{2}, \frac{13}{2}}=\frac{8}{14157}(-11+\nu) \nu(11+\nu), \quad b_{3}^{\frac{7}{2}, \frac{15}{2}}=-\frac{12 \nu}{1105}, \\
& b_{5}^{\frac{7}{2}, \frac{15}{2}}=\frac{8}{27885}(-13+\nu) \nu(13+\nu), \quad b_{3}^{\frac{9}{2}, \frac{5}{2}}=-\frac{4 \nu}{63}, \quad b_{3}^{\frac{9}{2}, \frac{7}{2}}=-\frac{12 \nu}{385}, \\
& b_{5}^{\frac{9}{2}, \frac{7}{2}}=\frac{8}{2205}(-7+\nu) \nu(7+\nu), \quad b_{3}^{\frac{9}{2}, \frac{9}{2}}=-\frac{12 \nu}{637}, \quad b_{5}^{\frac{9}{2}, \frac{9}{2}}=\frac{8}{24255} \nu\left(-287+3 \nu^{2}\right), \\
& b_{7}^{\frac{9}{2}, \frac{9}{2}}=-\frac{4}{11025}(-7+\nu)(-5+\nu) \nu(5+\nu)(7+\nu), \quad b_{3}^{\frac{9}{2}, \frac{11}{2}}=-\frac{4 \nu}{315}, \\
& b_{5}^{\frac{9}{2}, \frac{11}{2}}=\frac{8}{63063} \nu\left(-467+3 \nu^{2}\right), \quad b_{7}^{\frac{9}{2}, \frac{11}{2}}=-\frac{4}{72765}(-9+\nu)(-7+\nu) \nu(7+\nu)(9+\nu), \\
& b_{3}^{\frac{9}{2}, \frac{13}{2}}=-\frac{12 \nu}{1309}, \quad b_{5}^{\frac{9}{2}, \frac{13}{2}}=\frac{8}{45045} \nu\left(-229+\nu^{2}\right), \\
& b_{7}^{\frac{9}{2}, \frac{13}{2}}=-\frac{4}{297297}(-11+\nu)(-9+\nu) \nu(9+\nu)(11+\nu), \quad b_{3}^{\frac{11}{2}, \frac{5}{2}}=-\frac{4 \nu}{99}, \\
& b_{3}^{\frac{11}{2}, \frac{7}{2}}=-\frac{4 \nu}{195}, \quad b_{5}^{\frac{11}{2}, \frac{7}{2}}=\frac{8}{6237}(-9+\nu) \nu(9+\nu), \quad b_{3}^{\frac{11}{2}, \frac{9}{2}}=-\frac{4 \nu}{315}, \\
& b_{5}^{\frac{11}{2}, \frac{9}{2}}=\frac{8}{63063} \nu\left(-467+3 \nu^{2}\right), \quad b_{7}^{\frac{11}{2}, \frac{9}{2}}=-\frac{4}{72765}(-9+\nu)(-7+\nu) \nu(7+\nu)(9+\nu), \\
& b_{3}^{\frac{11}{2}, \frac{11}{2}}=-\frac{4 \nu}{459}, \quad b_{5}^{\frac{11}{2}, \frac{11}{2}}=\frac{8}{51597} \nu\left(-249+\nu^{2}\right), \\
& b_{7}^{\frac{11}{2}, \frac{11}{2}}=-\frac{4}{1216215}(-9+\nu) \nu(9+\nu)\left(-467+3 \nu^{2}\right) \text {, } \\
& b_{9}^{\frac{11}{2}, \frac{11}{2}}=\frac{16}{3274425}(-9+\nu)(-7+\nu)(-5+\nu) \nu(5+\nu)(7+\nu)(9+\nu), b_{3}^{\frac{13}{2}, \frac{5}{2}}=-\frac{4 \nu}{143} \text {, } \\
& b_{3}^{\frac{13}{2}, \frac{7}{2}}=-\frac{4 \nu}{275}, \quad b_{5}^{\frac{13}{2}, \frac{7}{2}}=\frac{8}{14157}(-11+\nu) \nu(11+\nu), b_{3}^{\frac{13}{2}, \frac{9}{2}}=-\frac{12 \nu}{1309}, \\
& b_{5}^{\frac{13}{2}, \frac{9}{2}}=\frac{8}{45045} \nu\left(-229+\nu^{2}\right), b_{7}^{\frac{13}{2}, \frac{9}{2}}=-\frac{4}{297297}(-11+\nu)(-9+\nu) \nu(9+\nu)(11+\nu) \text {, } \\
& b_{3}^{\frac{15}{2}, \frac{5}{2}}=-\frac{4 \nu}{195}, \quad b_{3}^{\frac{15}{2}, \frac{7}{2}}=-\frac{12 \nu}{1105}, \quad b_{5}^{\frac{15}{2}, \frac{7}{2}}=\frac{8}{27885}(-13+\nu) \nu(13+\nu), \\
& b_{3}^{\frac{17}{2}, \frac{5}{2}}=-\frac{4 \nu}{255} \text {. }
\end{aligned}
$$

\footnotetext{
${ }^{21} \mathrm{As}$ found in the footnote 20 , we obtain, for the second argument 2 of generalized hypergeometric function, $\phi_{4}^{3,3}(\mu, 2)=\frac{1}{45}\left(45-2 \nu-\nu^{2}\right), \phi_{6}^{4,4}(\mu, 2)=\frac{1}{2625}\left(2625-268 \nu-130 \nu^{2}+4 \nu^{3}+\nu^{4}\right)$ and $\phi_{8}^{5,5}(\mu, 2)=$ $\frac{1}{231525}\left(231525-32622 \nu-15211 \nu^{2}+1092 \nu^{3}+263 \nu^{4}-6 \nu^{5}-\nu^{6}\right)$. For example, we can check that $\phi_{4}^{3,3}(\mu, 2)$ looks similar to $\phi_{4}^{3,3}(\mu, 1)$ in the sense that the three lower elements are the same while the one upper element is different from each other. Because the fourth upper element in the former is the same as the third upper element in the latter, the number of terms in the generalized hypergeometric functions is the same with different coefficients. There are odd $\nu$ terms in the above expressions. By taking $\nu \rightarrow-\nu$, we obtain the corresponding generalized hypergeometric functions and as done before, we obtain the coefficients $a_{3}^{\frac{5}{2}, \frac{5}{2}}, a_{5}^{\frac{7}{2}, \frac{7}{2}}$ and $a_{7}^{\frac{9}{2}, \frac{9}{2}}$ respectively.
} 
It is easy to observe that some of the coefficients in eq. (C.11) are equal to the ones in (3.12). Let us focus on the cubic term in $\nu$ : the coefficient $b_{5}^{\frac{7}{2}, \frac{7}{2}}$ in eq. (C.11) is the same as $b_{6}^{4,4}$ in (3.12). Then we can try to determine the explicit form for the generalized hypergeometric function by following the procedure around (3.12). The corresponding quartic terms $(\nu \mp$ $3)(\nu \pm 5)(\nu \mp 5)(\nu \pm 7)$ for the above cubic term can arise in the generalized hypergeometric functions. Moreover, the sextic terms $(\nu \pm 3)(\nu \mp 5)(\nu \pm 5)(\nu \mp 7)(\nu \pm 7)(\nu \mp 9)$ for the above quintic term in eq. (C.11) can arise in the generalized hypergeometric functions. Similarly, the octic terms $(\nu \pm 3)(\nu \mp 5)(\nu \pm 5)(\nu \mp 7)(\nu \pm 7)(\nu \mp 9)(\nu \pm 9)(\nu \mp 11)$ for the above heptic term in eq. (C.11) can arise. We need to obtain the explicit forms, by trying to vary the various elements in order to match with the odd powers of $\nu$ in eq. (C.11), for the generalized hypergeometric functions.

Then we have the following relations from eqs. (C.10) and (C.11)

$$
\begin{aligned}
a_{2 r-1}^{h_{1}+\frac{1}{2}, h_{2}+\frac{1}{2}}(\mu)= & { }_{4} F_{3}\left[\begin{array}{c}
\frac{1}{2}+\mu, \frac{1}{2}-\mu, \frac{3-2 r}{2}, \frac{2-2 r}{2} \\
\frac{1}{2}-h_{1}, \frac{1}{2}-h_{2}, \frac{5}{2}+h_{1}+h_{2}-2 r \mid 1
\end{array} \mid 1\right] \\
& +{ }_{4} F_{3}\left[\begin{array}{c}
\frac{3}{2}-\mu,-\frac{1}{2}+\mu, \frac{3-2 r}{2}, \frac{2-2 r}{2} \\
\frac{1}{2}-h_{1}, \frac{1}{2}-h_{2}, \frac{5}{2}+h_{1}+h_{2}-2 r
\end{array} \mid 1\right], \\
b_{2 r-1}^{h_{1}+\frac{1}{2}, h_{2}+\frac{1}{2}}(\mu)= & { }_{4} F_{3}\left[\begin{array}{c}
\frac{1}{2}+\mu, \frac{1}{2}-\mu, \frac{2-2 r}{2}, \frac{1-2 r}{2} \\
\frac{1}{2}-h_{1}, \frac{1}{2}-h_{2}, \frac{5}{2}+h_{1}+h_{2}-2 r
\end{array} \mid 1\right] \\
& -{ }_{4} F_{3}\left[\begin{array}{c}
\frac{3}{2}-\mu,-\frac{1}{2}+\mu, \frac{2-2 r}{2}, \frac{1-2 r}{2} \\
\frac{1}{2}-h_{1}, \frac{1}{2}-h_{2}, \frac{5}{2}+h_{1}+h_{2}-2 r
\end{array} \mid 1\right] .
\end{aligned}
$$

The structure constant $a_{2 r-1}^{h_{1}+\frac{1}{2}, h_{2}+\frac{1}{2}}$ is not "saalschutzian" type while the structure constant $b_{2 r-1}^{h_{1}+\frac{1}{2}, h_{2}+\frac{1}{2}}$ is "saalschutzian" type.

We realize that after collecting $\left(h_{1}+1\right)$ and $\left(h_{2}+1\right)$ in the right hand side of the first relation of eq. (C.12), we obtain the first relation of (3.13) by a shift $\frac{1}{2}$ in the upper third and fourth elements of generalized hypergeometric function. Similarly, after collecting $\left(h_{1}+1\right)$ and $\left(h_{2}+1\right)$ in the right hand side of the second relation of eq. (C.12), we obtain the second relation of (3.13). This observation is encoded in (4.1). We will see that the above two structure constants in eq. (C.12) are denoted by the third one in (4.2).

\section{C.2.2 Operator $K$ dependence in the oscillators}

We can analyze the other case corresponding to (4.9) as follows. By introducing the $\nu$ dependent structure constants, we have

$$
\begin{aligned}
& \{\underbrace{\hat{y}_{(1} \ldots \hat{y}_{1}}_{h_{1}-\frac{1}{2}+\rho} \underbrace{\left.\hat{y}_{2} \ldots \hat{y}_{2}\right)}_{h_{1}-\frac{1}{2}-\rho}, \underbrace{\hat{y}_{(1} \ldots \hat{y}_{1}}_{h_{2}-\frac{1}{2}+\omega} \underbrace{\left.\hat{y}_{2} \ldots \hat{y}_{2}\right)}_{h_{2}-\frac{1}{2}-\omega} K\}=\sum_{r=1}^{\left[\frac{h_{1}+h_{2}}{2}\right]} \mathrm{i}(-1)^{r} N_{2 r}^{h_{1}+\frac{1}{2} h_{2}+\frac{1}{2}}(\rho, \omega) \\
& \times[-\frac{1}{(2 r-1) !} a_{2 r}^{h_{1}+\frac{1}{2}, h_{2}+\frac{1}{2}}(\nu) \underbrace{\hat{y}_{(1} \ldots \ldots \ldots \hat{y}_{1}}_{h_{1}+h_{2}-2 r+\rho+\omega} \underbrace{\left.\hat{y}_{2} \ldots \ldots \ldots \hat{y}_{2}\right)}_{h_{1}+h_{2}-2 r-\rho-\omega} K \\
& +\frac{2\left(h_{1}+h_{2}-r\right)+1}{(2 r) !} b_{2 r}^{h_{1}+\frac{1}{2}, h_{2}+\frac{1}{2}}(\nu) \underbrace{\hat{y}_{(1} \ldots \ldots \ldots \hat{y}_{1}}_{h_{1}+h_{2}-2 r+\rho+\omega} \underbrace{\hat{y}_{2} \ldots \ldots \ldots \hat{y}_{2)}}_{h_{1}+h_{2}-2 r-\rho-\omega}] .
\end{aligned}
$$


It turns out that from the analysis of eq. (C.13)

$$
\begin{aligned}
& a_{2}^{\frac{3}{2}, \frac{3}{2}}=2, \quad a_{2}^{\frac{3}{2}, \frac{5}{2}}=2, \quad a_{2}^{\frac{3}{2}, \frac{7}{2}}=2, \quad a_{2}^{\frac{3}{2}, \frac{9}{2}}=2, \quad a_{2}^{\frac{3}{2}, \frac{11}{2}}=2, \quad a_{2}^{\frac{3}{2}, \frac{13}{2}}=2, \\
& a_{2}^{\frac{3}{2}, \frac{15}{2}}=2, \quad a_{2}^{\frac{3}{2}, \frac{17}{2}}=2, \quad a_{2}^{\frac{3}{2}, \frac{19}{2}}=2, \quad a_{2}^{\frac{5}{2}, \frac{3}{2}}=2, \quad a_{2}^{\frac{5}{2}, \frac{5}{2}}=2, \\
& a_{4}^{\frac{5}{2}, \frac{5}{2}}=-\frac{2}{9}(-3+\nu)(3+\nu), \quad a_{2}^{\frac{5}{2}, \frac{7}{2}}=2, \quad a_{4}^{\frac{5}{2}, \frac{7}{2}}=-\frac{2}{25}(-5+\nu)(5+\nu), \\
& a_{2}^{\frac{5}{2}, \frac{9}{2}}=2, \quad a_{4}^{\frac{5}{2}, \frac{9}{2}}=-\frac{2}{49}(-7+\nu)(7+\nu), \quad a_{2}^{\frac{5}{2}, \frac{11}{2}}=2, \\
& a_{4}^{\frac{5}{2}, \frac{11}{2}}=-\frac{2}{81}(-9+\nu)(9+\nu), \quad a_{2}^{\frac{5}{2}, \frac{13}{2}}=2, \quad a_{4}^{\frac{5}{2}, \frac{13}{2}}=-\frac{2}{121}(-11+\nu)(11+\nu), \\
& a_{2}^{\frac{5}{2}, \frac{15}{2}}=2, \quad a_{4}^{\frac{5}{2}, \frac{15}{2}}=-\frac{2}{169}(-13+\nu)(13+\nu), \quad a_{2}^{\frac{5}{2}, \frac{17}{2}}=2, \\
& a_{4}^{\frac{5}{2}, \frac{17}{2}}=-\frac{2}{225}(-15+\nu)(15+\nu), \quad a_{2}^{\frac{7}{2}, \frac{3}{2}}=2, \quad a_{2}^{\frac{7}{2}, \frac{5}{2}}=2, \\
& a_{4}^{\frac{7}{2}, \frac{5}{2}}=-\frac{2}{25}(-5+\nu)(5+\nu), \quad a_{2}^{\frac{7}{2}, \frac{7}{2}}=2, \quad a_{4}^{\frac{7}{2}, \frac{7}{2}}=-\frac{2}{175}\left(-175+3 \nu^{2}\right), \\
& a_{6}^{\frac{7}{2}, \frac{7}{2}}=\frac{2}{225}(-5+\nu)(-3+\nu)(3+\nu)(5+\nu), \quad a_{2}^{\frac{7}{2}, \frac{9}{2}}=2, \\
& a_{4}^{\frac{7}{2}, \frac{9}{2}}=-\frac{2}{105}\left(-105+\nu^{2}\right), \quad a_{6}^{\frac{7}{2}, \frac{9}{2}}=\frac{2}{1225}(-7+\nu)(-5+\nu)(5+\nu)(7+\nu), \\
& a_{2}^{\frac{7}{2}, \frac{11}{2}}=2, \quad a_{4}^{\frac{7}{2}, \frac{11}{2}}=-\frac{2}{165}\left(-165+\nu^{2}\right), \\
& a_{6}^{\frac{7}{2}, \frac{11}{2}}=\frac{2}{3969}(-9+\nu)(-7+\nu)(7+\nu)(9+\nu), \quad a_{2}^{\frac{7}{2}, \frac{13}{2}}=2, \\
& a_{4}^{\frac{7}{2}, \frac{13}{2}}=-\frac{2}{715}\left(-715+3 \nu^{2}\right), \quad a_{6}^{\frac{7}{2}, \frac{13}{2}}=\frac{2}{9801}(-11+\nu)(-9+\nu)(9+\nu)(11+\nu), \\
& a_{2}^{\frac{7}{2}, \frac{15}{2}}=2, \quad a_{4}^{\frac{7}{2}, \frac{15}{2}}=-\frac{2}{325}\left(-325+\nu^{2}\right), \\
& a_{6}^{\frac{7}{2}, \frac{15}{2}}=\frac{2}{20449}(-13+\nu)(-11+\nu)(11+\nu)(13+\nu), \quad a_{2}^{\frac{9}{2}, \frac{3}{2}}=2, \quad a_{2}^{\frac{9}{2}, \frac{5}{2}}=2, \\
& a_{4}^{\frac{9}{2}, \frac{5}{2}}=-\frac{2}{49}(-7+\nu)(7+\nu), \quad a_{2}^{\frac{9}{2}, \frac{7}{2}}=2, \quad a_{4}^{\frac{9}{2}, \frac{7}{2}}=-\frac{2}{105}\left(-105+\nu^{2}\right), \\
& a_{6}^{\frac{9}{2}, \frac{7}{2}}=\frac{2}{1225}(-7+\nu)(-5+\nu)(5+\nu)(7+\nu), \quad a_{2}^{\frac{9}{2}, \frac{9}{2}}=2, \\
& a_{4}^{\frac{9}{2}, \frac{9}{2}}=-\frac{2}{539}\left(-539+3 \nu^{2}\right), \quad a_{6}^{\frac{9}{2}, \frac{9}{2}}=\frac{2}{5145}(-7+\nu)(7+\nu)\left(-105+\nu^{2}\right), \\
& a_{8}^{\frac{9}{2}, \frac{9}{2}}=-\frac{2}{11025}(-7+\nu)(-5+\nu)(-3+\nu)(3+\nu)(5+\nu)(7+\nu), \quad a_{2}^{\frac{9}{2}, \frac{11}{2}}=2, \\
& a_{4}^{\frac{9}{2}, \frac{11}{2}}=-\frac{2}{273}\left(-273+\nu^{2}\right), \quad a_{6}^{\frac{9}{2}, \frac{11}{2}}=\frac{2}{43659}(-9+\nu)(9+\nu)\left(-539+3 \nu^{2}\right), \\
& a_{8}^{\frac{9}{2}, \frac{11}{2}}=-\frac{2}{99225}(-9+\nu)(-7+\nu)(-5+\nu)(5+\nu)(7+\nu)(9+\nu), \quad a_{2}^{\frac{9}{2}, \frac{13}{2}}=2, \\
& a_{4}^{\frac{9}{2}, \frac{13}{2}}=-\frac{2}{385}\left(-385+\nu^{2}\right), \quad a_{6}^{\frac{9}{2}, \frac{13}{2}}=\frac{2}{33033}(-11+\nu)(11+\nu)\left(-273+\nu^{2}\right), \\
& a_{8}^{\frac{9}{2}, \frac{13}{2}}=-\frac{2}{480249}(-11+\nu)(-9+\nu)(-7+\nu)(7+\nu)(9+\nu)(11+\nu), \quad a_{2}^{\frac{11}{2}, \frac{3}{2}}=2 \text {, } \\
& a_{2}^{\frac{11}{2}, \frac{5}{2}}=2, \quad a_{4}^{\frac{11}{2}, \frac{5}{2}}=-\frac{2}{81}(-9+\nu)(9+\nu), \quad a_{2}^{\frac{11}{2}, \frac{7}{2}}=2,
\end{aligned}
$$




$$
\begin{aligned}
& a_{4}^{\frac{11}{2}, \frac{7}{2}}=-\frac{2}{165}\left(-165+\nu^{2}\right), \quad a_{6}^{\frac{11}{2}, \frac{7}{2}}=\frac{2}{3969}(-9+\nu)(-7+\nu)(7+\nu)(9+\nu), \\
& a_{2}^{\frac{11}{2}, \frac{9}{2}}=2, \quad a_{4}^{\frac{11}{2}, \frac{9}{2}}=-\frac{2}{273}\left(-273+\nu^{2}\right), \\
& a_{6}^{\frac{11}{2}, \frac{9}{2}}=\frac{2}{43659}(-9+\nu)(9+\nu)\left(-539+3 \nu^{2}\right), \\
& a_{8}^{\frac{11}{2}, \frac{9}{2}}=-\frac{2}{99225}(-9+\nu)(-7+\nu)(-5+\nu)(5+\nu)(7+\nu)(9+\nu), \quad a_{2}^{\frac{11}{2}, \frac{11}{2}}=2, \\
& a_{4}^{\frac{11}{2}, \frac{11}{2}}=-\frac{2}{405}\left(-405+\nu^{2}\right), \quad a_{6}^{\frac{11}{2}, \frac{11}{2}}=\frac{2}{567567}\left(567567-6430 \nu^{2}+15 \nu^{4}\right), \\
& a_{8}^{\frac{11}{2}, \frac{11}{2}}=-\frac{2}{654885}(-9+\nu)(-7+\nu)(7+\nu)(9+\nu)\left(-165+\nu^{2}\right), \\
& a_{10}^{\frac{11}{2}, \frac{11}{2}}=\frac{2}{893025}(-9+\nu)(-7+\nu)(-5+\nu)(-3+\nu)(3+\nu)(5+\nu)(7+\nu)(9+\nu), \\
& a_{2}^{\frac{13}{2}, \frac{3}{2}}=2, \quad a_{2}^{\frac{13}{2}, \frac{5}{2}}=2, \quad a_{4}^{\frac{13}{2}, \frac{5}{2}}=-\frac{2}{121}(-11+\nu)(11+\nu), \quad a_{2}^{\frac{13}{2}, \frac{7}{2}}=2, \\
& a_{4}^{\frac{13}{2}, \frac{7}{2}}=-\frac{2}{715}\left(-715+3 \nu^{2}\right), \quad a_{6}^{\frac{13}{2}, \frac{7}{2}}=\frac{2}{9801}(-11+\nu)(-9+\nu)(9+\nu)(11+\nu), \\
& a_{2}^{\frac{13}{2}, \frac{9}{2}}=2, \quad a_{4}^{\frac{13}{2}, \frac{9}{2}}=-\frac{2}{385}\left(-385+\nu^{2}\right), \\
& a_{6}^{\frac{13}{2}, \frac{9}{2}}=\frac{2}{33033}(-11+\nu)(11+\nu)\left(-273+\nu^{2}\right), \\
& a_{8}^{\frac{13}{2}, \frac{9}{2}}=-\frac{2}{480249}(-11+\nu)(-9+\nu)(-7+\nu)(7+\nu)(9+\nu)(11+\nu), \quad a_{2}^{\frac{15}{2}, \frac{3}{2}}=2, \\
& a_{2}^{\frac{15}{2}, \frac{5}{2}}=2, \quad a_{4}^{\frac{15}{2}, \frac{5}{2}}=-\frac{2}{169}(-13+\nu)(13+\nu), \quad a_{2}^{\frac{15}{2}, \frac{7}{2}}=2, \\
& a_{4}^{\frac{15}{2}, \frac{7}{2}}=-\frac{2}{325}\left(-325+\nu^{2}\right), \quad a_{6}^{\frac{15}{2}, \frac{7}{2}}=\frac{2}{20449}(-13+\nu)(-11+\nu)(11+\nu)(13+\nu), \\
& a_{2}^{\frac{17}{2}, \frac{3}{2}}=2, \quad a_{2}^{\frac{17}{2}, \frac{5}{2}}=2, \quad a_{4}^{\frac{17}{2}, \frac{5}{2}}=-\frac{2}{225}(-15+\nu)(15+\nu), a_{2}^{\frac{19}{2}, \frac{3}{2}}=2 .
\end{aligned}
$$

Some of the coefficients in eq. (C.14) overlap with the ones in eq. (C.2). For example, the coefficient $a_{4}^{\frac{5}{2}, \frac{7}{2}}$ of eq. (C.14) is exactly the same as the coefficient $a_{4}^{3, \frac{7}{2}}$ of eq. (C.2). Note that there is a shift in $h_{1}$. The explicit $\nu$ dependence in the generalized hypergeometric functions can be determined similarly.

Furthermore we have

$$
\begin{aligned}
& b_{2}^{\frac{3}{2},}, \frac{3}{2}=-\frac{4 \nu}{3}, \quad b_{2}^{\frac{3}{2}, \frac{5}{2}}=-\frac{4 \nu}{15}, \quad b_{2}^{\frac{3}{2},}, \frac{7}{2}=-\frac{4 \nu}{35}, \quad b_{2}^{\frac{3}{2}, \frac{9}{2}}=-\frac{4 \nu}{63}, \quad b_{2}^{\frac{3}{2}, \frac{11}{2}}=-\frac{4 \nu}{99}, \\
& b_{2}^{\frac{3}{2}}, \frac{13}{2}=-\frac{4 \nu}{143}, \quad b_{2}^{\frac{3}{2}, \frac{15}{2}}=-\frac{4 \nu}{195}, \quad b_{2}^{\frac{3}{2}, \frac{17}{2}}=-\frac{4 \nu}{255}, \quad b_{2}^{\frac{3}{2}, \frac{19}{2}}=-\frac{4 \nu}{323}, \\
& b_{2}^{\frac{5}{2}, \frac{3}{2}}=-\frac{4 \nu}{15}, \quad b_{2}^{\frac{5}{2}, \frac{5}{2}}=-\frac{4 \nu}{63}, \quad b_{4}^{\frac{5}{2}, \frac{5}{2}}=\frac{8}{45}(-3+\nu) \nu(3+\nu), \quad b_{2}^{\frac{5}{2}, \frac{7}{2}}=-\frac{4 \nu}{135}, \\
& b_{4}^{\frac{5}{2}, \frac{7}{2}}=\frac{8}{525}(-5+\nu) \nu(5+\nu), \quad b_{2}^{\frac{5}{2}, \frac{9}{2}}=-\frac{4 \nu}{231}, \quad b_{4}^{\frac{5}{2}, \frac{9}{2}}=\frac{8}{2205}(-7+\nu) \nu(7+\nu), \\
& b_{2}^{\frac{5}{2},}, \frac{11}{2}=-\frac{4 \nu}{351}, \quad b_{4}^{\frac{5}{2}, \frac{11}{2}}=\frac{8}{6237}(-9+\nu) \nu(9+\nu), \quad b_{2}^{\frac{5}{2}, \frac{13}{2}}=-\frac{4 \nu}{495}, \\
& b_{4}^{\frac{5}{2}, \frac{13}{2}}=\frac{8}{14157}(-11+\nu) \nu(11+\nu), \quad b_{2}^{\frac{5}{2}, \frac{15}{2}}=-\frac{4 \nu}{663}, \\
& b_{4}^{\frac{5}{2}, \frac{15}{2}}=\frac{8}{27885}(-13+\nu) \nu(13+\nu), \quad b_{2}^{\frac{5}{2}, \frac{17}{2}}=-\frac{4 \nu}{855},
\end{aligned}
$$




$$
\begin{aligned}
& b_{4}^{\frac{5}{2}, \frac{17}{2}}=\frac{8}{49725}(-15+\nu) \nu(15+\nu), \quad b_{2}^{\frac{7}{2}, \frac{3}{2}}=-\frac{4 \nu}{35}, \quad b_{2}^{\frac{7}{2}, \frac{5}{2}}=-\frac{4 \nu}{135}, \\
& b_{4}^{\frac{7}{2}, \frac{5}{2}}=\frac{8}{525}(-5+\nu) \nu(5+\nu), \quad b_{2}^{\frac{7}{2}, \frac{7}{2}}=-\frac{4 \nu}{275}, \quad b_{4}^{\frac{7}{2}, \frac{7}{2}}=\frac{8}{4725} \nu\left(-85+\nu^{2}\right), \\
& b_{6}^{\frac{7}{2}, \frac{7}{2}}=-\frac{4}{525}(-5+\nu)(-3+\nu) \nu(3+\nu)(5+\nu), \quad b_{2}^{\frac{7}{2}, \frac{9}{2}}=-\frac{4 \nu}{455}, \\
& b_{4}^{\frac{7}{2}, \frac{9}{2}}=\frac{8}{17325}(-13+\nu) \nu(13+\nu), b_{6}^{\frac{7}{2}, \frac{9}{2}}=-\frac{4}{11025}(-7+\nu)(-5+\nu) \nu(5+\nu)(7+\nu) \text {, } \\
& b_{2}^{\frac{7}{2}, \frac{11}{2}}=-\frac{4 \nu}{675}, \quad b_{4}^{\frac{7}{2}, \frac{11}{2}}=\frac{8}{45045} \nu\left(-277+\nu^{2}\right), \\
& b_{6}^{\frac{7}{2}, \frac{11}{2}}=-\frac{4}{72765}(-9+\nu)(-7+\nu) \nu(7+\nu)(9+\nu), \quad b_{2}^{\frac{7}{2}, \frac{13}{2}}=-\frac{4 \nu}{935}, \\
& b_{4}^{\frac{7}{2}, \frac{13}{2}}=\frac{8}{96525} \nu\left(-409+\nu^{2}\right) \text {, } \\
& b_{6}^{\frac{7}{2}, \frac{13}{2}}=-\frac{4}{297297}(-11+\nu)(-9+\nu) \nu(9+\nu)(11+\nu), \quad b_{2}^{\frac{7}{2}, \frac{15}{2}}=-\frac{4 \nu}{1235}, \\
& b_{4}^{\frac{7}{2}, \frac{15}{2}}=\frac{8}{182325} \nu\left(-565+\nu^{2}\right) \text {, } \\
& b_{6}^{\frac{7}{2}, \frac{15}{2}}=-\frac{4}{920205}(-13+\nu)(-11+\nu) \nu(11+\nu)(13+\nu), \quad b_{2}^{\frac{9}{2}, \frac{3}{2}}=-\frac{4 \nu}{63}, \\
& b_{2}^{\frac{9}{2}, \frac{5}{2}}=-\frac{4 \nu}{231}, \quad b_{4}^{\frac{9}{2}, \frac{5}{2}}=\frac{8}{2205}(-7+\nu) \nu(7+\nu), \quad b_{2}^{\frac{9}{2}, \frac{7}{2}}=-\frac{4 \nu}{455}, \\
& b_{4}^{\frac{9}{2}, \frac{7}{2}}=\frac{8}{17325}(-13+\nu) \nu(13+\nu), b_{6}^{\frac{9}{2}, \frac{7}{2}}=-\frac{4}{11025}(-7+\nu)(-5+\nu) \nu(5+\nu)(7+\nu), \\
& b_{2}^{\frac{9}{2}, \frac{9}{2}}=-\frac{4 \nu}{735}, \quad b_{4}^{\frac{9}{2}, \frac{9}{2}}=\frac{24}{175175} \nu\left(-329+\nu^{2}\right), \\
& b_{6}^{\frac{9}{2}, \frac{9}{2}}=-\frac{4}{169785}(-13+\nu)(-7+\nu) \nu(7+\nu)(13+\nu), \\
& b_{8}^{\frac{9}{2}, \frac{9}{2}}=\frac{16}{99225}(-7+\nu)(-5+\nu)(-3+\nu) \nu(3+\nu)(5+\nu)(7+\nu), \quad b_{2}^{\frac{9}{2}, \frac{11}{2}}=-\frac{4 \nu}{1071}, \\
& b_{4}^{\frac{9}{2}, \frac{11}{2}}=\frac{8}{143325}(-23+\nu) \nu(23+\nu) \text {, } \\
& b_{6}^{\frac{9}{2}, \frac{11}{2}}=-\frac{4}{945945}(-9+\nu) \nu(9+\nu)\left(-329+\nu^{2}\right), \\
& b_{8}^{\frac{9}{2}, \frac{11}{2}}=\frac{16}{3274425}(-9+\nu)(-7+\nu)(-5+\nu) \nu(5+\nu)(7+\nu)(9+\nu), b_{2}^{\frac{9}{2}, \frac{13}{2}}=-\frac{4 \nu}{1463}, \\
& b_{4}^{\frac{9}{2}, \frac{13}{2}}=\frac{8}{294525} \nu\left(-769+\nu^{2}\right), \\
& b_{6}^{\frac{9}{2}, \frac{13}{2}}=-\frac{4}{3468465}(-23+\nu)(-11+\nu) \nu(11+\nu)(23+\nu), \\
& b_{8}^{\frac{9}{2}, \frac{13}{2}}=\frac{16}{31216185}(-11+\nu)(-9+\nu)(-7+\nu) \nu(7+\nu)(9+\nu)(11+\nu), b_{2}^{\frac{11}{2}, \frac{3}{2}}=-\frac{4 \nu}{99}, \\
& b_{2}^{\frac{11}{2}, \frac{5}{2}}=-\frac{4 \nu}{351}, \quad b_{4}^{\frac{11}{2}, \frac{5}{2}}=\frac{8}{6237}(-9+\nu) \nu(9+\nu), \quad b_{2}^{\frac{11}{2}, \frac{7}{2}}=-\frac{4 \nu}{675}, \\
& b_{4}^{\frac{11}{2}, \frac{7}{2}}=\frac{8}{45045} \nu\left(-277+\nu^{2}\right), \quad b_{6}^{\frac{11}{2}, \frac{7}{2}}=-\frac{4}{72765}(-9+\nu)(-7+\nu) \nu(7+\nu)(9+\nu), \\
& b_{2}^{\frac{11}{2}, \frac{9}{2}}=-\frac{4 \nu}{1071}, \quad b_{4}^{\frac{11}{2}, \frac{9}{2}}=\frac{8}{143325}(-23+\nu) \nu(23+\nu), \\
& b_{6}^{\frac{11}{2}, \frac{9}{2}}=-\frac{4}{945945}(-9+\nu) \nu(9+\nu)\left(-329+\nu^{2}\right) \text {, }
\end{aligned}
$$




$$
\begin{aligned}
& b_{8}^{\frac{11}{2}, \frac{9}{2}}=\frac{16}{3274425}(-9+\nu)(-7+\nu)(-5+\nu) \nu(5+\nu)(7+\nu)(9+\nu), b_{2}^{\frac{11}{2}, \frac{11}{2}}=-\frac{4 \nu}{1539}, \\
& b_{4}^{\frac{11}{2}, \frac{11}{2}}=\frac{8}{337365} \nu\left(-837+\nu^{2}\right), \quad b_{6}^{\frac{11}{2}, \frac{11}{2}}=-\frac{4}{4729725} \nu\left(-641+\nu^{2}\right)\left(-129+\nu^{2}\right), \\
& b_{8}^{\frac{11}{2}, \frac{11}{2}}=\frac{16}{76621545}(-9+\nu)(-7+\nu) \nu(7+\nu)(9+\nu)\left(-277+\nu^{2}\right), \\
& b_{10}^{\frac{11}{2}, \frac{11}{2}}=-\frac{4}{1964655}(-9+\nu)(-7+\nu)(-5+\nu)(-3+\nu) \nu(3+\nu)(5+\nu)(7+\nu)(9+\nu), \\
& b_{2}^{\frac{13}{2}, \frac{3}{2}}=-\frac{4 \nu}{143}, \quad b_{2}^{\frac{13}{2}, \frac{5}{2}}=-\frac{4 \nu}{495}, \quad b_{4}^{\frac{13}{2}, \frac{5}{2}}=\frac{8}{14157}(-11+\nu) \nu(11+\nu), \\
& b_{2}^{\frac{13}{2}, \frac{7}{2}}=-\frac{4 \nu}{935}, \quad b_{4}^{\frac{13}{2}, \frac{7}{2}}=\frac{8}{96525} \nu\left(-409+\nu^{2}\right), \\
& b_{6}^{\frac{13}{2}}, \frac{7}{2}=-\frac{4}{297297}(-11+\nu)(-9+\nu) \nu(9+\nu)(11+\nu), \quad b_{2}^{\frac{13}{2}, \frac{9}{2}}=-\frac{4 \nu}{1463}, \\
& b_{4}^{\frac{13}{2}, \frac{9}{2}}=\frac{8}{294525} \nu\left(-769+\nu^{2}\right), \\
& b_{6}^{\frac{13}{2}, \frac{9}{2}}=-\frac{4}{3468465}(-23+\nu)(-11+\nu) \nu(11+\nu)(23+\nu), \\
& b_{8}^{\frac{13}{2}, \frac{9}{2}}=\frac{16}{31216185}(-11+\nu)(-9+\nu)(-7+\nu) \nu(7+\nu)(9+\nu)(11+\nu), \\
& b_{2}^{\frac{15}{2}, \frac{3}{2}}=-\frac{4 \nu}{195}, \quad b_{2}^{\frac{15}{2}, \frac{5}{2}}=-\frac{4 \nu}{663}, \quad b_{4}^{\frac{15}{2}, \frac{5}{2}}=\frac{8}{27885}(-13+\nu) \nu(13+\nu), \\
& b_{2}^{\frac{15}{2}, \frac{7}{2}}=-\frac{4 \nu}{1235}, \quad b_{4}^{\frac{15}{2}, \frac{7}{2}}=\frac{8}{182325} \nu\left(-565+\nu^{2}\right), \\
& b_{6}^{\frac{15}{2}, \frac{7}{2}}=-\frac{4}{920205}(-13+\nu)(-11+\nu) \nu(11+\nu)(13+\nu), b_{2}^{\frac{17}{2}, \frac{3}{2}}=-\frac{4 \nu}{255}, \\
& b_{2}^{\frac{17}{2}, \frac{5}{2}}=-\frac{4 \nu}{855}, \quad b_{4}^{\frac{17}{2}, \frac{5}{2}}=\frac{8}{49725}(-15+\nu) \nu(15+\nu), \quad b_{2}^{\frac{19}{2}, \frac{3}{2}}=-\frac{4 \nu}{323} .
\end{aligned}
$$

Some of the coefficients in eq. (C.15) overlap with the ones in (3.12). For example, the coefficient $b_{4}^{\frac{5}{2}, \frac{7}{2}}$ of eq. (C.15) is exactly the same as the coefficient $b_{6}^{4,4}$ of (3.12). The explicit $\nu$ dependence in the generalized hypergeometric functions can be determined similarly.

From eqs. (C.14) and (C.15), we obtain

$$
\begin{aligned}
a_{2 r}^{h_{1}+\frac{1}{2}, h_{2}+\frac{1}{2}}(\mu)= & { }_{4} F_{3}\left[\begin{array}{c}
\frac{1}{2}+\mu, \frac{1}{2}-\mu, \frac{2-2 r}{2}, \frac{1-2 r}{2} \\
\frac{1}{2}-h_{1}, \frac{1}{2}-h_{2}, \frac{3}{2}+h_{1}+h_{2}-2 r
\end{array} \mid 1\right] \\
& +{ }_{4} F_{3}\left[\begin{array}{c}
\frac{3}{2}-\mu,-\frac{1}{2}+\mu, \frac{2-2 r}{2}, \frac{1-2 r}{2} \\
\frac{1}{2}-h_{1}, \frac{1}{2}-h_{2}, \frac{3}{2}+h_{1}+h_{2}-2 r \mid 1
\end{array}\right], \\
b_{2 r}^{h_{1}+\frac{1}{2}, h_{2}+\frac{1}{2}}(\mu)= & { }_{4} F_{3}\left[\begin{array}{c}
\frac{1}{2}+\mu, \frac{1}{2}-\mu, \frac{1-2 r}{2}, \frac{-2 r}{2} \\
\frac{1}{2}-h_{1}, \frac{1}{2}-h_{2}, \frac{3}{2}+h_{1}+h_{2}-2 r
\end{array} \mid 1\right] \\
& -{ }_{4} F_{3}\left[\begin{array}{c}
\frac{3}{2}-\mu,-\frac{1}{2}+\mu, \frac{1-2 r}{2}, \frac{-2 r}{2} \\
\frac{1}{2}-h_{1}, \frac{1}{2}-h_{2}, \frac{3}{2}+h_{1}+h_{2}-2 r \mid
\end{array}\right] .
\end{aligned}
$$

It is easy to see that if we replace $2 r$ by $(2 r-1)$, then the above eq. (C.16) becomes the ones in eq. (C.12). This can be read from (4.8) and (4.9). 
D The (anti)commutators between the oscillators with nonzero $\mu$ in terms of generalized hypergeometric functions

- The case-one with two bosonic oscillators. We summarize the result (3.10) with (3.13) as follows:

$$
\begin{aligned}
& {[\underbrace{\hat{y}_{(1} \ldots \hat{y}_{1}}_{h_{1}-1+m} \underbrace{\left.\hat{y}_{2} \ldots \hat{y}_{2}\right)}_{h_{1}-1-m}, \underbrace{\hat{y}_{(1} \ldots \hat{y}_{1}}_{h_{2}-1+n} \underbrace{\left.\hat{y}_{2} \ldots \hat{y}_{2}\right)}_{h_{2}-1-n}]=} \\
& -\mathrm{i} \sum_{r=1}^{\left[\frac{h_{1}+h_{2}-1}{2}\right]}(-1)^{r} \frac{1}{(2 r-1) !} N_{2 r}^{h_{1}, h_{2}}(m, n)\left[\left({ }_{4} F_{3}\left[\begin{array}{c}
\frac{1}{2}+\mu, \frac{1}{2}-\mu, \frac{-2 r+1}{2}, \frac{-2 r+2}{2} \\
\frac{3}{2}-h_{1}, \frac{3}{2}-h_{2}, h_{1}+h_{2}-2 r+\frac{1}{2}
\end{array} \mid 1\right]\right.\right. \\
& \left.+{ }_{4} F_{3}\left[\begin{array}{c}
\frac{3}{2}-\mu,-\frac{1}{2}+\mu, \frac{-2 r+1}{2}, \frac{-2 r+2}{2} \\
\frac{3}{2}-h_{1}, \frac{3}{2}-h_{2}, h_{1}+h_{2}-2 r+\frac{1}{2}
\end{array} \mid 1\right]\right) \underbrace{\hat{y}_{(1} \ldots \ldots \ldots \hat{y}_{1}}_{h_{1}+h_{2}-2 r-1+m+n} \underbrace{\left.\hat{y}_{2} \ldots \ldots \ldots \ldots \hat{y}_{2}\right)}_{h_{1}+h_{2}-2 r-1-m-n} \\
& -\left({ }_{4} F_{3}\left[\begin{array}{c}
\frac{1}{2}+\mu, \frac{1}{2}-\mu, \frac{-2 r+1}{2}, \frac{-2 r+2}{2} \\
\frac{3}{2}-h_{1}, \frac{3}{2}-h_{2}, h_{1}+h_{2}-2 r+\frac{1}{2}
\end{array} \mid 1\right]\right. \\
& \left.-{ }_{4} F_{3}\left[\begin{array}{c|c}
\frac{3}{2}-\mu,-\frac{1}{2}+\mu, \frac{-2 r+1}{2}, \frac{-2 r+2}{2} & 1 \\
\frac{3}{2}-h_{1}, \frac{3}{2}-h_{2}, h_{1}+h_{2}-2 r+\frac{1}{2} & 1
\end{array}\right]\right) \\
& \times \underbrace{\hat{y}_{(1} \ldots \ldots \ldots \ldots \hat{y}_{1}}_{h_{1}+h_{2}-2 r-1+m+n} \underbrace{\hat{y}_{2} \ldots \ldots \ldots \ldots \hat{y}_{2)}}_{h_{1}+h_{2}-2 r-1-m-n} K] \text {, }
\end{aligned}
$$

which can be led to (4.5) and moreover reduces to eq. (B.1) for vanishing $\mu$.

- The case-two with one bosonic and one fermionic oscillators. From the result of eq. (C.4), we can present eq. (C.1) as follows:

$$
\begin{aligned}
& {[\underbrace{\hat{y}_{(1} \ldots \hat{y}_{1}}_{h_{1}-1+m} \underbrace{\hat{y}_{2} \ldots \hat{y}_{2}}_{h_{1}-1-m}, \underbrace{\hat{y}_{(1} \ldots \hat{y}_{1}}_{h_{2}-\frac{1}{2}+\rho} \underbrace{\hat{y}_{2} \ldots \hat{y}_{2)}}_{h_{2}-\frac{1}{2}-\rho}]=} \\
& \sum_{r=1}^{\left[\frac{h_{1}+h_{2}-1}{2}\right]}\left[\frac { - \mathrm { i } ( - 1 ) ^ { r } } { ( 2 r - 1 ) ! } N _ { 2 r } ^ { h _ { 1 } , h _ { 2 } + \frac { 1 } { 2 } } ( m , \rho ) \left({ }_{4} F_{3}\left[\begin{array}{c}
\frac{1}{2}+\mu, \frac{1}{2}-\mu, \frac{-2 r+1}{2}, \frac{-2 r+2}{2} \\
\frac{3}{2}-h_{1}, \frac{1}{2}-h_{2}, h_{1}+h_{2}-2 r+\frac{1}{2}
\end{array} \mid 1\right]\right.\right.
\end{aligned}
$$

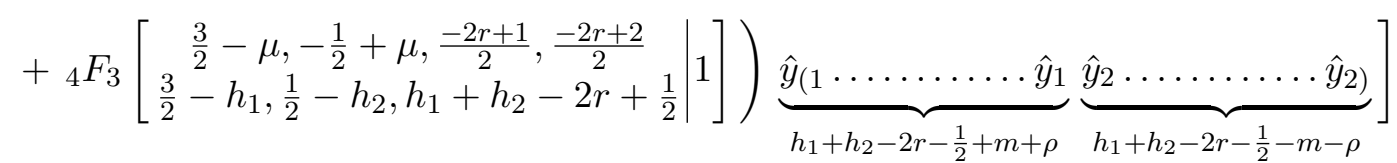

$$
\begin{aligned}
& +\sum_{r=1}^{\left[\frac{h_{1}+h_{2}}{2}\right]}\left[\frac { ( - 1 ) ^ { r } 2 ( h _ { 1 } - r ) } { ( 2 r - 1 ) ! } N _ { 2 r - 1 } ^ { h _ { 1 } , h _ { 2 } + \frac { 1 } { 2 } } ( m , \rho ) \left({ }_{4} F_{3}\left[\begin{array}{c}
\frac{1}{2}+\mu, \frac{1}{2}-\mu, \frac{-2 r+1}{2}, \frac{-2 r+2}{2} \\
\frac{3}{2}-h_{1}, \frac{1}{2}-h_{2}, h_{1}+h_{2}-2 r+\frac{3}{2}
\end{array} \mid 1\right]\right.\right. \\
& -{ }_{4} F_{3}\left[\begin{array}{c}
\frac{3}{2}-\mu,-\frac{1}{2}+\mu, \frac{-2 r+1}{2}, \frac{-2 r+2}{2} \\
\frac{3}{2}-h_{1}, \frac{1}{2}-h_{2}, h_{1}+h_{2}-2 r+\frac{3}{2} \mid
\end{array} \mid\right) \\
& \times \underbrace{\hat{y}_{(1} \ldots \ldots \ldots \ldots \hat{y}_{1}}_{h_{1}+h_{2}-2 r+\frac{1}{2}+m+\rho} \underbrace{\hat{y}_{2} \ldots \ldots \ldots \ldots \hat{y}_{2)}}_{h_{1}+h_{2}-2 r+\frac{1}{2}-m-\rho} K] \text {. }
\end{aligned}
$$

This can be rewritten as (4.6). 
In order to reproduce eq. (B.2) from eq. (D.2) by taking $\mu \rightarrow 0$, we consider the following expression

$$
\begin{aligned}
& \frac{1}{(2 r) !}\left(( 2 r + 1 ) { } _ { 4 } F _ { 3 } \left[\begin{array}{c|c}
\frac{1}{2}, \frac{1}{2}, \frac{-2 r}{2}, \frac{-2 r+1}{2} \\
\left.\frac{3}{2}-h_{1}, \frac{1}{2}-h_{2}, h_{1}+h_{2}-2 r+\frac{1}{2} \mid 1\right]
\end{array}\right.\right. \\
& \left.+(2 r-1){ }_{4} F_{3}\left[\begin{array}{c}
\frac{3}{2},-\frac{1}{2}, \frac{-2 r}{2}, \frac{-2 r+1}{2} \\
\frac{3}{2}-h_{1}, \frac{1}{2}-h_{2}, h_{1}+h_{2}-2 r+\frac{1}{2} \mid
\end{array} \mid 1\right]\right),
\end{aligned}
$$

which contains

$$
\frac{(2 r+1)}{(2 r) !}\left(\frac{1}{2}\right)_{n}\left(\frac{1}{2}\right)_{n}(-r)_{n}\left(\frac{-2 r+1}{2}\right)_{n}+\frac{(2 r-1)}{(2 r) !}\left(\frac{3}{2}\right)_{n}\left(\frac{-1}{2}\right)_{n}(-r)_{n}\left(\frac{-2 r+1}{2}\right)_{n} .
$$

Here we take $(n+1)$-th term of generalized hypergeometric function. On the other hand, the following combination

$$
\begin{aligned}
\frac{1}{(2 r-1) !}\left({ } _ { 4 } F _ { 3 } \left[\begin{array}{c}
\frac{1}{2}, \frac{1}{2}, \frac{-2 r+1}{2}, \frac{-2 r+2}{2} \\
\frac{3}{2}-h_{1}, \frac{1}{2}-h_{2}, h_{1}+h_{2}-2 r+\frac{1}{2} \mid
\end{array} \mid\right.\right. & 1] \\
& \left.+{ }_{4} F_{3}\left[\begin{array}{c}
\frac{3}{2},-\frac{1}{2}, \frac{-2 r+1}{2}, \frac{-2 r+2}{2} \\
\frac{3}{2}-h_{1}, \frac{1}{2}-h_{2}, h_{1}+h_{2}-2 r+\frac{1}{2}
\end{array} \mid 1\right]\right)
\end{aligned}
$$

contains

$$
\frac{1}{(2 r-1) !}\left(\frac{1}{2}\right)_{n}\left(\frac{1}{2}\right)_{n}\left(\frac{-2 r+1}{2}\right)_{n}(1-r)_{n}+\frac{1}{(2 r-1) !}\left(\frac{3}{2}\right)_{n}\left(\frac{-1}{2}\right)_{n}\left(\frac{-2 r+1}{2}\right)_{n}(1-r)_{n}
$$

in the $(n+1)$-th term. Note that the lower three elements in the generalized hypergeometric functions in eq. (D.3) and eq. (D.5) are common.

Then the relations eq. (D.4) and eq. (D.6) are equivalent to each other

$$
\begin{aligned}
& \left((2 r+1)\left(\frac{1}{2}\right)_{n}\left(\frac{1}{2}\right)_{n}+(2 r-1)\left(\frac{-1}{2}\right)_{n}\left(\frac{3}{2}\right)_{n}\right)(-r)_{n} \\
= & 2 r\left(\left(\frac{1}{2}\right)_{n}\left(\frac{1}{2}\right)_{n}+\left(\frac{-1}{2}\right)_{n}\left(\frac{3}{2}\right)_{n}\right)(1-r)_{n} .
\end{aligned}
$$

It is easy to check this identity eq. (D.7) from

$$
\begin{aligned}
\left(\frac{1}{2}\right)_{n} & =-2\left(n-\frac{1}{2}\right)\left(\frac{-1}{2}\right)_{n}, & \left(\frac{3}{2}\right)_{n} & =2\left(n+\frac{1}{2}\right)\left(\frac{1}{2}\right)_{n}, \\
\left(\frac{1}{2}\right)_{n}\left(\frac{1}{2}\right)_{n} & =-\frac{n-\frac{1}{2}}{n+\frac{1}{2}}\left(\frac{-1}{2}\right)_{n}\left(\frac{3}{2}\right)_{n}, & \frac{(1-r)_{n}}{(-r)_{n}} & =1-\frac{n}{r} .
\end{aligned}
$$

Therefore, eq. (B.2) can be obtained from eq. (D.2) if we take $\mu \rightarrow 0$. 
Similarly, although we have not presented here for the complete expressions, the next one can be written as

$$
\begin{aligned}
& {[\underbrace{\hat{y}_{\left(1 \ldots \hat{y}_{1}\right.}}_{h_{1}-1+m} \underbrace{\hat{y}_{2} \ldots \hat{y}_{2)}}_{h_{1}-1-m} K, \underbrace{\hat{y}_{(1} \ldots \hat{y}_{1}}_{h_{2}-\frac{1}{2}+\rho} \underbrace{\left.\hat{y}_{2} \ldots \hat{y}_{2}\right)}_{h_{2}-\frac{1}{2}-\rho}]=} \\
& \sum_{r=1}^{\left[\frac{h_{1}+h_{2}}{2}\right]}\left[\frac { ( - 1 ) ^ { r } } { ( 2 r - 2 ) ! } N _ { 2 r - 1 } ^ { h _ { 1 } , h _ { 2 } + \frac { 1 } { 2 } } ( m , \rho ) \left({ }_{4} F_{3}\left[\begin{array}{c}
\frac{1}{2}+\mu, \frac{1}{2}-\mu, \frac{-2 r+2}{2}, \frac{-2 r+3}{2} \\
\frac{3}{2}-h_{1}, \frac{1}{2}-h_{2}, h_{1}+h_{2}-2 r+\frac{3}{2}
\end{array} \mid 1\right]\right.\right. \\
& \left.+{ }_{4} F_{3}\left[\begin{array}{c|c}
\frac{3}{2}-\mu,-\frac{1}{2}+\mu, \frac{-2 r+2}{2}, \frac{-2 r+3}{2} \\
\frac{3}{2}-h_{1}, \frac{1}{2}-h_{2}, h_{1}+h_{2}-2 r+\frac{3}{2}
\end{array} \mid 1\right]\right) \\
& \times \underbrace{\hat{y}_{(1} \ldots \ldots \ldots \ldots \hat{y}_{1}}_{h_{1}+h_{2}-2 r+\frac{1}{2}+m+\rho} \underbrace{\left.\hat{y}_{2} \ldots \ldots \ldots \ldots \hat{y}_{2}\right)}_{h_{1}+h_{2}-2 r+\frac{1}{2}-m-\rho} K] \\
& -\mathrm{i} \sum_{r=1}^{\left[\frac{h_{1}+h_{2}-1}{2}\right]}\left[\frac { ( - 1 ) ^ { r } ( h _ { 1 } - r - \frac { 1 } { 2 } ) } { r ( 2 r - 1 ) ! } N _ { 2 r } ^ { h _ { 1 } , h _ { 2 } + \frac { 1 } { 2 } } ( m , \rho ) \left({ }_{4} F_{3}\left[\begin{array}{c}
\frac{1}{2}+\mu, \frac{1}{2}-\mu, \frac{-2 r}{2}, \frac{-2 r+1}{2} \\
\frac{3}{2}-h_{1}, \frac{1}{2}-h_{2}, h_{1}+h_{2}-2 r+\frac{1}{2}
\end{array} \mid 1\right]\right.\right. \\
& \left.-{ }_{4} F_{3}\left[\begin{array}{c}
\frac{3}{2}-\mu,-\frac{1}{2}+\mu, \frac{-2 r}{2}, \frac{-2 r+1}{2} \\
\frac{3}{2}-h_{1}, \frac{1}{2}-h_{2}, h_{1}+h_{2}-2 r+\frac{1}{2}
\end{array} \mid 1\right]\right) \underbrace{\hat{y}_{(1} \ldots \ldots \ldots \ldots \hat{y}_{1}}_{h_{1}+h_{2}-2 r-\frac{1}{2}+m+\rho} \underbrace{\left.\hat{y}_{2} \ldots \ldots \ldots \ldots \hat{y}_{2}\right)}_{h_{1}+h_{2}-2 r-\frac{1}{2}-m-\rho}] \text {, }
\end{aligned}
$$

which can be rewritten as (4.7) and this leads to eq. (B.3) when we take $\mu \rightarrow 0$.

- The case-three with two fermionic oscillators. By combining the previous result eq. (C.9) with eq. (C.12), we obtain

$$
\begin{aligned}
& \{\underbrace{\hat{y}_{(1} \ldots \hat{y}_{1}}_{h_{1}-\frac{1}{2}+\rho} \underbrace{\left.\hat{y}_{2} \ldots \hat{y}_{2}\right)}_{h_{1}-\frac{1}{2}-\rho}, \underbrace{\hat{y}_{(1} \ldots \hat{y}_{1}}_{h_{2}-\frac{1}{2}+\omega} \underbrace{\left.\hat{y}_{2} \ldots \hat{y}_{2}\right)}_{h_{2}-\frac{1}{2}-\omega}\} \\
& =\sum_{r=1}^{\left[\frac{h_{1}+h_{2}+1}{2}\right]}(-1)^{r} N_{2 r-1}^{h_{1}+\frac{1}{2}, h_{2}+\frac{1}{2}}(\rho, \omega)\left[-\frac{1}{(2 r-2) !}\right. \\
& \times\left({ }_{4} F_{3}\left[\begin{array}{c|c}
\frac{1}{2}+\mu, \frac{1}{2}-\mu, \frac{-2 r+2}{2}, \frac{-2 r+3}{2} \\
\frac{1}{2}-h_{1}, \frac{1}{2}-h_{2}, h_{1}+h_{2}-2 r+\frac{5}{2}
\end{array} \mid 1\right]\right. \\
& \left.+{ }_{4} F_{3}\left[\begin{array}{c}
\frac{3}{2}-\mu,-\frac{1}{2}+\mu, \frac{-2 r+2}{2}, \frac{-2 r+3}{2} \\
\frac{1}{2}-h_{1}, \frac{1}{2}-h_{2}, h_{1}+h_{2}-2 r+\frac{5}{2}
\end{array} \mid 1\right]\right) \underbrace{\hat{y}_{\left(1 \ldots \ldots . . \hat{y}_{1}\right.}}_{h_{1}+h_{2}-2 r+1+\rho+\omega} \underbrace{\hat{y}_{2} \ldots \ldots \ldots \hat{y}_{2)}}_{h_{1}+h_{2}-2 r+1-\rho-\omega} \\
& +\frac{2\left(h_{1}+h_{2}-r+1\right)}{(2 r-1) !}\left({ }_{4} F_{3}\left[\begin{array}{c|c}
\frac{1}{2}+\mu, \frac{1}{2}-\mu, \frac{-2 r+1}{2}, \frac{-2 r+2}{2} \\
\frac{1}{2}-h_{1}, \frac{1}{2}-h_{2}, h_{1}+h_{2}-2 r+\frac{5}{2}
\end{array} \mid 1\right]\right. \\
& \left.-{ }_{4} F_{3}\left[\begin{array}{c|c}
\frac{3}{2}-\mu,-\frac{1}{2}+\mu, \frac{-2 r+1}{2}, \frac{-2 r+2}{2} \\
\frac{1}{2}-h_{1}, \frac{1}{2}-h_{2}, h_{1}+h_{2}-2 r+\frac{5}{2}
\end{array} \mid 1\right]\right) \\
& \times \underbrace{\hat{y}_{(1} \ldots \ldots \ldots \hat{y}_{1}}_{h_{1}+h_{2}-2 r+1+\rho+\omega} \underbrace{\left.\hat{y}_{2} \ldots \ldots \ldots \hat{y}_{2}\right)}_{h_{1}+h_{2}-2 r+1-\rho-\omega} K] \text {, }
\end{aligned}
$$

which can be expressed as (4.8). By taking $\mu \rightarrow 0$, we obtain the previous eq. (B.4). 
There is a relation

$$
\begin{aligned}
& { }_{4} F_{3}\left[\begin{array}{c|c}
\frac{1}{2},-\frac{1}{2}, \frac{-2 r+2}{2}, \frac{-2 r+3}{2} \\
\frac{1}{2}-h_{1}, \frac{1}{2}-h_{2}, h_{1}+h_{2}-2 r+\frac{5}{2}
\end{array} \mid 1\right] \\
& =\frac{1}{2}\left({ } _ { 4 } F _ { 3 } \left[\begin{array}{c}
\frac{1}{2}, \frac{1}{2}, \frac{-2 r+2}{2}, \frac{-2 r+3}{2} \\
\frac{1}{2}-h_{1}, \frac{1}{2}-h_{2}, h_{1}+h_{2}-2 r+\frac{5}{2} \mid
\end{array}|1|\right.\right. \\
& \left.\quad+{ }_{4} F_{3}\left[\begin{array}{c}
\frac{3}{2},-\frac{1}{2}, \frac{-2 r+2}{2}, \frac{-2 r+3}{2} \\
\frac{1}{2}-h_{1}, \frac{1}{2}-h_{2}, h_{1}+h_{2}-2 r+\frac{5}{2}
\end{array} \mid 1\right]\right) .
\end{aligned}
$$

This eq. (D.11) can be checked, similar to eq. (D.8), by realizing that

$$
\begin{aligned}
2\left(\frac{-1}{2}\right)_{n}\left(\frac{1}{2}\right)_{n} & =\left(\frac{1}{2}\right)_{n}\left(\frac{1}{2}\right)_{n}+\left(\frac{-1}{2}\right)_{n}\left(\frac{3}{2}\right)_{n} \\
& =-2\left(n-\frac{1}{2}\right)\left(\frac{-1}{2}\right)_{n}\left(\frac{1}{2}\right)_{n}+2\left(n+\frac{1}{2}\right)\left(\frac{-1}{2}\right)_{n}\left(\frac{1}{2}\right)_{n} .
\end{aligned}
$$

Then we observe that when we take $\mu \rightarrow 0$, the relation eq. (D.10) becomes the relation eq. (B.4) with eq. (D.12).

Finally, we obtain

$$
\begin{aligned}
& \{\underbrace{\hat{y}_{(1} \ldots \hat{y}_{1}}_{h_{1}-\frac{1}{2}+\rho} \underbrace{\left.\hat{y}_{2} \ldots \hat{y}_{2}\right)}_{h_{1}-\frac{1}{2}-\rho}, \underbrace{\hat{y}_{(1} \ldots \hat{y}_{1}}_{h_{2}-\frac{1}{2}+\omega} \underbrace{\hat{y}_{2} \ldots \hat{y}_{2)}}_{h_{2}-\frac{1}{2}-\omega} K\} \\
& =\mathrm{i} \sum_{r=1}^{\left[\frac{h_{1}+h_{2}}{2}\right]}(-1)^{r} N_{2 r}^{h_{1}+\frac{1}{2}, h_{2}+\frac{1}{2}}(\rho, \omega)\left[-\frac{1}{(2 r-1) !}\right. \\
& \times\left({ }_{4} F_{3}\left[\begin{array}{c|c}
\frac{1}{2}+\mu, \frac{1}{2}-\mu, \frac{-2 r+1}{2}, \frac{-2 r+2}{2} \\
\frac{1}{2}-h_{1}, \frac{1}{2}-h_{2}, h_{1}+h_{2}-2 r+\frac{3}{2}
\end{array} \mid 1\right]\right.
\end{aligned}
$$

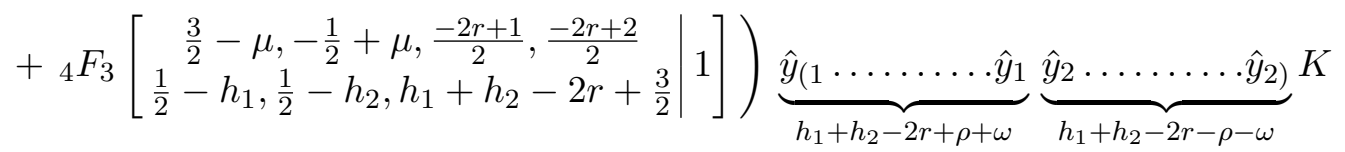

$$
\begin{aligned}
& +\frac{2\left(h_{1}+h_{2}-r\right)+1}{(2 r) !}\left({ }_{4} F_{3}\left[\begin{array}{c}
\frac{1}{2}+\mu, \frac{1}{2}-\mu, \frac{-2 r}{2}, \frac{-2 r+1}{2} \\
\frac{1}{2}-h_{1}, \frac{1}{2}-h_{2}, h_{1}+h_{2}-2 r+\frac{3}{2}
\end{array} \mid 1\right]\right. \\
& \left.-{ }_{4} F_{3}\left[\begin{array}{c|c}
\frac{3}{2}-\mu,-\frac{1}{2}+\mu, \frac{-2 r}{2}, \frac{-2 r+1}{2} \\
\frac{1}{2}-h_{1}, \frac{1}{2}-h_{2}, h_{1}+h_{2}-2 r+\frac{3}{2}
\end{array} \mid 1\right]\right) \underbrace{\hat{y}_{(1} \ldots \ldots \ldots \hat{y}_{1}}_{h_{1}+h_{2}-2 r+\rho+\omega} \underbrace{\left.\hat{y}_{2} \ldots \ldots \ldots \hat{y}_{2}\right)}_{h_{1}+h_{2}-2 r-\rho-\omega}] \text {, }
\end{aligned}
$$

which is equal to (4.9) by using the relations (4.2). When we take $\mu \rightarrow 0$, the relation eq. (D.13) becomes the relation eq. (B.5). 


\section{E The remaining (anti)commutators between the $\mathcal{N}=4$ higher spin generators}

By following the procedure in section 4, the remaining ten (anti)commutators are described as follows. ${ }^{22}$

\section{E.1 The four (anti)commutators with common $\Phi_{\frac{1}{2}, \rho}^{\left(s_{1}\right), i}$}

The four kinds of (anti)commutators are given in this subsection. By using the relations (4.8) and (4.9), we obtain

$$
\begin{aligned}
\left\{\Phi_{\frac{1}{2}, \rho}^{\left(s_{1}\right), i}, \Phi_{\frac{1}{2}, \omega}^{\left(s_{2}\right), j}\right\}= & -\delta^{i j} \sum_{r=1}^{\left[\frac{s_{1}+s_{2}}{2}\right]} \mathrm{i}(-1)^{r}\left(2 s_{1}-1\right)\left(2 s_{2}-1\right)[ \\
& \frac{2\left(s_{1}+s_{2}-r+1\right)}{(2 r-1)\left(2 s_{1}+2 s_{2}-4 r+3\right)} \mathrm{FF}_{2 r-1,-}^{s_{1}+\frac{1}{2}, s_{2}+\frac{1}{2}}(\rho, \omega ; \mu) \Phi_{0, \rho+\omega}^{\left(s_{1}+s_{2}-2 r+2\right)} \\
& +\left(\frac{1}{2\left(s_{1}+s_{2}-2 r\right)-1} \mathrm{FF}_{2 r-1,+}^{s_{1}+\frac{1}{2}, s_{2}+\frac{1}{2}}(\rho, \omega ; \mu)\right. \\
& \left.\left.+\frac{2 \nu\left(s_{1}+s_{2}+1-r\right)}{(2 r-1)\left(2 s_{1}+2 s_{2}-4 r+3\right)} \mathrm{FF}_{2 r-1,-}^{s_{1}+\frac{1}{2}, s_{2}+\frac{1}{2}}(\rho, \omega ; \mu)\right) \tilde{\Phi}_{2, \rho+\omega}^{\left(s_{1}+s_{2}-2 r\right)}\right] \\
& -\left[\frac{\left.\sum_{r=1}^{2}\right]}{\mathrm{i}(-1)^{r} \frac{\left(2 s_{1}-1\right)\left(2 s_{2}-1\right)}{2\left(s_{1}+s_{2}-2 r\right)-1}\left[\mathrm{FF}_{2 r,+}^{s_{1}+\frac{1}{2}, s_{2}+\frac{1}{2}}(\rho, \omega ; \mu) \Phi_{1, \rho+\omega}^{\left(s_{1}+s_{2}-2 r\right), i j}\right.}\right. \\
& \left.+\frac{2\left(s_{1}+s_{2}-r\right)+1}{2 r} \mathrm{FF}_{2 r,-}^{s_{1}+\frac{1}{2}, s_{2}+\frac{1}{2}}(\rho, \omega ; \mu) \Phi_{1, \rho+\omega}^{\left(s_{1}+s_{2}-2 r\right), i j}\right] .
\end{aligned}
$$

It is obvious that for the indices $i=j$, the square of Pauli matrix is proportional to the $2 \times 2$ identity matrix and this leads to the above (bosonic) $\mathcal{N}=4$ higher spin generators having $\delta^{i j}$ tensor. For the indices $i \neq j$, the similar analysis to (4.15) provides the above $\mathcal{N}=4$ higher spin generators with $\mathrm{SO}(4)$ adjoint indices. There is a linear $\nu$ dependence in the coefficient.

\footnotetext{
${ }^{22}$ For convenience, we present some values of structure constants here. The structure constants $\mathrm{BB}_{r,+}^{h_{1}, h_{2}}$ contain $\left(\nu^{2}-15329555\right),\left(\nu^{8}-18824252 \nu^{6}+80986952555566 \nu^{4}-112329283433337991596 \nu^{2}+\right.$ $46136714396921922707472825)$ and $(-197+\nu)(-195+\nu) \cdots(-3+\nu)(3+\nu)(5+\nu) \cdots(197+\nu)\left(-199+\nu^{2}\right)$ for $r=3,10$ and $r=199$ together with $h_{1}=h_{2}=100$ respectively. The structure constants $\mathrm{BB}_{r,-}^{h_{1}, h_{2}}$ contain $\nu, \nu\left(\nu^{6}-14118203 \nu^{4}+40493570399155 \nu^{2}-28082401845512944761\right)$, and $(-197+\nu)(-195+\nu) \cdots(-3+$ $\nu) \nu(3+\nu)(5+\nu) \cdots(197+\nu)$ for above values. The structure constants $\mathrm{BF}_{r,+}^{h_{1}, h_{2}+\frac{1}{2}}$ contain $\left(15368364-\nu^{2}\right)$, $\left(5 \nu^{8}-94369560 \nu^{6}+407046639208272 \nu^{4}-565989214693985201408 \nu^{2}+233034068993325158419660800\right)$ and $(-198+\nu)(-196+\nu) \cdots(-2+\nu)(2+\nu)(4+\nu) \cdots(198+\nu)$ for above values of $r, h_{1}, h_{2}$. The structure constants $\mathrm{BF}_{r,-}^{h_{1}, h_{2}+\frac{1}{2}}$ contain $\nu, \nu\left(\nu^{8}-55732440 \nu^{6}+394459539349008 \nu^{4}-756192834594836501248 \nu^{2}+\right.$ $394300540856824919467204608)$ and $(-198+\nu)(-196+\nu) \cdots(-4+\nu) \nu(4+\nu)(6+\nu) \cdots(198+\nu)$ for $r=3,10$ and $r=198$ with $h_{1}=h_{2}=100$ respectively. Finally, the structure constants $\mathrm{FF}_{r,+}^{h_{1}+\frac{1}{2}, h_{2}+\frac{1}{2}}$ contain $\left(15485580-\nu^{2}\right)$ and $\left(\nu^{8}-19021880 \nu^{6}+82687450448656 \nu^{4}-115867186219647371520 \nu^{2}+\right.$ 48074201136264436025131008) for $r=3$ and 10 with $h_{1}=h_{2}=100$ respectively. The structure constants $\mathrm{FF}_{r,-}^{h_{1}+\frac{1}{2}, h_{2}+\frac{1}{2}}$ contain $\nu$ and $\nu\left(\nu^{8}-56171720 \nu^{6}+400685503988368 \nu^{4}-774118850666523886080 \nu^{2}+\right.$ 406779762639433803784851456) for above values. In this case there is no simple factorized form compared to other cases. The even and odd powers of $\nu$ are observed alternatively.
} 
With (4.6) and (4.7), we determine the following commutator

$$
\begin{aligned}
& {\left[\Phi_{\frac{1}{2}, \rho}^{\left(s_{1}\right), i}, \Phi_{1, m}^{\left(s_{2}\right), j k}\right]=\left(-\delta^{i j} \mathrm{i}\left(2 s_{1}-1\right)\left(2 s_{2}-1\right)[\right.} \\
& \sum_{r=1}^{\left[\frac{s_{1}+s_{2}}{2}\right]}(-1)^{r} \frac{2\left(s_{2}-r\right)+1}{2 r\left(2 s_{1}+2 s_{2}-4 r-1\right)} \mathrm{BF}_{2 r,-}^{s_{2}+1, s_{1}+\frac{1}{2}}(m, \rho ; \mu) \tilde{\Phi}_{\frac{3}{2}, \rho+m}^{\left(s_{1}+s_{2}-2 r\right), k} \\
& \left.\left.+\sum_{r=1}^{\left[\frac{s_{1}+s_{2}+1}{2}\right]}(-1)^{r} \frac{1}{2\left(s_{1}+s_{2}-2 r\right)+3} \mathrm{BF}_{2 r-1,+}^{s_{2}+1, s_{1}+\frac{1}{2}}(m, \rho ; \mu) \Phi_{\frac{1}{2}, \rho+m}^{\left(s_{1}+s_{2}-2 r+2\right), k}\right]-(j \longleftrightarrow k)\right) \\
& +\epsilon^{i j k l} \mathrm{i}\left(2 s_{1}-1\right)\left(2 s_{2}-1\right)\left[\sum_{r=1}^{\left[\frac{s_{1}+s_{2}}{2}\right]}(-1)^{r} \frac{1}{2\left(s_{1}+s_{2}-2 r\right)-1} \mathrm{BF}_{2 r,+}^{s_{2}+1, s_{1}+\frac{1}{2}}(m, \rho ; \mu) \tilde{\Phi}_{\frac{3}{2}, \rho+m}^{\left(s_{1}+s_{2}-2 r\right), l}\right. \\
& \left.+\sum_{r=1}^{\left[\frac{s_{1}+s_{2}+1}{2}\right]}(-1)^{r} \frac{2\left(s_{2}-r+1\right)}{(2 r-1)\left(2 s_{1}+2 s_{2}-4 r+3\right)} \mathrm{BF}_{2 r-1,-}^{s_{2}+1, s_{1}+\frac{1}{2}}(m, \rho ; \mu) \Phi_{\frac{1}{2}, \rho+m}^{\left(s_{1}+s_{2}+2-2 r\right), l}\right] .
\end{aligned}
$$

We should use the simple relations between the Pauli matrices in order to deal with the $2 \times 2$ matrices in the tensor product.

We obtain the anticommutator with (4.8) and (4.9)

$$
\begin{aligned}
& \left\{\Phi_{\frac{1}{2}, \rho}^{\left(s_{1}\right), i}, \Phi_{\frac{3}{2}, \omega}^{\left(s_{2}\right), j}\right\}=\delta^{i j} \mathrm{i}\left(2 s_{1}-1\right)\left(2 s_{2}-1\right)[ \\
& \sum_{r=1}^{\left[\frac{s_{1}+s_{2}+1}{2}\right]}(-1)^{r} \frac{1}{2\left(s_{1}+s_{2}-2 r\right)+3} \mathrm{FF}_{2 r,+}^{s_{1}+\frac{1}{2}, s_{2}+\frac{3}{2}}(\rho, \omega ; \mu) \Phi_{0, \rho+\omega}^{\left(s_{1}+s_{2}-2 r+2\right)} \\
& +\sum_{r=1}^{\left[\frac{s_{1}+s_{2}}{2}\right]}(-1)^{r} \frac{1}{2\left(s_{1}+s_{2}-2 r\right)-1}\left(\frac{\nu}{\left(2 s_{1}+2 s_{2}-4 r+3\right)} \mathrm{FF}_{2 r,+}^{s_{1}+\frac{1}{2}, s_{2}+\frac{3}{2}}(\rho, \omega ; \mu)\right. \\
& \left.\left.+\frac{2\left(s_{1}+s_{2}-r\right)+3}{2 r} \mathrm{FF}_{2 r,-}^{s_{1}+\frac{1}{2}, s_{2}+\frac{3}{2}}(\rho, \omega ; \mu)\right) \tilde{\Phi}_{2, \rho+\omega}^{\left(s_{1}+s_{2}-2 r\right)}\right] \\
& -\sum_{r=1}^{\left[\frac{s_{1}+s_{2}+1}{2}\right]} \frac{\mathrm{i}(-1)^{r}\left(2 s_{1}-1\right)\left(2 s_{2}-1\right)}{2\left(s_{1}+s_{2}-2 r\right)+3}[ \\
& \frac{2\left(s_{1}+s_{2}-r\right)+4}{(2 r-1)} \mathrm{FF}_{2 r-1,-}^{s_{1}+\frac{1}{2}, s_{2}+\frac{3}{2}}(\rho, \omega ; \mu) \Phi_{1, \rho+\omega}^{\left(s_{1}+s_{2}-2 r+2\right), i j} \\
& \left.+\mathrm{FF}_{2 r-1,+}^{s_{1}+\frac{1}{2}, s_{2}+\frac{3}{2}}(\rho, \omega ; \mu) \Phi_{1, \rho+\omega}^{\left(s_{1}+s_{2}-2 r+2\right), i j}\right] .
\end{aligned}
$$

Again the linear $\nu$ dependence appears in the coefficient. 
By applying the relation (4.6), the following commutator can be determined

$$
\begin{aligned}
& {\left[\Phi_{\frac{1}{2}, \rho}^{\left(s_{1}, i\right.}, \tilde{\Phi}_{2, m}^{\left(s_{2}\right)}\right]=\mathrm{i}\left(2 s_{1}-1\right)\left(2 s_{2}-1\right)[Q} \\
& {\left[\frac{s_{1}+s_{2}+1}{2}\right]} \\
& \sum_{r=1}^{2}(-1)^{r} \frac{1}{2\left(s_{1}+s_{2}-2 r\right)+3} \mathrm{BF}_{2 r,+}^{s_{2}+2, s_{1}+\frac{1}{2}}(m, \rho ; \mu) \Phi_{\frac{1}{2}, \rho+m}^{\left(s_{1}+s_{2}-2 r+2\right), i} \\
& \left.-\sum_{r=1}^{\left[\frac{s_{1}+s_{2}}{2}\right]}(-1)^{r} \frac{1}{2\left(s_{1}+s_{2}-2 r\right)+3} \frac{2\left(s_{2}-r+2\right)}{(2 r-1)} \mathrm{BF}_{2 r-1,-}^{s_{2}+2, s_{1}+\frac{1}{2}}(m, \rho ; \mu) \tilde{\Phi}_{\frac{3}{2}, \rho+m}^{\left(s_{1}+s_{2}-2 r+2\right), i}\right] .
\end{aligned}
$$

\section{E.2 The three (anti)commutators with common $\Phi_{1, m}^{\left(s_{1}\right), i j}$}

The three kinds of commutators are given in this subsection. By using (4.5) and (4.14), we obtain the commutator

$$
\begin{aligned}
& {\left[\Phi_{1, m}^{\left(s_{1}\right), i j}, \Phi_{1, n}^{\left(s_{2}\right), k l}\right]=-\left(\delta^{i k} \delta^{j l}-\delta^{i l} \delta^{j k}\right) \mathrm{i}\left(2 s_{1}-1\right)\left(2 s_{2}-1\right)[} \\
& \sum_{r=1}^{\left[\frac{s_{1}+s_{2}+1}{2}\right]}(-1)^{r} \frac{1}{2\left(s_{1}+s_{2}-2 r\right)+3} \mathrm{BB}_{2 r,-}^{s_{1}+1, s_{2}+1}(m, n ; \mu) \Phi_{0, m+n}^{\left(s_{1}+s_{2}-2 r+2\right)} \\
& +\sum_{r=1}^{\left[\frac{s_{1}+s_{2}}{2}\right]}(-1)^{r} \frac{1}{2\left(s_{1}+s_{2}-2 r\right)-1} \\
& \left.\times\left(\mathrm{BB}_{2 r,+}^{s_{1}+1, s_{2}+1}(m, n ; \mu)+\frac{\nu}{2\left(s_{1}+s_{2}-2 r\right)+3} \mathrm{BB}_{2 r,-}^{s_{1}+1, s_{2}+1}(m, n ; \mu)\right) \tilde{\Phi}_{2, m+n}^{\left(s_{1}+s_{2}-2 r\right)}\right] \\
& -\epsilon^{i j k l} \mathrm{i}\left(2 s_{1}-1\right)\left(2 s_{2}-1\right)[ \\
& \sum_{r=1}^{\left[\frac{s_{1}+s_{2}+1}{2}\right]}(-1)^{r} \frac{1}{2\left(s_{1}+s_{2}-2 r\right)+3} \mathrm{BB}_{2 r,+}^{s_{1}+1, s_{2}+1}(m, n ; \mu) \Phi_{0, m+n}^{\left(s_{1}+s_{2}-2 r+2\right)} \\
& +\sum_{r=1}^{\left[\frac{s_{1}+s_{2}}{2}\right]}(-1)^{r} \frac{1}{2\left(s_{1}+s_{2}-2 r\right)-1} \\
& \left.\times\left(\frac{\nu}{2\left(s_{1}+s_{2}-2 r\right)+3} \mathrm{BB}_{2 r,+}^{s_{1}+1, s_{2}+1}(m, n ; \mu)+\mathrm{BB}_{2 r,-}^{s_{1}+1, s_{2}+1}(m, n ; \mu)\right) \tilde{\Phi}_{2, m+n}^{\left(s_{1}+s_{2}-2 r\right)}\right] \\
& +\left(\delta ^ { i k } \sum _ { r = 1 } ^ { [ \frac { s _ { 1 } + s _ { 2 } + 2 } { 2 } ] } \frac { \mathrm { i } ( - 1 ) ^ { r } ( 2 s _ { 1 } - 1 ) ( 2 s _ { 2 } - 1 ) } { 2 ( s _ { 1 } + s _ { 2 } - 2 r ) + 3 } \left[\mathrm{BB}_{2 r-1,+}^{s_{1}+1, s_{2}+1}(m, n ; \mu) \Phi_{1, m+n}^{\left(s_{1}+s_{2}-2 r+2\right), j l}\right.\right. \\
& \left.\left.+\mathrm{BB}_{2 r-1,-}^{s_{1}+1, s_{2}+1}(m, n ; \mu) \Phi_{1, m+n}^{\left(s_{1}+s_{2}-2 r+2\right), j l}\right]-(k \longleftrightarrow l)-(i \longleftrightarrow j)+(i \longleftrightarrow j, k \longleftrightarrow l)\right)
\end{aligned}
$$

The linear $\nu$ dependence appears in the coefficient coming from the replacement of the $\mathcal{N}=4$ higher spin generators in (2.6). 
By using (4.6) and (4.7), we determine the following commutator

$$
\begin{aligned}
& {\left[\Phi_{1, m}^{\left(s_{1}\right), i j}, \tilde{\Phi}_{\frac{3}{2}, \rho}^{\left(s_{2}\right), k}\right]=\left(-\delta^{i k} \mathrm{i}\left(2 s_{1}-1\right)\left(2 s_{2}-1\right)[\right.} \\
& {\left[\frac{1}{\left.\sum_{r=1}^{s_{1}+s_{2}+1}\right]}(-1)^{r} \frac{1}{2\left(s_{1}+s_{2}-2 r\right)+3} \frac{2\left(s_{1}-r\right)+1}{2 r} \mathrm{BF}_{2 r,-}^{s_{1}+1, s_{2}+\frac{3}{2}}(m, \rho ; \mu) \Phi_{\frac{1}{2}, m+\rho}^{\left(s_{1}+s_{2}-2 r+2\right), j}\right.} \\
& \left.\left.-\sum_{r=1}^{\left[\frac{s_{1}+s_{2}+2}{2}\right]}(-1)^{r} \frac{1}{2\left(s_{1}+s_{2}-2 r\right)+3} \mathrm{BF}_{2 r-1,+}^{s_{1}+1, s_{2}+\frac{3}{2}}(m, \rho ; \mu) \tilde{\Phi}_{\frac{3}{2}, m+\rho}^{\left(s_{1}+s_{2}-2 r+2\right), j}\right]-(i \longleftrightarrow j)\right) \\
& +\epsilon^{i j k l} \mathrm{i}\left(2 s_{1}-1\right)\left(2 s_{2}-1\right)[ \\
& {\left[\frac{s_{1}+s_{2}+1}{2}\right]} \\
& \sum_{r=1}^{2}(-1)^{r} \frac{1}{2\left(s_{1}+s_{2}-2 r\right)+3} \mathrm{BF}_{2 r,+}^{s_{1}+1, s_{2}+\frac{3}{2}}(m, \rho ; \mu) \Phi_{\frac{1}{2}, m+\rho}^{\left(s_{1}+s_{2}-2 r+2\right), l} \\
& \left.-\sum_{r=1}^{\left[\frac{s_{1}+s_{2}+2}{2}\right]}(-1)^{r} \frac{1}{2\left(s_{1}+s_{2}-2 r\right)+3} \frac{2\left(s_{1}-r+1\right)}{(2 r-1)} \mathrm{BF}_{2 r-1,-}^{s_{1}+1, s_{2}+\frac{3}{2}}(m, \rho ; \mu) \tilde{\Phi}_{\frac{3}{2}, m+\rho}^{\left(s_{1}+s_{2}-2 r+2\right), l}\right] .
\end{aligned}
$$

From (4.5), we obtain

$$
\begin{aligned}
{\left[\Phi_{1, m}^{\left(s_{1}\right), i j}, \tilde{\Phi}_{2, n}^{\left(s_{2}\right)}\right]=} & -\sum_{r=1}^{\left[\frac{s_{1}+s_{2}+2}{2}\right]} \frac{\mathrm{i}(-1)^{r}\left(2 s_{1}-1\right)\left(2 s_{2}-1\right)}{2\left(s_{1}+s_{2}-2 r\right)+3}\left[\mathrm{BB}_{2 r,+}^{s_{1}+1, s_{2}+2}(m, n ; \mu) \Phi_{1, m+n}^{\left(s_{1}+s_{2}+2-2 r\right), i j}\right. \\
& \left.+\mathrm{BB}_{2 r,-}^{s_{1}+1, s_{2}+2}(m, n ; \mu) \Phi_{1, m+n}^{\left(s_{1}+s_{2}+2-2 r\right), i j}\right] .
\end{aligned}
$$

\section{E.3 The two (anti)commutators with common $\tilde{\Phi}_{\frac{3}{2}, \rho}^{\left(s_{1}\right), i}$}

The two kinds of (anti)commutators are given. The following anticommutator can be obtained

$$
\begin{aligned}
\left\{\tilde{\Phi}_{\frac{3}{2}, \rho}^{\left(s_{1}\right), i}, \tilde{\Phi}_{\frac{3}{2}, \omega}^{\left(s_{2}\right), j}\right\}= & -\delta^{i j} \sum_{r=1}^{\left[\frac{s_{1}+s_{2}+2}{2}\right]} \mathrm{i}(-1)^{r}\left(2 s_{1}-1\right)\left(2 s_{2}-1\right)[ \\
& \frac{2\left(s_{1}+s_{2}-r+3\right)}{(2 r-1)\left(2 s_{1}+2 s_{2}-4 r+7\right)} \mathrm{FF}_{2 r-1,-}^{s_{1}+\frac{3}{2}, s_{2}+\frac{3}{2}}(\rho, \omega ; \mu) \Phi_{0, \rho+\omega}^{\left(s_{1}+s_{2}-2 r+4\right)} \\
& +\frac{1}{2\left(s_{1}+s_{2}-2 r\right)+3}\left(\mathrm{FF}_{2 r-1,+}^{s_{1}+\frac{3}{2}, s_{2}+\frac{3}{2}}(\rho, \omega ; \mu)\right. \\
& \left.\left.+\frac{2 \nu\left(s_{1}+s_{2}+3-r\right)}{(2 r-1)\left(2 s_{1}+2 s_{2}-4 r+7\right)} \mathrm{FF}_{2 r-1,-}^{s_{1}+\frac{3}{2}, s_{2}+\frac{3}{2}}(\rho, \omega ; \mu)\right) \tilde{\Phi}_{2, \rho+\omega}^{\left(s_{1}+s_{2}-2 r+2\right)}\right] \\
& -\frac{\left[\frac{s_{1}+s_{2}+2}{2}\right]}{\sum_{r=1}^{2} \frac{\mathrm{i}(-1)^{r}\left(2 s_{1}-1\right)\left(2 s_{2}-1\right)}{2\left(s_{1}+s_{2}-2 r\right)+3}\left[\mathrm{FF}_{2 r,+}^{s_{1}+\frac{3}{2}, s_{2}+\frac{3}{2}}(\rho, \omega ; \mu) \Phi_{1, \rho+\omega}^{\left(s_{1}+s_{2}-2 r+2\right), i j}\right.} \\
& \left.+\frac{2\left(s_{1}+s_{2}-r\right)+5}{2 r} \mathrm{FF}_{2 r,-}^{\left.s_{1}+\frac{3}{2}, s_{2}+\frac{3}{2}\right)}(\rho, \omega ; \mu) \tilde{\Phi}_{1, \rho+\omega}^{\left(s_{1}+s_{2}-2 r+2\right), i j}\right],
\end{aligned}
$$

where (4.8) and (4.9) are used. 
We have the next commutator

$$
\begin{aligned}
{\left[\tilde{\Phi}_{\frac{3}{2}, \rho}^{\left(s_{1}\right), i}, \tilde{\Phi}_{2, m}^{\left(s_{2}\right)}\right]=} & \sum_{r=1}^{\left[\frac{s_{1}+s_{2}+3}{2}\right]} \mathrm{i}(-1)^{r}\left(2 s_{1}-1\right)\left(2 s_{2}-1\right)[ \\
& \frac{2\left(s_{2}-r+2\right)}{(2 r-1)\left(2 s_{1}+2 s_{2}-4 r+7\right)} \mathrm{BF}_{2 r-1,-}^{s_{2}+2, s_{1}+\frac{3}{2}}(m, \rho ; \mu) \Phi_{\frac{1}{2}, \rho+m}^{\left(s_{1}+s_{2}-2 r+4\right), i} \\
& \left.+\frac{1}{2\left(s_{1}+s_{2}-2 r\right)+3} \mathrm{BF}_{2 r,+}^{s_{2}+2, s_{1}+\frac{3}{2}}(m, \rho ; \mu) \tilde{\Phi}_{\frac{3}{2}, \rho+m}^{\left(s_{1}+s_{2}-2 r+2\right), i}\right]
\end{aligned}
$$

where (4.6) is used.

\section{E.4 The final commutator}

With (4.5), the final commutator is given by

$$
\begin{aligned}
{\left[\tilde{\Phi}_{2, m}^{\left(s_{1}\right)}, \tilde{\Phi}_{2, n}^{\left(s_{2}\right)}\right]=} & -\frac{\left[\frac{s_{1}+s_{2}+3}{2}\right]}{\sum_{r=1}^{2} \mathrm{i}(-1)^{r}\left(2 s_{1}-1\right)\left(2 s_{2}-1\right)} \\
& \times\left[\frac{1}{2\left(s_{1}+s_{2}-2 r\right)+7} \mathrm{BB}_{2 r,-}^{s_{1}+2, s_{2}+2}(m, n ; \mu) \Phi_{0, m+n}^{\left(s_{1}+s_{2}-2 r+4\right)}\right. \\
& +\frac{1}{2\left(s_{1}+s_{2}-2 r\right)+3}\left(\mathrm{BB}_{2 r,+}^{s_{1}+2, s_{2}+2}(m, n ; \mu)\right. \\
& \left.\left.+\frac{\nu}{2\left(s_{1}+s_{2}-2 r\right)+7} \mathrm{BB}_{2 r,-}^{s_{1}+2, s_{2}+2}(m, n ; \mu)\right) \tilde{\Phi}_{2, m+n}^{\left(s_{1}+s_{2}-2 r+2\right)}\right]
\end{aligned}
$$

The $\nu$ dependence appears in the coefficient.

The complete 15 (anti)commutators are given by (4.16), (4.17), (4.18), (4.19), (4.20), eqs. (E.1), (E.2), (E.3), (E.4), (E.5), (E.6), (E.7), (E.8), (E.9), and (E.10).

\section{F The $\mathcal{N}=2$ higher spin algebra $\operatorname{shs}[\lambda]$}

In this appendix, we describe the embedding of $\operatorname{sh} s[\mu]$ inside $s h s_{2}[\mu]$ by taking the algebraically obvious one where the $2 \times 2$ identity matrix is taken. Although this is accidental (not physical because the different higher spin multiplets are mixed), the nontrivial relations between the two kinds of structure constants below satisfy in general. They do not depend on the accidental or physical embeddings we take. We refer to the paper [12] for the physical embedding of $s h s[\mu]$ inside $s h s_{2}[\mu]$ and see also the footnote 4 for the corresponding $2 \times 2$ matrices.

\section{F.1 Review}

The (anti)commutation relations of the $\mathcal{N}=2$ higher spin algebra $\operatorname{sh} s[\lambda]$ generated by bosonic generators $T^{j}$ and $U^{j}$ of integer spins $(j+1)$ and fermionic generators $\Psi^{j}$ and 
$\bar{\Psi}^{j}$ of half integer spins $(j+1)$ with $j=0, \frac{1}{2}, 1, \frac{3}{2}, \cdots$ can be summarized by [32] (in the notation of [36])

$$
\begin{aligned}
& {\left[T_{m}^{j}, T_{m^{\prime}}^{j^{\prime}}\right]=\sum_{j^{\prime \prime}, m^{\prime \prime}} f_{T T T}^{j j^{\prime} j^{\prime \prime}} C_{m m^{\prime} m^{\prime \prime}}^{j j^{\prime} j^{\prime \prime}} T_{m^{\prime \prime}}^{j^{\prime \prime}},} \\
& {\left[T_{m}^{j}, \Psi_{r^{\prime}}^{j^{\prime}}\right]=\sum_{j^{\prime \prime}, r^{\prime \prime}} f_{T \Psi \Psi}^{j j^{\prime} j^{\prime \prime}} C_{m r^{\prime} r^{\prime \prime}}^{j j^{\prime} j^{\prime \prime}} \Psi_{r^{\prime \prime}}^{j^{\prime \prime}}} \\
& {\left[U_{m}^{j}, \Psi_{r^{\prime}}^{j^{\prime}}\right]=\sum_{j^{\prime \prime}, r^{\prime \prime}} f_{U \Psi \Psi}^{j j^{\prime} j^{\prime \prime}} C_{m r^{\prime} r^{\prime \prime}}^{j j^{\prime} j^{\prime \prime}} \Psi_{r^{\prime \prime}}^{j^{\prime \prime}}} \\
& \left\{\Psi_{r}^{j}, \bar{\Psi}_{r^{\prime}}^{j^{\prime}}\right\}=\sum_{j^{\prime \prime}, m^{\prime \prime}} C_{r r^{\prime} m^{\prime \prime}}^{j j^{\prime} j^{\prime \prime}}\left(f_{\Psi \bar{\Psi} T}^{j j^{\prime} j^{\prime \prime}} T_{m^{\prime \prime}}^{j^{\prime \prime}}+f_{\Psi \bar{\Psi} U}^{j j^{\prime} j^{\prime \prime}} U_{m^{\prime \prime}}^{j^{\prime \prime}}\right) . \\
& {\left[U_{m}^{j}, U_{m^{\prime}}^{j^{\prime}}\right]=\sum_{j^{\prime \prime}, m^{\prime \prime}} f_{U U U}^{j j^{\prime} j^{\prime \prime}} C_{m m^{\prime} m^{\prime \prime}}^{j j^{\prime} j^{\prime \prime}} U_{m^{\prime \prime}}^{j^{\prime \prime}}} \\
& {\left[T_{m}^{j}, \bar{\Psi}_{r^{\prime}}^{j^{\prime}}\right]=\sum_{j^{\prime \prime}, r^{\prime \prime}} f_{T \bar{\Psi}}^{j j^{\prime} j^{\prime \prime}} C_{m r^{\prime} r^{\prime \prime}}^{j j^{\prime} j^{\prime \prime}} \bar{\Psi}_{r^{\prime \prime}}^{j^{\prime \prime}}} \\
& {\left[U_{m}^{j}, \bar{\Psi}_{r^{\prime}}^{j^{\prime}}\right]=\sum_{j^{\prime \prime}, r^{\prime \prime}} f_{U \bar{\Psi}^{\prime} \bar{\Psi}}^{j j^{\prime \prime}} C_{m r^{\prime} r^{\prime \prime}}^{j j^{\prime} j^{\prime \prime}} \bar{\Psi}_{r^{\prime \prime}}^{j^{\prime \prime}}}
\end{aligned}
$$

Of course, the summation $\sum_{j^{\prime \prime}, m^{\prime \prime}}$ (or other similar ones) above is only valid for the selection rule of $3 j$-symbol in Clebsch-Gordan coefficients eq. (F.5). ${ }^{23}$ The structure constants in eq. (F.1) are given by

$$
\begin{array}{ll}
f_{T T T}^{j j^{\prime} j^{\prime \prime}}=\left(1-\epsilon^{j j^{\prime} j^{\prime \prime}}\right) F_{000}^{j j^{\prime} j^{\prime \prime}}( \pm 1 \mp \mu), & f_{U U U}^{j j^{\prime} j^{\prime \prime}}=\left(1-\epsilon^{j j^{\prime} j^{\prime \prime}}\right) F_{000}^{j j^{\prime} j^{\prime \prime}}( \pm \mu), \\
f_{T \Psi \Psi}^{j j^{\prime} j^{\prime \prime}}=-\epsilon^{j j^{\prime} j^{\prime \prime}} f_{T \bar{\Psi} \bar{\Psi}}^{j j^{\prime} j^{\prime \prime}}, & f_{T \bar{\Psi} \bar{\Psi}^{j} \bar{\Psi}}^{j j^{\prime} j^{\prime \prime}}=+F_{0 \frac{1}{2} \frac{1}{2}}^{j j^{\prime} j^{\prime \prime}}(-\mu), \\
f_{U \Psi \Psi}^{j j^{\prime} j^{\prime \prime}}=-\epsilon^{j j^{\prime} j^{\prime \prime}} f_{U \bar{\Psi} \bar{\Psi}}^{j j^{\prime} j^{\prime \prime}}, & f_{U \bar{\Psi}^{\prime} \bar{\Psi}^{\prime \prime}}^{j \bar{\Psi}^{\prime \prime}}=-\epsilon^{j j^{\prime} j^{\prime \prime}} F_{0-\frac{1}{2}-\frac{1}{2}}^{j j^{\prime} j^{\prime \prime}}(1-\mu), \\
f_{\Psi \bar{\Psi} T}^{j j^{\prime} j^{\prime \prime}}=-\epsilon^{j j^{\prime} j^{\prime \prime}} F_{\frac{1}{2}-\frac{1}{2} 0}^{j j^{\prime} j^{\prime \prime}}(1-\mu), & f_{\Psi \bar{\Psi} U}^{j j^{\prime} j^{\prime \prime}}=-F_{-\frac{1}{2} \frac{1}{2} 0}^{j j^{\prime} j^{\prime \prime}}(-\mu)
\end{array}
$$

with $\epsilon^{j j^{\prime} j^{\prime \prime}}=(-1)^{j+j^{\prime}-j^{\prime \prime}}$. The symbols $F_{l l^{\prime} l^{\prime \prime}}^{j j^{\prime \prime}}(\mu)$ in eq. (F.2) are given by [32, 36]

$$
\begin{aligned}
F_{l l^{\prime} l^{\prime \prime}}^{j j^{\prime \prime}}(\mu)= & \sqrt{2 j^{\prime \prime}+1} \Delta^{j j^{\prime} j^{\prime \prime}} \sum_{t=\max \left\{0,-j^{\prime \prime}+j+l^{\prime},-j^{\prime \prime}+j^{\prime}-l\right\}}^{\min \left\{j+j^{\prime}-j^{\prime \prime}, j-l, j^{\prime}+l^{\prime}\right\}} \\
\times & {\left[(-1)^{t} \prod_{p=1}^{j+j^{\prime}-j^{\prime \prime}-t}\left(\mu-j^{\prime \prime}+l^{\prime \prime}-p\right) \prod_{q=1}^{t}\left(\mu+j^{\prime \prime}+l^{\prime \prime}+q\right)\right.} \\
& \left.\quad \times \frac{\sqrt{(j+l) !(j-l) !\left(j^{\prime}+l^{\prime}\right) !\left(j^{\prime}-l^{\prime}\right) !\left(j^{\prime \prime}+l^{\prime \prime}\right) !\left(j^{\prime \prime}-l^{\prime \prime}\right) !}}{t !\left(j+j^{\prime}-j^{\prime \prime}-t\right) !\left(t+j^{\prime \prime}-j-l^{\prime}\right) !\left(t+j^{\prime \prime}-j^{\prime}+l\right) !(j-l-t) !\left(j^{\prime}+l^{\prime}-t\right) !}\right]
\end{aligned}
$$

where we assume $l+l^{\prime}=l^{\prime \prime}$ and

$$
\Delta^{j j^{\prime} j^{\prime \prime}} \equiv \sqrt{\frac{\left(j+j^{\prime}-j^{\prime \prime}\right) !\left(j+j^{\prime \prime}-j^{\prime}\right) !\left(j^{\prime}+j^{\prime \prime}-j\right) !}{\left(j+j^{\prime}+j^{\prime \prime}+1\right) !}} .
$$

The dummy variable $t$ runs from the maximum value among $\left(0,-j^{\prime \prime}+j+l^{\prime},-j^{\prime \prime}+j^{\prime}-l\right)$ to the minimum value among $\left(j+j^{\prime}-j^{\prime \prime}, j-l, j^{\prime}+l^{\prime}\right)$. The SL(2) Clebsch-Gordan coefficient

\footnotetext{
${ }^{23}$ One of them is given by $\left|j-j^{\prime}\right| \leq j^{\prime \prime} \leq j+j^{\prime}$.
} 
(or $3 j$ symbol) appearing in eq. (F.1) is given by

$$
\begin{aligned}
C_{m m^{\prime} m^{\prime \prime}}^{j j^{\prime} j^{\prime \prime}}= & (-1)^{j-j^{\prime}+m^{\prime \prime}} \sqrt{2 j^{\prime \prime}+1}\left(\begin{array}{ccc}
j & j^{\prime} & j^{\prime \prime} \\
m & m^{\prime} & -m^{\prime \prime}
\end{array}\right) \\
= & \Delta^{j j^{\prime} j^{\prime \prime}} \sqrt{\left(2 j^{\prime \prime}+1\right)(j+m) !(j-m) !\left(j^{\prime}+m^{\prime}\right) !\left(j^{\prime}-m^{\prime}\right) !\left(j^{\prime \prime}+m^{\prime \prime}\right) !\left(j^{\prime \prime}-m^{\prime \prime}\right) !} \\
& \times \sum_{t}\left[\frac{(-1)^{t}}{t !\left(j+j^{\prime}-j^{\prime \prime}-t\right) !(j-m-t) !\left(j^{\prime}+m^{\prime}-t\right) !}\right. \\
& \left.\times \frac{1}{\left(-j^{\prime}+j^{\prime \prime}+m+t\right) !\left(-j+j^{\prime \prime}-m^{\prime}+t\right) !}\right],
\end{aligned}
$$

where the summation is over all nonnegative integers $t$ such that the arguments in the factorials are nonnegative [67] or the SL(2) Clebsch-Gordan coefficient can be wrritten by using the hypergeometric function [28] and the expression eq. (F.4) is used.

Then the $\mathcal{N}=2$ higher spin algebra is dscribed by eq. (F.1) with eqs. (F.2), (F.3) and (F.5).

\section{F.2 How to obtain the $\mathcal{N}=2$ higher spin algebra $\operatorname{shs}[\lambda]$ from $\mathcal{N}=4$ higher spin algebra $\operatorname{shs}_{2}[\lambda]$}

According to the definition (3.9), there are no negative factorials in $N_{r}^{h_{1}, h_{2}}(m, n)$ because of $h_{1}-1 \pm m, h_{2}-1 \pm n, r-1-k \geq 0$. Then, we have [28]

$$
\begin{aligned}
& N_{r}^{h_{1}, h_{2}}(m, n)= \\
& \sum_{k=0}^{r-1} \frac{(-1)^{k}(r-1) !\left(h_{1}-1+m\right) !\left(h_{1}-1-m\right) !\left(h_{2}-1+n\right) !\left(h_{2}-1-n\right) !}{k !(r-1-k) !\left(h_{1}+m-r+k\right) !\left(h_{1}-1-m-k\right) !\left(h_{2}-1+n-k\right) !\left(h_{2}-n-r+k\right) !} .
\end{aligned}
$$

On the other hand, the SL(2) Clebsch-Gordan coefficient can be written as

$$
\begin{aligned}
& C_{m, n, m+n}^{h_{1}-1, h_{2}-1, h_{1}+h_{2}-r-1}= \\
& \Delta^{h_{1}-1, h_{2}-1, h_{1}+h_{2}-r-1} \sqrt{\left(2 h_{1}-2\right) !\left(2 h_{2}-2\right) !\left(2 h_{1}+2 h_{2}-2 r-1\right) !} \\
& \quad \times \sum_{t} \frac{(-1)^{t} \alpha_{m}^{h_{1}-1} \alpha_{n}^{h_{2}-1} \alpha_{m+n}^{h_{1}+h_{2}-r-1}}{t !(r-1-t) !\left(h_{1}-1-m-t\right) !\left(h_{2}-1+n-t\right) !\left(h_{1}+m-r+t\right) !\left(h_{2}-n-r+t\right) !} .
\end{aligned}
$$

Here we introduce the scale factor $[36]$

$$
\alpha_{m}^{j} \equiv \sqrt{\frac{(j-m) !(j+m) !}{(2 j) !}} .
$$

From eqs. (F.6) and (F.7), we arrive at

$$
\begin{aligned}
C_{m, n, m+n}^{h_{1}-1, h_{2}-1, h_{1}+h_{2}-r-1}= & \frac{\Delta^{h_{1}-1, h_{2}-1, h_{1}+h_{2}-r-1} \alpha_{m+n}^{h_{1}+h_{2}-r-1}}{(r-1) ! \alpha_{m}^{h_{1}-1} \alpha_{n}^{h_{2}-1}} \\
& \times \sqrt{\frac{\left(2 h_{1}+2 h_{2}-2 r-1\right) !}{\left(2 h_{1}-2\right) !\left(2 h_{2}-2\right) !}} N_{r}^{h_{1}, h_{2}}(m, n),
\end{aligned}
$$

where the relations eqs. (F.8) and (F.4) are used. This is nothing but (2.18) of [28] for even $r$. In our case, the above relation holds for odd $r$. This relation eq. (F.9) is only valid for the range (physical range) where Clebsch-Gordan coefficients satisfy the selection 
rules. Because $\left(1-\epsilon^{j j^{\prime} j^{\prime \prime}}\right)=\left(1-(-1)^{r-1}\right)$ in the function $F_{k k^{\prime} k^{\prime \prime}}^{h_{1}-1, h_{2}-1, h_{1}+h_{2}-r-1}(\mu)$, we can consider only even $r$ case.

Then we can arrange the nontrivial relation between the generalized hypergeometric function $\phi(4.1)$ and $F_{k k^{\prime} k^{\prime \prime}}^{j j^{\prime \prime}}$ symbol in eq. (F.3). For $k=k^{\prime}=k^{\prime \prime}=0$, we have

$$
\begin{aligned}
F_{000}^{h_{1}-1, h_{2}-1, h_{1}+h_{2}-2 r-1}(\mu)= & -\frac{2 \mathrm{i}(-1)^{r}\left(2 h_{1}-1\right)\left(2 h_{2}-1\right) t_{1}\left[h_{1}\right] t_{1}\left[h_{2}\right]}{\Delta^{h_{1}-1, h_{2}-1, h_{1}+h_{2}-2 r-1} t_{1}\left[h_{1}+h_{2}-2 r\right]\left(2 h_{1}+2 h_{2}-4 r-1\right)} \\
& \times \sqrt{\frac{\left(2 h_{1}-2\right) !\left(2 h_{2}-2\right) !}{\left(2 h_{1}+2 h_{2}-4 r-1\right) !}} \phi_{2 r}^{h_{1}, h_{2}}(-\mu, 1),
\end{aligned}
$$

where we introduce

$$
t_{1}[h] \equiv \frac{2(-1)^{2 h+1}\left(\frac{\mathrm{i}}{2}\right)^{h+1}}{(2 h-1)} \sqrt{\frac{\left[h-\frac{3}{2}\right]_{h-1}}{[h-1]_{h-1}}} .
$$

Here the falling Pochhammer symbol is used. We checked that eq. (F.10) holds for odd $r$. Other four cases of $F$ symbols will be described later in eqs. (F.19) and (F.20). According to eq. (F.3), the power of $\mu$ is given by $\left(j+j^{\prime}-j^{\prime \prime}\right)=(2 r-1)$. We observe that for the large values of $r$ closing to the sum of $h_{1}$ and $h_{2}$, there exists a factorized form (where each factor is linear in $\mu$ ) of $\mu$ in the left hand side of eq. (F.10) together with eq. (F.3). On the other hand, for the small values of $r$, there are some polynomials in $\mu$ which cannot be written in terms of factorized forms. In the region of middle values of $r$, there are some factorized forms multipled by some polynomials. In other words, there are two products in eq. (F.3) specified by dummy variables $p$ and $q$. If they have same expression with apprpriate possible values of $t$, then we will have former behavior where there exists only factorized form. If they are different from each other, then we will have latter behavior which shows some polynomials.

On the other hand, we have seen that as we increase the $r$ values which are the lower indices of generalized hypergeometric functions, the power of $\mu$ increases in the discussion of (3.11). Then how the $F$ symbols are related to the generalized hypergeometric functions? First of all, we have checked that the $\mu$ dependent parts of the former and the latter are exactly the same for the range of $h_{1}$ and $h_{2}$ we consider in this paper. The nontrivial part is to determine the $\mu$ independent numerical values appearing in an overall factors. They do depend on $h_{1}, h_{2}$ and $r$ as in eq. (F.10). We have checked the relative coefficients explicitly (Around both the total spin, $h_{1}+h_{2}$, and the spin $r$ near 10, they are determined completely) and it turns out that we have the relation between the $F$ symbols and the generalized hypergeometric functions. ${ }^{24}$

\footnotetext{
${ }^{24}$ For example, for the $h_{1}=h_{2}=100$ case, we have several values for $F$ symbols as follows. When $r=3$, the $F$ contains $\left(-\nu^{2}-2 \nu+15329555\right)$ which appears in $\phi_{3}^{100,100}(-\nu, 1)$. For $r=10$, the corresponding polynomial is given by $\left(\nu^{8}+8 \nu^{7}-18824252 \nu^{6}-112945624 \nu^{5}+80986952555566 \nu^{4}+323948563193240 \nu^{3}-\right.$ $\left.112329283433337991596 \nu^{2}-224659214764103558088 \nu+46136714396921922707472825\right)$ which is common in $\phi_{10}^{100,100}(-\nu, 1)$. For the $r=199$, we have $(-197+\nu)(-195+\nu) \cdots(-1+\nu)(3+\nu)(5+\nu) \cdots(199+\nu)$. Also we observe that $\phi_{199}^{100,100}(-\nu, 1)$ contains this factorized form. Although we do not write down the relative coefficients (the number of decimal digits are order of several hundreds) here, they satisfy the relations in eq. (F.10). Therefore, we conclude that the nontrivial relation eq. (F.10) holds for any $h_{1}$ and $h_{2}$.
} 


\section{F.2.1 The first commutator of eq. (F.1)}

We would like to construct the $\mathcal{N}=4$ higher spin generators which provide the above $\mathcal{N}=2$ higher spin algebra $\operatorname{shs}[\mu]$. In general, the spin $(j+1)$ generator consists of operator $K$ independent oscillator part and the operator $K$ dependent oscillator part. The number of oscillators is given by $2 j$ with undetermined two coefficients. Then we substitute this ansatz into the first of eq. (F.1) and use the previous relation between the oscillators (4.5) and other ones associated with the footnote 8 . We have found that the mode dependent SL(2) Clebsch-Gordan coefficient is given by eq. (F.9) and symbol $F$ is given by eq. (F.10). Then the above two coefficients can be fixed and by using (2.6) we can write down the bosonic spin $(j+1)$ generator in $\mathcal{N}=2$ higher spin algebra $s h s[\mu]$ in terms of the linear combination of two $\mathcal{N}=4$ higher spin generators.

It turns out that the $\mathcal{N}=2$ higher spin generator can be expressed in terms of the ones of $\mathcal{N}=4$ higher spin generators

$$
\begin{aligned}
\alpha_{m}^{j} T_{m}^{j} & =\underbrace{\hat{y}_{(1} \ldots \hat{y}_{1}}_{j+m} \underbrace{\hat{y}_{2} \ldots \hat{y}_{2)}}_{j-m}\left(K t_{1}[j+1](2 j+1)+t_{1}[j+1] \nu-t_{2}[j+1](2 j-3)\right) \\
& =t_{1}[j+1] \Phi_{0, m}^{(j+1)}+t_{2}[j+1] \tilde{\Phi}_{2, m}^{(j-1)}
\end{aligned}
$$

where the previous relations in (2.6) and eq. (F.11) are used and

$$
t_{2}[h] \equiv \frac{(-2 h+1+\nu)}{(2 h-5)} t_{1}[h] .
$$

There is also a linear $\nu$ dependence in the coefficient of eq. (F.12) or in eq. (F.13).

Therefore, we have the explicit realization of oscillators in eq. (F.12) which generates the first commutator of eq. (F.1).

\section{F.2.2 Other (anti)commutators of eq. (F.1)}

Other type of bosonic generator in the $\mathcal{N}=2$ higher spin algebra can be constructed similarly. As done in previous example in eq. (F.12), we make a linear combination of oscillators with two undetermined coefficients. One of them has an explicit operator $K$ dependence. After substituting this ansatz into the second commutator of eq. (F.1) and using the previous relations eqs. (F.9) and (F.10), we can determine the above two coefficients. For the quantities

$$
u_{1}[h] \equiv-t_{1}[h], \quad u_{2}[h] \equiv \frac{(-2 h+1-\nu)}{(2 h-5)} t_{1}[h],
$$

the corresponding $\mathcal{N}=2$ higher spin generator is given by either the oscillators or the ones of $\mathcal{N}=4$ higher spin generators

$$
\begin{aligned}
\alpha_{m}^{j} U_{m}^{j} & =\underbrace{\hat{y}_{(1} \ldots \hat{y}_{1}}_{j+m} \underbrace{\hat{y}_{2} \ldots \hat{y}_{2)}}_{j-m}\left(K u_{1}[j+1](2 j+1)+u_{1}[j+1] \nu-u_{2}[j+1](2 j-3)\right) \\
& =u_{1}[j+1] \Phi_{0, m}^{(j+1)}+u_{2}[j+1] \tilde{\Phi}_{2, m}^{(j-1)} .
\end{aligned}
$$


There is also a linear $\nu$ dependence in the coefficient of eq. (F.15) or in eq. (F.14). Therefore, the explicit realization of oscillators is given by eq. (F.15) which generates the second commutator of eq. (F.1).

Similarly, together with

$$
\begin{array}{ll}
\psi_{1}[h] & \equiv \frac{\mathrm{i}^{h-\frac{1}{2}}}{2^{h+1}} \sqrt{\frac{[h-2]_{h-\frac{3}{2}}}{(h-1)\left[h-\frac{1}{2}\right]_{h-\frac{3}{2}}}}, \quad \psi_{2}[h] \equiv \mathrm{i} \frac{(h-1)}{(h-2)} \psi_{1}[h], \\
\bar{\psi}_{1}[h] & =\psi_{1}[h], \quad \bar{\psi}_{2}[h] \equiv-\psi_{2}[h],
\end{array}
$$

the remaining $\mathcal{N}=2$ higher spin generators

$$
\begin{aligned}
\alpha_{\rho}^{j} \Psi_{\rho}^{j} & =-2 e^{\frac{\mathrm{i} \pi}{4}} \underbrace{\hat{y}_{(1} \ldots \hat{y}_{1}}_{j+\rho} \underbrace{\hat{y}_{2} \ldots \hat{y}_{2)}}_{j-\rho}\left(K \mathrm{i} \psi_{1}[j+1](j)+\psi_{2}[j+1](j-1)\right) \\
& =\psi_{1}[j+1] \Phi_{\frac{1}{2}, \rho}^{\left(j+\frac{1}{2}\right)}+\psi_{2}[j+1] \tilde{\Phi}_{\frac{3}{2}, \rho}^{\left(j-\frac{1}{2}\right)}, \\
\alpha_{\rho}^{j} \bar{\Psi}_{\rho}^{j} & =-2 e^{\frac{i \pi}{4}} \underbrace{\hat{y}_{\left(1 \ldots \hat{y}_{1}\right.}}_{j+\rho} \underbrace{\hat{y}_{2} \ldots \hat{y}_{2)}}_{j-\rho}\left(K \mathrm{i} \bar{\psi}_{1}[j+1](j)+\bar{\psi}_{2}[j+1](j-1)\right) \\
& =\bar{\psi}_{1}[j+1] \Phi_{\frac{1}{2}, \rho}^{\left(j+\frac{1}{2}\right)}+\bar{\psi}_{2}[j+1] \tilde{\Phi}_{\frac{3}{2}, \rho}^{\left(j-\frac{1}{2}\right)}
\end{aligned}
$$

provide the remaining five (anti)commutators of eq. (F.1).

Then the four $\mathcal{N}=2$ higher spin generators are given by eqs. (F.12), (F.15) and (F.17) in terms of oscillators or the ones in $\mathcal{N}=4$ higher spin generators which contain the $2 \times 2$ identity matrix in (2.6).

\section{F.3 The (anti)commutators of oscillators by using the structure constants in $\operatorname{shs}[\mu]$}

Note that we can present the three cases (4.5), (4.6), (4.7), (4.8) and (4.9) studied in previous sections by using the structure constants in the $\mathcal{N}=2$ higher spin algebra $s h s[\mu]$ of eq. (F.1). For example, by using eqs. (F.12) and (F.15) in order to remove the operator $K$ dependent oscillator term and using the first two relations of eq. (F.1), we rewrite the left side of (4.5) as follows:

$$
\begin{aligned}
& \quad[\underbrace{\hat{y}_{(1} \ldots \hat{y}_{1}}_{h_{1}-1+m} \underbrace{\left.\hat{y}_{2} \ldots \hat{y}_{2}\right)}_{h_{1}-1-m}, \underbrace{\hat{y}_{(1} \ldots \hat{y}_{1}}_{h_{2}-1+n} \underbrace{\left.\hat{y}_{2} \ldots \hat{y}_{2}\right)}_{h_{2}-1-n}] \\
& =\sum_{h=\left|h_{1}-h_{2}\right|+1}^{h_{1}+h_{2}-1} \frac{\alpha_{m}^{h_{1}-1} \alpha_{n}^{h_{2}-1}}{4\left(2 h_{1}-1\right)\left(2 h_{2}-1\right) t_{1}\left[h_{1}\right] t_{1}\left[h_{2}\right]} \frac{(2 h-1) t_{1}[h]}{\alpha_{m+n}^{h-1}} C_{m, n, m+n}^{h_{1}-1, h_{2}-1, h-1} \\
& \quad \times[\left(f_{T T T}^{h_{1}-1, h_{2}-1, h-1}+f_{U U U}^{h_{1}-1, h_{2}-1, h-1}\right) \underbrace{\hat{y}_{(1} \ldots \ldots \hat{y}_{1}}_{h-1+m+n} \underbrace{\hat{y}_{2} \ldots \ldots \hat{y}_{2)}}_{h-1-m-n} \\
& \quad+\left(f_{T T T}^{h_{1}-1, h_{2}-1, h-1}-f_{U U U}^{h_{1}-1, h_{2}-1, h-1}\right) \underbrace{\hat{y}_{(1} \ldots \ldots \hat{y}_{1}}_{h-1+m+n} \underbrace{\left.\hat{y}_{2} \ldots \ldots \hat{y}_{2}\right)}_{h-1-m-n} K],
\end{aligned}
$$

where eqs. (F.8), (F.11), (F.7), (F.2) and (F.3) are used. Other remaining (anti)commutators are presented in appendix $\mathrm{G}$. 


\section{F.3.1 The structure constants in the $\operatorname{sh} s[\mu]$ by using the generalized hyper- geometric functions}

There are two different expressions for the same quantity. For example, the right hand side of eq. (F.18) written in terms of the structure constants in $s h s[\mu]$ should be equal to the right hand side of (4.5) written in terms of the ones in $s h s_{2}[\mu]$. This leads to the previous relation in eq. (F.10). There are also other nontrivial relations which can be obtained from (4.6), (4.7), (4.8) and (4.9) and the corresponding ones in appendix G as follows. Four different types of $F$ symbols are ${ }^{25}$

$$
\begin{aligned}
& F_{0 \frac{1}{2} \frac{1}{2}}^{h_{1}-1, h_{2}-\frac{1}{2}, h_{1}+h_{2}-r-\frac{1}{2}}(-\mu) \pm F_{0-\frac{1}{2}-\frac{1}{2}}^{h_{1}-1, h_{2}-\frac{1}{2}, h_{1}+h_{2}-r-\frac{1}{2}}(1-\mu) \\
& =-\frac{\mathrm{i}^{r+1}\left(2 h_{1}-1\right)\left(2 h_{2}-1\right) t_{1}\left[h_{1}\right] \psi_{1}\left[h_{2}+\frac{1}{2}\right]}{\Delta^{h_{1}-1, h_{2}-\frac{1}{2}, h_{1}+h_{2}-r-\frac{1}{2}} \psi_{1}\left[h_{1}+h_{2}-r+\frac{1}{2}\right]\left(h_{1}+h_{2}-r-\frac{1}{2}\right)} \sqrt{\frac{\left(2 h_{1}-2\right) !\left(2 h_{2}-1\right) !}{\left(2 h_{1}+2 h_{2}-2 r\right) !}} \\
& \quad \times \frac{\left(h_{1}-\frac{1}{2}\right) \mp\left(h_{1}-r-\frac{1}{2}\right)}{r}\left[\phi_{r+1}^{h_{1}, h_{2}+1}\left(\mu, \frac{3 \pm 1}{2}\right) \pm \phi_{r+1}^{h_{1}, h_{2}+1}\left(1-\mu, \frac{3 \pm 1}{2}\right)\right], \quad \text { (F.19) }
\end{aligned}
$$

and

$$
\begin{aligned}
& F_{-\frac{1}{2} \frac{1}{2} 0}^{h_{1}-\frac{1}{2}, h_{2}-\frac{1}{2}, h_{1}+h_{2}-r}(-\mu) \pm(-1)^{r} F_{\frac{1}{2}-\frac{1}{2} 0}^{h_{1}-\frac{1}{2}, h_{2}-\frac{1}{2}, h_{1}+h_{2}-r}(1-\mu)= \\
& -\frac{2 \mathrm{i}^{r}\left(2 h_{1}-1\right)\left(2 h_{2}-1\right) \psi_{1}\left[h_{1}+\frac{1}{2}\right] \psi_{1}\left[h_{2}+\frac{1}{2}\right]}{\Delta^{h_{1}-\frac{1}{2}, h_{2}-\frac{1}{2}, h_{1}+h_{2}-r} t_{1}\left[h_{1}+h_{2}-r+1\right]\left(2 h_{1}+2 h_{2}-2 r+1\right)} \sqrt{\frac{\left(2 h_{1}-1\right) !\left(2 h_{2}-1\right) !}{\left(2 h_{1}+2 h_{2}-2 r+1\right) !}} \\
& \times \frac{\left(h_{1}+h_{2}+\frac{1}{2}\right) \mp(-1)^{r}\left(h_{1}+h_{2}+\frac{1}{2}-r\right)}{r} \\
& \times\left[\phi_{r+1}^{h_{1}+1, h_{2}+1}\left(\mu, \frac{3 \pm(-1)^{r}}{2}\right) \pm(-1)^{r} \phi_{r+1}^{h_{1}+1, h_{2}+1}\left(1-\mu, \frac{3 \pm(-1)^{r}}{2}\right)\right],
\end{aligned}
$$

where the relations eqs. (F.11), (F.16), (F.4) and (4.1) are used. Each $F$ symbols is a linear combination of generalized hypergeometric functions because the relative coefficients are different in eqs. (F.19) and (F.20), compared to the one eq. (F.10).

Therefore, we have determined the structure constants, eqs. (F.10), (F.19) and (F.20), in the $\mathcal{N}=2$ higher spin algebra $\operatorname{shs}[\mu]$ in terms of those in the $\mathcal{N}=4$ higher spin algebra $s h s_{2}[\mu]$.

\footnotetext{
${ }^{25}$ As done before, for the $h_{1}=h_{2}=100$ case, we have several values for the left hand side with plus sign of eq. (F.19) as follows. When $r=3$, the linear combination of $F$ contains $\left(\nu^{2}-15485185\right)$ which appears in the linear combination of generalized hypergeometric functions in eq. (F.19). For $r=10$, the corresponding polynomial is given by $\left(\nu^{8}-19021364 \nu^{6}+82683010446934 \nu^{4}-115857948630733805076 \nu^{2}+\right.$ 48069142225065249313021425) which appears also in the corresponding generalized hypergeometric functions. For the $r=199$, we have $(-100+\nu)(-99+\nu) \cdots(-2+\nu)(1+\nu)(2+\nu) \cdots(98+\nu)(99+\nu)$. Also we observe that the corresponding generalized hypergeometric functions contain this factorized form. Therefore, we conclude that the nontrivial relation eq. (F.19) holds for any $h_{1}$ and $h_{2}$. Similarly, if we take the plus sign of eq. (F.20), the left hand sides produce the following polynomials, $\nu$, $\left(3 \nu^{8}-57959612 \nu^{6}+255874861516642 \nu^{4}-364108448995236058268 \nu^{2}+153402332060210496539980275\right)$ and $(-199+\nu)(-197+\nu) \cdots(-5+\nu) \nu(5+\nu)(7+\nu) \cdots(197+\nu)(199+\nu)$ respectively. After obtaining the nontrivial relative coefficients by varying $h_{1}, h_{2}$ and $r$, the nontrivial relation eq. (F.20) is satisfied for any $h_{1}$ and $h_{2}$.
} 


\section{F.3.2 The relations between the structure constants in the $s h s_{2}[\mu]$ and those in the $\operatorname{sh} s[\mu]$}

For convenience, we present the structure constants (4.2) in terms of the ones in $\mathcal{N}=2$ higher spin algebra as follows:

$$
\begin{aligned}
& \mathrm{BB}_{r, \pm}^{h_{1}, h_{2}}(m, n ; \mu)= \\
& (-\mathrm{i})^{r+1} \frac{\left(h_{1}+h_{2}-r-\frac{1}{2}\right) t_{1}\left[h_{1}+h_{2}-r\right] \alpha_{m}^{h_{1}-1} \alpha_{n}^{h_{2}-1}}{\left(2 h_{1}-1\right)\left(2 h_{2}-1\right) t_{1}\left[h_{1}\right] t_{1}\left[h_{2}\right] \alpha_{m+n}^{h_{1}+h_{2}-r-1}} \\
& \times\left[F_{000}^{h_{1}-1, h_{2}-1, h_{1}+h_{2}-r-1}(-\mu) \pm F_{000}^{h_{1}-1, h_{2}-1, h_{1}+h_{2}-r-1}(1-\mu)\right] C_{m, n, m+n}^{h_{1}-1, h_{2}-1, h_{1}+h_{2}-r-1}, \\
& \mathrm{BF}_{r, \pm}^{h_{1}, h_{2}+\frac{1}{2}}(m, \rho ; \mu)= \\
& (-\mathrm{i})^{r+1} \frac{r\left(h_{1}+h_{2}-r-\frac{1}{2}\right) \psi_{1}\left[h_{1}+h_{2}-r+\frac{1}{2}\right] \alpha_{m}^{h_{1}-1} \alpha_{\rho}^{h_{2}-\frac{1}{2}}}{\left(2 h_{1}-1\right)\left(2 h_{2}-1\right) t_{1}\left[h_{1}\right] \psi_{1}\left[h_{2}+\frac{1}{2}\right] \alpha_{m+\rho}^{h_{1}+h_{2}-r-\frac{1}{2}}} \frac{1}{\left(\left(h_{1}-\frac{1}{2}\right) \mp\left(h_{1}-r-\frac{1}{2}\right)\right)} \\
& \times\left[F_{0}^{h_{1}-1, h_{2} \frac{1}{2}} \frac{1}{2}, h_{1}+h_{2}-r-\frac{1}{2}(-\mu) \pm F_{0-\frac{1}{2}-\frac{1}{2}}^{h_{1}-1, h_{2}-\frac{1}{2}, h_{1}+h_{2}-r-\frac{1}{2}}(1-\mu)\right] C_{m, \rho, m+\rho}^{h_{1}-1, h_{2}-\frac{1}{2}, h_{1}+h_{2}-r-\frac{1}{2}}, \\
& \mathrm{FF}_{r, \pm(-1)^{r}}^{h_{1}+\frac{1}{2}, h_{2}+\frac{1}{2}}(\rho, \omega ; \mu)= \\
& (-\mathrm{i})^{r} \frac{r\left(h_{1}+h_{2}-r+\frac{1}{2}\right) t_{1}\left[h_{1}+h_{2}-r+1\right] \alpha_{\rho}^{h_{1}-\frac{1}{2}} \alpha_{\omega}^{h_{2}-\frac{1}{2}}}{\left(2 h_{1}-1\right)\left(2 h_{2}-1\right) \psi_{1}\left[h_{1}+\frac{1}{2}\right] \psi_{1}\left[h_{2}+\frac{1}{2}\right] \alpha_{\rho+\omega}^{h_{1}+h_{2}-r}} \\
& \times \frac{1}{\left(\left(h_{1}+h_{2}+\frac{1}{2}\right) \mp(-1)^{r}\left(h_{1}+h_{2}+\frac{1}{2}-r\right)\right)} \\
& \times\left[F_{-\frac{1}{2} \frac{1}{2} 0}^{h_{1}-\frac{1}{2}, h_{2}-\frac{1}{2}, h_{1}+h_{2}-r}(-\mu) \pm(-1)^{r} F_{\frac{1}{2}-\frac{1}{2} 0}^{h_{1}-\frac{1}{2}, h_{2}-\frac{1}{2}, h_{1}+h_{2}-r}(1-\mu)\right] C_{\rho, \omega, \rho+\omega}^{h_{1}-\frac{1}{2}, h_{2}-\frac{1}{2}, h_{1}+h_{2}-r},
\end{aligned}
$$

where the inverse relations of eqs. (F.10), (F.19) and (F.20) together with eq. (F.9) and (4.2) are used in eq. (F.21). ${ }^{26}$

This implies that we can write down the previous $\mathcal{N}=4$ higher spin algebra $s h s_{2}[\mu]$ by using the structure constants of $\mathcal{N}=2$ higher spin algebra $\operatorname{sh} s[\mu] .{ }^{27}$

\footnotetext{
${ }^{26}$ The three upper indices of $F$ stand for the spins of the generators in the left hand side and the right hand side of the (anti)commutators respectively. In other words, the first two indices correspond to those of structure constants of (4.2) and the third one corresponds to the difference between the upper indices and lower indices in the structure constants in (4.2).

${ }^{27}$ Note that in eqs. (F.10), (F.19), (F.20) and (F.21), we have checked them for $h_{1}, h_{2} \leq 100$. Moreover, all the computations in appendices $\mathrm{F}$ and $\mathrm{G}$ are done under this restriction.
} 


\section{G The (anti)commutators of oscillators by using the structure constants in $\mathcal{N}=2$ higher spin algebra $\operatorname{shs}[\mu]$}

In this appendix, we have considered the spins $h_{1}, h_{2} \leq 100$. The left hand side of (4.6) can be expressed as

$$
\begin{aligned}
& \quad[\underbrace{\hat{y}_{(1} \ldots \hat{y}_{1}}_{h_{1}-1+m} \underbrace{\hat{y}_{2} \ldots \hat{y}_{2}}_{h_{1}-1-m}, \underbrace{\hat{y}_{(1} \ldots \hat{y}_{1}}_{h_{2}-\frac{1}{2}+\rho} \underbrace{\left.\hat{y}_{2} \ldots \hat{y}_{2}\right)}_{h_{2}-\frac{1}{2}-\rho}] \\
& =-\mathrm{i} \sum_{h=\left|h_{1}-h_{2}-\frac{1}{2}\right|}^{h_{1}+h_{2}-\frac{3}{2}} \frac{2 h \psi_{1}[h+1] \alpha_{m}^{h_{1}-1} \alpha_{\rho}^{h_{2}-\frac{1}{2}}}{2\left(2 h_{1}-5\right)\left(2 h_{2}-3\right)\left(t_{2}\left[h_{1}\right]+u_{2}\left[h_{1}\right]\right) \psi_{2}\left[h_{2}+\frac{1}{2}\right] \alpha_{m+\rho}^{h}} C_{m, \rho, m+\rho}^{h_{1}-1, h_{2}-\frac{1}{2}, h} \\
& \quad \times[\left(f_{T \Psi \Psi}^{h_{1}-1, h_{2}-\frac{1}{2}, h}+f_{U \Psi \Psi}^{h_{1}-1, h_{2}-\frac{1}{2}, h}+f_{T \bar{\Psi} \bar{\Psi}}^{h_{1}-1, h_{2}-\frac{1}{2}, h}+f_{U \bar{\Psi} \bar{\Psi}}^{h_{1}-1, h_{2}-\frac{1}{2}, h}\right) \underbrace{\hat{y}_{(1} \ldots \hat{y}_{1}}_{h+m+\rho} \underbrace{\hat{y}_{2} \ldots \hat{y}_{2)}}_{h-m-\rho} \\
& \quad+\left(f_{T \Psi \Psi}^{h_{1}-1, h_{2}-\frac{1}{2}, h}+f_{U \Psi \Psi}^{h_{1}-1, h_{2}-\frac{1}{2}, h}-f_{T \bar{\Psi} \bar{\Psi}}^{h_{1}-1, h_{2}-\frac{1}{2}, h}-f_{U \bar{\Psi} \bar{\Psi}}^{h_{1}-1, h_{2}-\frac{1}{2}, h}\right) \underbrace{\hat{y}_{(1} \ldots \hat{y}_{1}}_{h+m+\rho} \underbrace{\hat{y}_{2} \ldots \hat{y}_{2)}}_{h-m-\rho} K],
\end{aligned}
$$

where the relations eqs. (F.2), (F.7), (F.8), (F.11), (F.13), (F.14), and (F.16) are used.

The left hand side of (4.7) can be described by

$$
\begin{aligned}
& {[\underbrace{\hat{y}_{(1 \ldots} \hat{y}_{1}}_{h_{1}-1+m} \underbrace{\hat{y}_{2} \ldots \hat{y}_{2}}_{h_{1}-1-m} K, \underbrace{\hat{y}_{(1} \ldots \hat{y}_{1}}_{h_{2}-\frac{1}{2}+\rho} \underbrace{\left.\hat{y}_{2} \ldots \hat{y}_{2}\right)}_{h_{2}-\frac{1}{2}-\rho}] } \\
= & \mathrm{i} \sum_{h=\left|h_{1}-h_{2}-\frac{1}{2}\right|}^{h_{1}+h_{2}-\frac{3}{2}} \frac{2 h \psi_{1}[h+1] \alpha_{m}^{h_{1}-1} \alpha_{\rho}^{h_{2}-\frac{1}{2}}}{2\left(2 h_{1}-1\right)\left(2 h_{2}-3\right)\left(t_{1}\left[h_{1}\right]-u_{1}\left[h_{1}\right]\right) \psi_{2}\left[h_{2}+\frac{1}{2}\right] \alpha_{m+\rho}^{h}} C_{m, \rho, m+\rho}^{h_{1}-1, h_{2}-\frac{1}{2}, h} \\
& \times[\left(f_{T \Psi \Psi}^{h_{1}-1, h_{2}-\frac{1}{2}, h}-f_{U \Psi \Psi}^{h_{1}-1, h_{2}-\frac{1}{2}, h}+f_{T \bar{\Psi}}^{h_{1}-1, h_{2}-\frac{1}{2}, h}-f_{U \bar{\Psi} \bar{\Psi}}^{h_{1}-1, h_{2}-\frac{1}{2}, h}\right) \underbrace{\hat{y}_{(1 \ldots} \ldots \hat{y}_{1}}_{h+m+\rho} \underbrace{\hat{y}_{2} \ldots \hat{y}_{2)}}_{h-m-\rho} \\
& +\left(f_{T \Psi \Psi}^{h_{1}-1, h_{2}-\frac{1}{2}, h}-f_{U \Psi \Psi}^{h_{1}-1, h_{2}-\frac{1}{2}, h}-f_{T \bar{\Psi} \bar{\Psi}}^{h_{1}-1, h_{2}-\frac{1}{2}, h}+f_{U \bar{\Psi} \bar{\Psi}}^{h_{1}-1, h_{2}-\frac{1}{2}, h}\right) \underbrace{\hat{y}_{(1} \ldots \hat{y}_{1}}_{h+m+\rho} \underbrace{\hat{y}_{2} \ldots \hat{y}_{2}}_{h-m-\rho} K] .
\end{aligned}
$$

The left hand side of (4.8) can be written in terms of

$$
\begin{aligned}
\{\underbrace{\hat{y}_{(1} \ldots \hat{y}_{1}}_{h_{1}-\frac{1}{2}+\rho} \underbrace{\hat{y}_{2} \ldots \hat{y}_{2)}}_{h_{1}-\frac{1}{2}-\rho}, \underbrace{\hat{y}_{(1} \ldots \hat{y}_{1}}_{h_{2}-\frac{1}{2}+\omega} \underbrace{\left.\hat{y}_{2} \ldots \hat{y}_{2}\right)}_{h_{2}-\frac{1}{2}-\omega}\} \\
=\mathrm{i} \sum_{h=\left|h_{1}-h_{2}\right|}^{h_{1}+h_{2}-1} \frac{\left(1-(-1)^{h_{1}+h_{2}+h}\right)(2 h+1) \alpha_{\rho}^{h_{1}-\frac{1}{2}} \alpha_{\omega}^{h_{2}-\frac{1}{2}} t_{1}[h+1]}{16\left(h_{1}-\frac{3}{2}\right)\left(h_{2}-\frac{3}{2}\right) \psi_{2}\left[h_{1}+\frac{1}{2}\right] \psi_{2}\left[h_{2}+\frac{1}{2}\right] \alpha_{\rho+\omega}^{h}} C_{\rho, \omega, \rho+\omega}^{h_{1}-\frac{1}{2}, h_{2}-\frac{1}{2}, h} \\
\quad \times[\left(f_{\Psi \bar{\Psi} T}^{h_{1}-\frac{1}{2}, h_{2}-\frac{1}{2}, h}+f_{\Psi \bar{\Psi} U}^{h_{1}-\frac{1}{2}, h_{2}-\frac{1}{2}, h}\right) \underbrace{\hat{y}_{(1} \ldots \hat{y}_{1}}_{h+\rho+\omega} \underbrace{\left.\hat{y}_{2} \ldots \hat{y}_{2}\right)}_{h-\rho-\omega} \\
\quad+\left(f_{\Psi \bar{\Psi} T}^{h_{1}-\frac{1}{2}, h_{2}-\frac{1}{2}, h}-f_{\Psi \bar{\Psi} U}^{h_{1}-\frac{1}{2}, h_{2}-\frac{1}{2}, h}\right) \underbrace{\hat{y}_{(1} \ldots \hat{y}_{1}}_{h+\rho+\omega} \underbrace{\left.\hat{y}_{2} \ldots \hat{y}_{2}\right)}_{h-\rho-\omega} K] .
\end{aligned}
$$


Finally, the left hand side of (4.9) can be written as

$$
\begin{aligned}
& \{\underbrace{\hat{y}_{(1} \ldots \hat{y}_{1}}_{h_{1}-\frac{1}{2}+\rho} \underbrace{\hat{y}_{2} \ldots \hat{y}_{2)}}_{h_{1}-\frac{1}{2}-\rho}, \underbrace{\hat{y}_{(1} \ldots \hat{y}_{1}}_{h_{2}-\frac{1}{2}+\omega} \underbrace{\left.\hat{y}_{2} \ldots \hat{y}_{2}\right)}_{h_{2}-\frac{1}{2}-\omega} K\} \\
& =-\sum_{h=\left|h_{1}-h_{2}\right|}^{h_{1}+h_{2}-1} \frac{\left(1+(-1)^{h_{1}+h_{2}+h}\right)(2 h+1) \alpha_{\rho}^{h_{1}-\frac{1}{2}} \alpha_{\omega}}{16\left(h_{1}-\frac{3}{2}\right)\left(h_{2}-\frac{1}{2}\right) \psi_{2}\left[h_{1}+\frac{1}{2}\right] \psi_{1}\left[h_{2}+\frac{1}{2}\right] \alpha_{\rho+\omega}[h+1]} C_{\rho, \omega, \rho+\omega}^{h_{2}-\frac{1}{2}, h_{2}-\frac{1}{2}, h} \\
& \quad \times[\left(f_{\Psi \bar{\Psi} T}^{h_{1}-\frac{1}{2}, h_{2}-\frac{1}{2}, h}+f_{\Psi \bar{\Psi} U}^{h_{1}-\frac{1}{2}, h_{2}-\frac{1}{2}, h}\right) \underbrace{\hat{y}_{(1} \ldots \hat{y}_{1}}_{h+\rho+\omega} \underbrace{\left.\hat{y}_{2} \ldots \hat{y}_{2}\right)}_{h-\rho-\omega} \\
& \\
& \quad+\left(f_{\Psi \bar{\Psi} T}^{h_{1}-\frac{1}{2}, h_{2}-\frac{1}{2}, h}-f_{\Psi \bar{\Psi} U}^{h_{1}-\frac{1}{2}, h_{2}-\frac{1}{2}, h}\right) \underbrace{\hat{y}_{(1} \ldots \hat{y}_{1}}_{h+\rho+\omega} \underbrace{\left.\hat{y}_{2} \ldots \hat{y}_{2}\right)}_{h-\rho-\omega} K] .
\end{aligned}
$$

Therefore, we have five relations, eq. (F.18), and eqs. (G.1), (G.2), (G.3), and (G.4).

Open Access. This article is distributed under the terms of the Creative Commons Attribution License (CC-BY 4.0), which permits any use, distribution and reproduction in any medium, provided the original author(s) and source are credited.

\section{References}

[1] M.R. Gaberdiel and R. Gopakumar, Large N=4 Holography, JHEP 09 (2013) 036 [arXiv: 1305.4181] [INSPIRE].

[2] S.F. Prokushkin and M.A. Vasiliev, Higher spin gauge interactions for massive matter fields in 3-D AdS space-time, Nucl. Phys. B 545 (1999) 385 [hep-th/9806236] [InSPIRE].

[3] S. Prokushkin and M.A. Vasiliev, 3-D higher spin gauge theories with matter, hep-th/9812242 [INSPIRE].

[4] M.R. Gaberdiel, R. Gopakumar and C. Hull, Stringy AdS $S_{3}$ from the worldsheet, JHEP 07 (2017) 090 [arXiv: 1704.08665] [INSPIRE].

[5] K. Ferreira, M.R. Gaberdiel and J.I. Jottar, Higher spins on AdS 3 from the worldsheet, JHEP 07 (2017) 131 [arXiv:1704.08667] [INSPIRE].

[6] L. Eberhardt, M.R. Gaberdiel and W. Li, A holographic dual for string theory on $A d S_{3} \times S^{3} \times S^{3} \times S^{1}, J H E P 08$ (2017) 111 [arXiv:1707.02705] [INSPIRE].

[7] G. Giribet, C. Hull, M. Kleban, M. Porrati and E. Rabinovici, Superstrings on AdS $S_{3}$ at $\|=$ 1, JHEP 08 (2018) 204 [arXiv: 1803.04420] [INSPIRE].

[8] M.R. Gaberdiel and R. Gopakumar, Tensionless string spectra on AdS $S_{3}$, JHEP 05 (2018) 085 [arXiv: 1803.04423] [INSPIRE].

[9] C. Ahn and M.H. Kim, The operator product expansion between the 16 lowest higher spin currents in the $\mathcal{N}=4$ superspace, Eur. Phys. J. C 76 (2016) 389 [arXiv:1509.01908] [INSPIRE].

[10] C. Ahn, D.-g. Kim and M.H. Kim, The next 16 higher spin currents and three-point functions in the large $\mathcal{N}=4$ holography, Eur. Phys. J. C 77 (2017) 523 [arXiv:1703.01744] [InSPIRE]. 
[11] C. Ahn, D.-g. Kim and M.H. Kim, The $\mathcal{N}=4$ coset model and the higher spin algebra, Int. J. Mod. Phys. A 35 (2020) 2050046 [arXiv: 1910.02183] [InSPIRE].

[12] L. Eberhardt, M.R. Gaberdiel and I. Rienacker, Higher spin algebras and large $\mathcal{N}=4$ holography, JHEP 03 (2018) 097 [arXiv: 1801.00806] [INSPIRE].

[13] C. Ahn, Higher Spin Currents in Wolf Space. Part I, JHEP 03 (2014) 091 [arXiv: 1311.6205] [INSPIRE].

[14] M.R. Gaberdiel and C. Peng, The symmetry of large $\mathcal{N}=4$ holography, JHEP 05 (2014) 152 [arXiv: 1403.2396] [INSPIRE].

[15] M. Beccaria, C. Candu and M.R. Gaberdiel, The large $N=4$ superconformal $W_{\infty}$ algebra, JHEP 06 (2014) 117 [arXiv:1404.1694] [INSPIRE].

[16] C. Ahn, M.R. Gaberdiel and M.H. Kim, The small $\mathcal{N}=4$ superconformal $\mathcal{W}_{\infty}$ algebra, J. Phys. A 53 (2020) 395401 [arXiv: 2004.07439] [InSPIRE].

[17] C. Ahn, Higher Spin Currents in Wolf Space: Part II, Class. Quant. Grav. 32 (2015) 015023 [arXiv: 1408.0655] [INSPIRE].

[18] C. Ahn and H. Kim, Higher Spin Currents in Wolf Space for Generic N, JHEP 12 (2014) 109 [arXiv:1411.0356] [INSPIRE].

[19] C. Ahn, Higher spin currents in Wolf space: III, Class. Quant. Grav. 32 (2015) 185001 [arXiv: 1504.00070] [INSPIRE].

[20] T. Creutzig, Y. Hikida and P.B. Ronne, Extended higher spin holography and Grassmannian models, JHEP 11 (2013) 038 [arXiv: 1306. 0466] [INSPIRE].

[21] M.R. Gaberdiel, K. Jin and W. Li, Perturbations of W(infinity) CFTs, JHEP 10 (2013) 162 [arXiv: 1307.4087] [INSPIRE].

[22] M.R. Gaberdiel and T. Hartman, Symmetries of Holographic Minimal Models, JHEP 05 (2011) 031 [arXiv:1101.2910] [INSPIRE].

[23] I. Bakas and E. Kiritsis, Bosonic Realization of a Universal $W$-Algebra and $Z_{\infty}$ Parafermions, Nucl. Phys. B 343 (1990) 185 [Erratum ibid. 350 (1991) 512] [INSPIRE].

[24] T. Procházka, Exploring $\mathcal{W}_{\infty}$ in the quadratic basis, JHEP 09 (2015) 116 [arXiv:1411.7697] [INSPIRE].

[25] S. Odake, Unitary representations of $\left.W_{\infty}\right)$ algebras, Int. J. Mod. Phys. A 7 (1992) 6339 [hep-th/9111058] [INSPIRE].

[26] I. Bakas and E. Kiritsis, Grassmannian Coset Models and Unitary Representations of W(infinity), Mod. Phys. Lett. A 5 (1990) 2039 [InSPIRE].

[27] S. Odake and T. Sano, $W_{1+\infty}$ and super- $W_{\infty}$ algebras with $\mathrm{SU}(N)$ symmetry, Phys. Lett. $B$ 258 (1991) 369 [INSPIRE].

[28] C.N. Pope, L.J. Romans and X. Shen, $W_{\infty}$ and the Racah-wigner Algebra, Nucl. Phys. B 339 (1990) 191 [INSPIRE].

[29] C.N. Pope, L.J. Romans and X. Shen, The Complete Structure of $W_{\infty}$, Phys. Lett. B 236 (1990) 173 [INSPIRE].

[30] C.N. Pope, L.J. Romans and X. Shen, A New Higher Spin Algebra and the Lone Star Product, Phys. Lett. B 242 (1990) 401 [InSPIRE]. 
[31] E. Bergshoeff, C.N. Pope, L.J. Romans, E. Sezgin and X. Shen, The Super $W_{\infty}$ Algebra, Phys. Lett. B 245 (1990) 447 [INSPIRE].

[32] E.S. Fradkin and V.Y. Linetsky, Supersymmetric Racah basis, family of infinite dimensional superalgebras, SU(infinity + 1/infinity) and related 2-D models, Mod. Phys. Lett. A 6 (1991) 617 [INSPIRE].

[33] A.V. Korybut, Star product for deformed oscillator algebra Aq $(2, \nu)$, arXiv: 2006.01622 [INSPIRE].

[34] A.V. Korybut, Covariant structure constants for a deformed oscillator algebra, Theor. Math. Phys. 193 (2017) 1409 [arXiv: 1409.8634] [INSPIRE].

[35] T. Basile, N. Boulanger and F. Buisseret, Structure constants of shs $[\lambda]$ : the deformed-oscillator point of view, J. Phys. A 51 (2018) 025201 [arXiv:1604.04510] [INSPIRE].

[36] C. Candu and M.R. Gaberdiel, Duality in $N=2$ Minimal Model Holography, JHEP 02 (2013) 070 [arXiv: 1207.6646] [INSPIRE].

[37] T. Creutzig, Y. Hikida and P.B. Ronne, Higher spin AdS $S_{3}$ supergravity and its dual CFT, JHEP 02 (2012) 109 [arXiv:1111.2139] [INSPIRE].

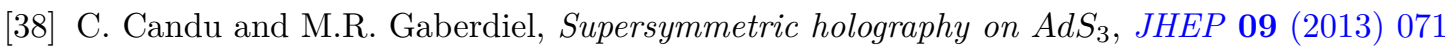
[arXiv: 1203.1939] [INSPIRE].

[39] M. Henneaux, G. Lucena Gómez, J. Park and S.-J. Rey, Super-W(infinity) Asymptotic Symmetry of Higher-Spin AdS $S_{3}$ Supergravity, JHEP 06 (2012) 037 [arXiv:1203.5152] [INSPIRE].

[40] K. Hanaki and C. Peng, Symmetries of Holographic Super-Minimal Models, JHEP 08 (2013) 030 [arXiv: 1203.5768] [INSPIRE].

[41] C. Ahn, The Large N 't Hooft Limit of Kazama-Suzuki Model, JHEP 08 (2012) 047 [arXiv:1206.0054] [INSPIRE].

[42] C. Ahn, The Operator Product Expansion of the Lowest Higher Spin Current at Finite N, JHEP 01 (2013) 041 [arXiv: 1208.0058] [INSPIRE].

[43] A. Castro, A. Faraggi and I. Osorio, A note on the $\mathcal{N}=2$ super- $\mathcal{W}_{3}$ holographic dictionary, JHEP 12 (2020) 177 [arXiv:2008.05541] [INSPIRE].

[44] L.J. Romans, The $N=2$ superW(3) algebra, Nucl. Phys. B 369 (1992) 403 [inSPIRE].

[45] T. Creutzig and Y. Hikida, Rectangular W-algebras, extended higher spin gravity and dual coset CFTs, JHEP 02 (2019) 147 [arXiv:1812.07149] [INSPIRE].

[46] T. Creutzig and Y. Hikida, Rectangular $W$ algebras and superalgebras and their representations, Phys. Rev. D 100 (2019) 086008 [arXiv:1906.05868] [INSPIRE].

[47] T. Creutzig, Y. Hikida and T. Uetoko, Rectangular $W$-algebras of types $\mathrm{SO}(M)$ and $\operatorname{sp}(2 M)$ and dual coset CFTs, JHEP 10 (2019) 023 [arXiv: 1906.05872] [INSPIRE].

[48] Wolfram Research, Inc., Mathematica, Version 12.0, Champaign, IL, U.S.A. (2019).

[49] M.A. Vasiliev, Quantization on sphere and high spin superalgebras, JETP Lett. 50 (1989) 374 [INSPIRE].

[50] M.A. Vasiliev, Higher Spin Algebras and Quantization on the Sphere and Hyperboloid, Int. J. Mod. Phys. A 6 (1991) 1115 [inSPIRE]. 
[51] M.A. Vasiliev, From Coxeter Higher-Spin Theories to Strings and Tensor Models, JHEP 08 (2018) 051 [arXiv : 1804.06520] [INSPIRE].

[52] M. Ammon, P. Kraus and E. Perlmutter, Scalar fields and three-point functions in $D=3$ higher spin gravity, JHEP 07 (2012) 113 [arXiv:1111.3926] [INSPIRE].

[53] T. Creutzig, Y. Hikida and P.B. Ronne, Three point functions in higher spin AdS 3 supergravity, JHEP 01 (2013) 171 [arXiv:1211.2237] [INSPIRE].

[54] C. Ahn and J. Paeng, A supersymmetric enhancement of $\mathcal{N}=1$ holographic minimal model, JHEP 05 (2019) 135 [arXiv:1902.03699] [INSPIRE].

[55] A. Sevrin, W. Troost and A. Van Proeyen, Superconformal Algebras in Two-Dimensions with $N=4$, Phys. Lett. B 208 (1988) 447 [InSPIRE].

[56] A. Sevrin, W. Troost, A. Van Proeyen and P. Spindel, Extended supersymmetric $\sigma$-models on group manifolds (II). Current algebras, Nucl. Phys. B 311 (1988) 465 [InSPIRE].

[57] K. Schoutens, O(n) Extended Superconformal Field Theory in Superspace, Nucl. Phys. B 295 (1988) 634 [INSPIRE].

[58] K. Ferreira, Even spin $\mathcal{N}=4$ holography, JHEP 09 (2017) 110 [arXiv:1702.02641] [INSPIRE].

[59] C. Ahn and H. Kim, Three point functions in the large $\mathcal{N}=4$ holography, JHEP 10 (2015) 111 [arXiv: 1506.00357] [INSPIRE].

[60] M.R. Gaberdiel and R. Gopakumar, Stringy Symmetries and the Higher Spin Square, J. Phys. A 48 (2015) 185402 [arXiv: 1501.07236] [InSPIRE].

[61] M.R. Gaberdiel and R. Gopakumar, String Theory as a Higher Spin Theory, JHEP 09 (2016) 085 [arXiv : 1512.07237] [inSPIRE].

[62] C. Ahn and C. Peng, Chiral Algebras of Two-Dimensional SYK Models, JHEP 07 (2019) 092 [arXiv: 1812.05106] [INSPIRE].

[63] T. Creutzig, Y. Hikida and P.B. Ronne, Higher spin AdS $S_{3}$ holography with extended supersymmetry, JHEP 10 (2014) 163 [arXiv:1406.1521] [INSPIRE].

[64] C. Ahn and H. Kim, Higher spin currents in the enhanced $\mathcal{N}=3$ Kazama-Suzuki model, JHEP 12 (2016) 001 [arXiv:1607.00728] [INSPIRE].

[65] C. Ahn, M.H. Kim and J. Paeng, The operator product expansions in the $\mathcal{N}=4$ orthogonal Wolf space coset model, Eur. Phys. J. C 79 (2019) 578 [arXiv:1904.06855] [INSPIRE].

[66] C. Ahn and J. Paeng, Higher Spin Currents in Orthogonal Wolf Space, Class. Quant. Grav. 32 (2015) 045011 [arXiv: 1410.0080] [INSPIRE].

[67] F.W.J. Olver et al. eds., NIST Digital Library of Mathematical Functions, Release 1.0.27 of 2020-06-15 http://dlmf.nist.gov/. 